\title{
NECKPINCH DYNAMICS FOR ASYMMETRIC SURFACES EVOLVING BY MEAN CURVATURE FLOW
}

\author{
ZHOU GANG, DAN KNOPF, AND ISRAEL MICHAEL SIGAL
}

\begin{abstract}
We study noncompact surfaces evolving by mean curvature flow $(\mathrm{MCF})$. For an open set of initial data that are $C^{3}$-close to round, but without assuming rotational symmetry or positive mean curvature, we show that MCF solutions become singular in finite time by forming neckpinches, and we obtain detailed asymptotics of that singularity formation. Our results show in a precise way that MCF solutions become asymptotically rotationally symmetric near a neckpinch singularity.
\end{abstract}

\section{CONTENTS}

1. Introduction

2. Basic evolution equations

3. Implied evolution equations

4. The first bootstrap machine

4.1. Input

4.2. Output

4.3. Structure

6. Decay estimates in the inner region $\quad 15$

6.1. Differential inequalities 15

6.2. Lyapunov functionals of second and third order 19

6.3. Lyapunov functionals of fourth and fifth order 24

6.4. Estimates of second- and third-order derivatives 25

7. Estimates in the outer region 25

7.1. Second-order decay estimates $\quad 25$

7.2. Third-order decay estimates $\quad 26$

7.3. Third-order smallness estimates 28

8. The second bootstrap machine 30

8.1. Input 31

8.2. Output 32

8.3. Structure 32

9. Evolution equations for the decomposition 33

10. Estimates to control the parameters $a$ and $b \quad 35$

11. Estimates to control the fluctuation $\phi \quad 38$

11.1. Proof of estimate (9.12) 43

11.2. Proof of estimate (9.13) 48

11.3. Proof of estimate (9.15) 51

ZG thanks NSF for supports in DMS-1308985. DK thanks NSF for support in DMS-0545984. IMS thanks NSERC for support in NA7901. 
11.4. Proof of estimate (9.14) 54

12. Proof of the Main Theorem 55

Appendix A. Mean curvature flow of normal graphs $\quad 56$

Appendix B. Interpolation estimates 58

Appendix C. A parabolic maximum principle for noncompact domains $\quad 59$

Appendix D. Estimates of higher-order derivatives 60

$\begin{array}{ll}\text { References } & 67\end{array}$

\section{INTRODUCTION}

In this paper, we study the motion of cylindrical surfaces under mean curvature flow (MCF). Specifically, we investigate the asymptotics of finite-time singularity formation for such surfaces, without assuming rotational symmetry or positive mean curvature.

There is a rich literature on MCF singularity formation, one far too vast to be fully acknowledged here. Neckpinch singularities for rotationally symmetric 2dimensional surfaces were first observed by Huisken [10]; this was generalized to higher dimensions by Simon [12]. Asymptotic properties of a solution approaching a singularity were studied by Angenent-Velázquez [3], who obtained rigorous asymptotics for nongeneric Type-II "degenerate neckpinches," and derived formal matched asymptotics for (conjecturally generic) Type-I singularities. Rigorous asymptotics for the Type-I case were recently obtained by two of the authors [9]. The corresponding result for Type-I Ricci flow neckpinches was obtained by Angenent and another of the authors [2]. For the Type-II Ricci flow case, see [1]. ${ }^{1}$ These asymptotic analyses all use the hypothesis of rotational symmetry in essential ways.

A different approach is the surgery program of Huisken and Sinestrari [11], which shows that singularities of 2-convex immersions $\mathcal{M}^{n} \subset \mathbb{R}^{n+1}$ with $n \geq 3$ are either close to round $\mathbb{S}^{n}$ components or close to "necklike" $\mathbb{S}^{n-1} \times I$ components; this program does not involve asymptotics or require symmetry hypothesis. Yet another approach studies weak solutions that extend past the singularity time. See existence results of Brakke [4], Evans-Spruck [8], and Chen-Giga-Goto [5], as well as recent classification results by Colding-Minicozzi [6].

In this paper, we consider the evolution of non-symmetric, noncompact surfaces embedded in $\mathbb{R}^{3}$. To analyze their singularity formation, we study rescaled solutions defined with respect to adaptive blowup variables. We remove the hypothesis of rotational symmetry and instead consider initial data in a Sobolev space $H^{5}\left(\mathbb{S}^{1} \times \mathbb{R} ; \mathrm{d} \theta \sigma \mathrm{d} y\right.$ ) (where the weighted measure $\sigma \sim|y|^{-\frac{6}{5}}$ as $|y| \rightarrow \infty$ ) which are $C^{3}$-close to round necks and possess weaker symmetries. (These symmetries are retained to reduce technicalities but are not essential to our approach.) The assumptions are made precise in Section 2. Because we do not allow fully asymmetric initial data, our results do not establish uniqueness of the limiting cylinder for general MCF neckpinch singularities (a conjecture that we learned of from Klaus Ecker). However, our results do provide rigorous evidence in favor of the heuristic expectation that rotational symmetry is stable in a suitable (quasi-isometric) sense. In light of the result [6] of Colding-Minicozzi that shrinking spheres and

\footnotetext{
${ }^{1}$ Note that what we call the "inner region" in this paper is called the "intermediate region" in [1] and [2].
} 
cylinders are the only generic MCF singularity models, our work suggests that the rotationally-symmetric neckpinch is in fact a "universal" singularity profile.

Our analysis consists of two largely independent parts, both of which are organized as bootstrap machines. The first machine takes as its input certain (weak) estimates that follow from our assumptions on the initial data; by parabolic regularization for quasilinear equations, we may assume that these hold for a sufficiently short time interval. These estimates are detailed in Section 4. The output of the machine consists of improved a priori estimates for the same time interval, which may then be propagated forward in time. The machine is constructed in Sections 4-7. Its construction employs Lyapunov functionals and Sobolev embedding arguments near the center of the neck, where the most critical analysis is needed, together with maximum-principle arguments away from the developing singularity.

The second bootstrap machine takes for its input similar (weak) conditions detailed in Section 8, along with the improved a priori estimates output by the first machine. It further improves those estimates by showing that for correct choices of adaptive scaling parameters, it is possible to decompose a solution into an asymptotically dominant profile and a far smaller "remainder" term. The methods employed here are close to those used in [9], and are collected in Sections 8-11.

Connecting the two machines yields a (non-circular) sequence of arguments that establishes finite-time extinction of the unrescaled solution, its singular collapse, the nature of the singular set, and the solution's asymptotic behavior in a spacetime neighborhood of the singularity. Thus when combined, these two bootstrap arguments imply our main theorem. We prove this in Section 12.

As we recall in Section 2, MCF of a normal graph over a 2-dimensional cylinder is determined up to tangential diffeomorphisms by a radius function $u(x, \theta, t)>0$. The assumptions of the theorem are satisfied by any smooth reflection-symmetric and $\pi$-periodic initial surface $u_{0}(x, \theta)=u(x, \theta, 0)$ that is a sufficiently small perturbation of the surfaces studied in [9].

Main Theorem. Let $u(x, \theta, t)$ be a solution of MCF whose initial surface satisfies the Main Assumptions stated in Section 2 for sufficiently small $0<b_{0}, c_{0} \ll 1$. Then there exists a time $T<\infty$ such that $u(x, \theta, t)$ develops a neckpinch singularity at time $T$, with $u(0, \cdot, T)=0$. There exist functions $\lambda(t), b(t), c(t)$, and $\phi(x, \theta, t)$ such that with $y(x, t):=\lambda(t)^{-1} x$, one has

$$
\frac{u(x, \theta, t)}{\lambda(t)}=\sqrt{\frac{2+b(t) y^{2}}{c(t)}}+\phi(y, \theta, t),
$$

where as $t \nearrow T$,

$$
\begin{aligned}
\lambda(t) & =[1+o(1)] \lambda_{0} \sqrt{T-t}, \\
b(t) & =\left[1+\mathcal{O}\left((-\log (T-t))^{-\frac{1}{2}}\right)\right](-\log (T-t))^{-1}, \\
c(t) & =1+\left[1+\mathcal{O}\left((-\log (T-t))^{-1}\right)\right](-\log (T-t))^{-1} .
\end{aligned}
$$

The solution is asymptotically rotationally symmetric near the neckpinch singularity in the precise sense that, with $v(y, \theta, \tau):=\lambda(t)^{-1} u(x, \theta, t)$, the estimates

$v^{-2}\left|\partial_{\theta} \phi\right|+v^{-1}\left|\partial_{y} \partial_{\theta} \phi\right|+v^{-2}\left|\partial_{\theta}^{2} \phi\right|+v^{-1}\left|\partial_{y}^{2} \partial_{\theta} \phi\right|+v^{-2}\left|\partial_{y} \partial_{\theta}^{2} \phi\right|+v^{-3}\left|\partial_{\theta}^{3} \phi\right|=\mathcal{O}\left(b(t)^{\frac{33}{20}}\right)$ 
and

$$
\frac{|\phi(y, \theta, t)|}{\left(1+y^{2}\right)^{\frac{3}{2}}}=\mathcal{O}\left(b(t)^{\frac{8}{5}}\right) \quad \text { and } \quad \frac{|\phi(y, \theta, t)|}{\left(1+y^{2}\right)^{\frac{11}{20}}}=\mathcal{O}\left(b(t)^{\frac{13}{20}}\right)
$$

all hold uniformly as $t \nearrow T$.

\section{Basic eVolution equations}

In this paper, we study the evolution of graphs over a cylinder $\mathbb{S}^{1} \times \mathbb{R}$ embedded in $\mathbb{R}^{3}$. In coordinates $(x, y, z)$ for $\mathbb{R}^{3}$, we take as an initial datum a surface $M_{0}$ around the $x$-axis, given by a map $\sqrt{y^{2}+z^{2}}=u_{0}(x, \theta)$, where $\theta$ denotes the angle from the ray $y>0$ in the $(y, z)$-plane. Then for as long as the flow remains a graph, all $M_{t}$ are given by $\sqrt{y^{2}+z^{2}}=u(x, \theta, t)$. It follows from equations (A.3) and (A.4), derived in Appendix A, that $u$ evolves by

$$
\partial_{t} u=\frac{\left[1+\left(\frac{\partial_{\theta} u}{u}\right)^{2}\right] \partial_{x}^{2} u+\frac{1+\left(\partial_{x} u\right)^{2}}{u^{2}} \partial_{\theta}^{2} u-2 \frac{\left(\partial_{x} u\right)\left(\partial_{\theta} u\right)^{2}}{u^{3}} \partial_{x} \partial_{\theta} u-\frac{\left(\partial_{\theta} u\right)^{2}}{u^{3}}}{1+\left(\partial_{x} u\right)^{2}+\left(\frac{\partial_{\theta} u}{u}\right)^{2}}-\frac{1}{u}
$$

with initial condition $u(x, \theta, 0)=u_{0}(x, \theta)$.

Analysis of rotationally symmetric neckpinch formation [9] leads one to expect that solutions of (A.3) will become singular in finite time, resembling spatially homogeneous ODE solutions $\sqrt{2(T-t)}$ in an suitable space-time neighborhood of the developing singularity.

Accordingly, we apply adaptive rescaling, transforming the original space-time variables $x$ and $t$ into rescaled blowup variables

$$
y(x, t):=\lambda^{-1}(t)\left[x-x_{0}(t)\right]
$$

and

$$
\tau(t):=\int_{0}^{t} \lambda^{-2}(s) \mathrm{d} s
$$

respectively, where $x_{0}(t)$ marks the center of the neck. What distinguishes this approach from standard parabolic rescaling (see, e.g., [3] or [2]) is that we do not fix $\lambda(t)$ but instead consider it as a free parameter to be determined from the evolution of $u$ in equation (2.1). ${ }^{2}$ By [9], one expects that $\lambda \approx \sqrt{T-t}$ and $\tau \approx-\log (T-t)$, where $T>0$ is the singularity time. (We confirm this in Section 12 below.)

Consider a solution $u(x, \theta, t)$ of $(2.1)$ with initial condition $u_{0}(x, \theta)$. We define a rescaled radius $v(y, \theta, \tau)$ by

$$
v(y(x, t), \theta, \tau(t)):=\lambda^{-1}(t) u(x, \theta, t) .
$$

Then $v$ initially satisfies $v(y, \theta, 0)=v_{0}(y, \theta)$, where $v_{0}(y, \theta):=\lambda_{0}^{-1} u_{0}\left(\lambda_{0} y, \theta\right)$, with $\lambda_{0}$ the initial value of the scaling parameter $\lambda$.

In commuting $(y, \theta, \tau)$ variables, the quantity $v$ evolves by

$$
\partial_{\tau} v=A_{v} v+a v-v^{-1},
$$

where $A_{v}$ is the quasilinear elliptic operator

$$
A_{v}:=F_{1}(p, q) \partial_{y}^{2}+v^{-2} F_{2}(p, q) \partial_{\theta}^{2}+v^{-1} F_{3}(p, q) \partial_{y} \partial_{\theta}+v^{-2} F_{4}(p, q) \partial_{\theta}-a y \partial_{y}
$$

with $v$-dependent coefficients defined with respect to

$$
a:=-\lambda \partial_{t} \lambda, \quad p:=\partial_{y} v, \quad \text { and } \quad q:=v^{-1} \partial_{\theta} v,
$$

\footnotetext{
${ }^{2}$ For fully general neckpinches, one would also regard $x_{0}(t)$ as a free parameter; below, we impose reflection symmetry to fix $x_{0}(t) \equiv 0$.
} 
by

$$
\begin{array}{lll}
F_{1}(p, q):=\frac{1+q^{2}}{1+p^{2}+q^{2}}, & F_{2}(p, q):=\frac{1+p^{2}}{1+p^{2}+q^{2}} \\
F_{3}(p, q):=-\frac{2 p q}{1+p^{2}+q^{2}}, & F_{4}(p, q):=\frac{q}{1+p^{2}+q^{2}} .
\end{array}
$$

In order to state our assumptions precisely, we introduce some further notation. We denote the formal solution of the adiabatic approximation to equation (2.5) by

$$
V_{r, s}(y):=\sqrt{\frac{2+s y^{2}}{\frac{1}{2}+r}},
$$

where $r$ and $s$ are positive parameters. We introduce a step function ${ }^{3}$

$$
g(y, s):=\left\{\begin{array}{ccc}
\frac{9}{10} \sqrt{2} & \text { if } \quad s y^{2}<20 \\
4 & \text { if } \quad s y^{2} \geq 20
\end{array}\right.
$$

We define a norm $\|\cdot\|_{m, n}$ by

$$
\|\phi\|_{m, n}:=\left\|\left(1+y^{2}\right)^{-\frac{m}{2}} \partial_{y}^{n} \phi\right\|_{L^{\infty}} .
$$

We also introduce a Hilbert space $L_{\sigma}^{2} \equiv L^{2}\left(\mathbb{S}^{1} \times \mathbb{R} ; \mathrm{d} \theta \sigma \mathrm{d} y\right)$ with norm $\|\cdot\|_{\sigma}$, whose weighted measure is defined with respect to $\Sigma \gg 1$ (to be fixed below) by

$$
\sigma(y):=\left(\Sigma+y^{2}\right)^{-\frac{3}{5}} .
$$

Here are our assumptions. We state them for $v_{0}(y, \theta)=v(y, \theta, 0)$, but they easily translate to $u_{0}(x, \theta)=u(x, \theta, 0)$ using the relation $u_{0}(x, \theta)=\lambda_{0} v\left(\lambda_{0}^{-1} x, \theta\right)$.

Main Assumptions. There exist small positive constants $b_{0}, c_{0}$ such that:

[A1] The initial surface is a graph over $\mathbb{S}^{1} \times \mathbb{R}$ determined by a smooth function $v_{0}(y, \theta)>0$ with the symmetries $v_{0}(y, \theta)=v_{0}(-y, \theta)=v_{0}(y, \theta+\pi)$.

[A2] The initial surface satisfies $v_{0}(y, \cdot)>g\left(y, b_{0}\right)$.

[A3] The initial surface is a small deformation of a formal solution $V_{a_{0}, b_{0}}$ in the sense that for $(m, n) \in\{(3,0),(11 / 10,0),(2,1),(1,1)\}$, one has

$$
\left\|v_{0}-V_{a_{0}, b_{0}}\right\|_{m, n}<b_{0}^{\frac{m+n}{2}+\frac{1}{10}} .
$$

[A4] The parameter $a_{0}=a(0)$ obeys the bound $\left|a_{0}-1 / 2\right|<c_{0}$.

[A5] The initial surface obeys the further derivative bounds:

$$
\begin{aligned}
& \sum_{n \neq 0,2 \leq m+n \leq 3} v_{0}^{-n}\left|\partial_{y}^{m} \partial_{\theta}^{n} v_{0}\right|<b_{0}^{2}, \\
& b_{0} v_{0}^{-\frac{1}{2}}\left|\partial_{y} v_{0}\right|+b_{0}^{\frac{1}{2}}\left|\partial_{y}^{2} v_{0}\right|+\left|\partial_{y}^{3} v_{0}\right|<b_{0}^{\frac{3}{2}}, \\
&\left|\partial_{y} \partial_{\theta}^{2} v_{0}\right|+v_{0}^{-1}\left|\partial_{\theta}^{3} v_{0}\right|<c_{0} .
\end{aligned}
$$

[A6] $b_{0}^{\frac{4}{5}}\left\|\partial_{y}^{4} v_{0}\right\|_{\sigma}+\left\|\partial_{y}^{5} v_{0}\right\|_{\sigma}+\sum_{n \neq 0,4 \leq m+n \leq 5}\left\|v_{0}^{-n} \partial_{y}^{m} \partial_{\theta}^{n} v_{0}\right\|_{\sigma}<b_{0}^{4}$.

Our method works because MCF preserves the symmetries in Assumption [A1].

\footnotetext{
${ }^{3}$ One motivation for this choice of $g$ may be found in the results of [9], which prove that $v=(1+o(1)) \sqrt{2+b(\tau) y^{2}}$ as $\tau \rightarrow \infty$ for the rotationally symmetric MCF neckpinch. For another motivation, see Remark 3.2 below.
} 
Lemma 2.1. If $v_{0}$ has the symmetries $v_{0}(y, \theta)=v_{0}(-y, \theta)=v_{0}(y, \theta+\pi)$, then the solution $v$ of equation (2.5) shares the same symmetries, $v(y, \theta, \tau)=v(-y, \theta, \tau)=$ $v(y, \theta+\pi, \tau)$, for as long as it remains a graph.

Proof. If $v(y, \theta, \tau)$ solves equation (2.5), so do $v(-y, \theta, \tau)$ and $v(y, \theta+\pi, \tau)$. Thus the result follows from local well-posedness of the equation.

\section{IMPLIED EVOLUTION EQUATIONS}

Recall from equation (2.5) in Section 2 that $\partial_{\tau} v=A_{v} v+a v-v^{-1}$, where $A_{v}$ is the quasilinear operator defined in equation (2.6), with coefficients $F_{\ell}$ introduced

in definitions (2.7)-(2.8). Our goal in this section is to derive evolution equations for quantities of the form

$$
v_{m, n, k}:=v^{-k}\left(\partial_{y}^{m} \partial_{\theta}^{n} v\right),
$$

defined with respect to integers $m, n \geq 0$ and a real number $k \geq 0$.

Lemma 3.1. The quantity $v_{m, n, k}$ evolves by

$$
\partial_{\tau} v_{m, n, k}=\left\{A_{v}+(k+1) v^{-2}-(m+k-1) a\right\} v_{m, n, k}+E_{m, n, k},
$$

where the individual terms of the nonlinear quantity $E_{m, n, k}=\sum_{\ell=0}^{5} E_{m, n, k, \ell}$ are defined by

$$
\begin{aligned}
& E_{m, n, k, 0}:=-k v^{-1} v_{m, n, k}\left(A_{v} v+a y \partial_{y} v\right) \\
& E_{m, n, k, 1}:=v^{-k} \partial_{y}^{m} \partial_{\theta}^{n}\left(F_{1} \partial_{y}^{2} v\right)-F_{1} \partial_{y}^{2} v_{m, n, k} \\
& E_{m, n, k, 2}:=v^{-k} \partial_{y}^{m} \partial_{\theta}^{n}\left(v^{-2} F_{2} \partial_{\theta}^{2} v\right)-v^{-2} F_{2} \partial_{\theta}^{2} v_{m, n, k}, \\
& E_{m, n, k, 3}:=v^{-k} \partial_{y}^{m} \partial_{\theta}^{n}\left(v^{-1} F_{3} \partial_{y} \partial_{\theta} v\right)-v^{-1} F_{3} \partial_{y} \partial_{\theta} v_{m, n, k} \\
& E_{m, n, k, 4}:=v^{-k} \partial_{y}^{m} \partial_{\theta}^{n}\left(v^{-2} F_{4} \partial_{\theta} v\right)-v^{-2} F_{4} \partial_{\theta} v_{m, n, k}, \\
& E_{m, n, k, 5}:=-v^{-k} \partial_{y}^{m} \partial_{\theta}^{n}\left(v^{-1}\right)-v^{-2} v_{m, n, k}
\end{aligned}
$$

Proof. We begin by using equations (2.5) and (2.6) to compute that

$$
\begin{aligned}
\partial_{\tau}\left(v^{-k} \partial_{y}^{m} \partial_{\theta}^{n} v\right)= & -k v_{m, n, k} v^{-1} \partial_{\tau} v+v^{-k} \partial_{y}^{m} \partial_{\theta}^{n}\left(\partial_{\tau} v\right) \\
= & -k v^{-1} v_{m, n, k}\left(A_{v} v\right)-k a v_{m, n, k}+k v^{-2} v_{m, n, k} \\
& +v^{-k} \partial_{y}^{m} \partial_{\theta}^{n}\left\{F_{1} \partial_{y}^{2} v+v^{-2} F_{2} \partial_{\theta}^{2} v+v^{-1} F_{3} \partial_{y} \partial_{\theta} v+v^{-2} F_{4} \partial_{\theta} v\right\} \\
& -a v^{-k} \partial_{y}^{m} \partial_{\theta}^{n}\left(y \partial_{y} v\right)+a v_{m, n, k}-v^{-k} \partial_{y}^{m} \partial_{\theta}^{n}\left(v^{-1}\right) .
\end{aligned}
$$


We then rewrite this in the form

$$
\begin{aligned}
\partial_{\tau} v_{m, n, k}= & A_{v} v_{m, n, k}+\left\{(k+1) v^{-2}+a(1-k)\right\} v_{m, n, k} \\
& -k v^{-1} v_{m, n, k}\left(A_{v} v\right) \\
& +v^{-k} \partial_{y}^{m} \partial_{\theta}^{n}\left(F_{1} \partial_{y}^{2} v\right)-F_{1} \partial_{y}^{2} v_{m, n, k} \\
& +v^{-k} \partial_{y}^{m} \partial_{\theta}^{n}\left(v^{-2} F_{2} \partial_{\theta}^{2} v\right)-v^{-2} F_{2} \partial_{\theta}^{2} v_{m, n, k} \\
& +v^{-k} \partial_{y}^{m} \partial_{\theta}^{n}\left(v^{-1} F_{3} \partial_{y} \partial_{\theta} v\right)-v^{-1} F_{3} \partial_{y} \partial_{\theta} v_{m, n, k} \\
& +v^{-k} \partial_{y}^{m} \partial_{\theta}^{n}\left(v^{-2} F_{4} \partial_{\theta} v\right)-v^{-2} F_{4} \partial_{\theta} v_{m, n, k} \\
& -v^{-k} \partial_{y}^{m} \partial_{\theta}^{n}\left(v^{-1}\right)-v^{-2} v_{m, n, k} \\
& +a\left\{y \partial_{y} v_{m, n, k}-v^{-k} \partial_{y}^{m} \partial_{\theta}^{n}\left(y \partial_{y} v\right)\right\} .
\end{aligned}
$$

Comparing this to equations (3.2) and (3.3) shows that to complete the proof, it suffices to observe that the final line above simplifies as follows:

$$
a\left\{y \partial_{y} v_{m, n, k}-v^{-k} \partial_{y}^{m} \partial_{\theta}^{n}\left(y \partial_{y} v\right)\right\}=-m a v_{m, n, k}-k v^{-1} v_{m, n, k}\left(a y \partial_{y} v\right) .
$$

Remark 3.2. An important feature of the calculation above is its linear reaction term, which reveals how the evolution equation for $v_{m, n, k}$ "improves" as $m$ and $k$ increase, provided that $v$ is suitably bounded from below. This partly explains our choice of step function $g$ in equation (2.10).

Corollary 3.3. The quantity $v_{m, n, k}^{2}$ evolves by

$$
\begin{aligned}
\partial_{\tau} v_{m, n, k}^{2}= & A_{v}\left(v_{m, n, k}^{2}\right)+2\left[(k+1) v^{-2}-(m+k-1) a\right] v_{m, n, k}^{2} \\
& -B_{m, n, k}+2 E_{m, n, k} v_{m, n, k},
\end{aligned}
$$

where $B_{m, n, k}:=A_{v}\left(v_{m, n, k}^{2}\right)-2 v_{m, n, k} A_{v} v_{m, n, k}$ satisfies $B \geq 0$ along with

$$
B_{m, n, k} \geq \frac{v_{m+1, n, k}^{2}+v_{m, n+1, k+1}^{2}}{1+p^{2}+q^{2}}-k^{2}\left(v_{1,0,1}^{2}+v_{0,1,1}^{2}\right) v_{m, n, k}^{2}
$$

Proof. Recall the useful fact that $v \partial_{y}^{2} v=\frac{1}{2} \partial_{y}^{2}\left(v^{2}\right)-\left(\partial_{y} v\right)^{2}$. Using this idea, the result follows by applying Cauchy-Schwarz to obtain first

$$
\begin{aligned}
B_{m, n, k} & =2\left[F_{1}\left(\partial_{y} v_{m, n, k}\right)^{2}+v^{-2} F_{2}\left(\partial_{\theta} v_{m, n, k}\right)^{2}+v^{-1} F_{3}\left(\partial_{y} v_{m, n, k}\right)\left(\partial_{\theta} v_{m, n, k}\right)\right] \\
& \geq 2 \frac{\left(\partial_{y} v_{m, n, k}\right)^{2}+\left(\partial_{\theta} v_{m, n, k}\right)^{2}}{1+p^{2}+q^{2}} \geq 0,
\end{aligned}
$$

and then applying it in case $k>0$ to the identities

$$
\begin{aligned}
& \left(\partial_{y} v_{m, n, k}\right)^{2}=v_{m+1, n, k}^{2}-2 v_{m+1, n, k} k v_{1,0,1} v_{m, n, k}+k^{2} v_{1,0,1}^{2} v_{m, n, k}^{2}, \\
& \left(\partial_{\theta} v_{m, n, k}\right)^{2}=v_{m, n+1, k}^{2}-2 v_{m, n+1, k} k v_{0,1,1} v_{m, n, k}+k^{2} v_{0,1,1}^{2} v_{m, n, k}^{2} .
\end{aligned}
$$

Remark 3.4. Once one has suitable first-order estimates for $v$, one can bound the quantity $1+p^{2}+q^{2}$ from above, whereupon $B_{m, n, k}$ contributes valuable higher-order terms of the form $-\varepsilon\left(v_{m+1, n, k}^{2}+v_{m, n+1, k+1}^{2}\right)$ to the evolution equation satisfied by $v_{m, n, k}$. The same first-order estimates let us easily control the potentially bad terms in $(3.5)$ by $k^{2}\left(v_{1,0,1}^{2}+v_{0,1,1}^{2}\right) v_{m, n, k}^{2} \leq \varepsilon v_{m, n, k}^{2}$. 
In estimating the "commutators" $E_{m, n, k, \ell}$ defined in (3.3), the following simple observation will be used many times, often implicitly. We omit its easy proof.

Lemma 3.5. For any $i, j \geq 0$ and $\ell=1, \ldots, 4$, there exist constants $C_{i, j, \ell}$ such that

for all $p, q \in \mathbb{R}$.

$$
\left|\partial_{p}^{i} \partial_{q}^{j} F_{\ell}(p, q)\right| \leq C_{i, j, \ell}
$$

We also freely use the following facts, usually without comment.

Remark 3.6. The quantity $E_{m, n, k, 0}$ vanishes if $k=0$.

Remark 3.7. The quantity $E_{m, n, k, 5}$ vanishes if $m+n=1$.

\section{THE FIRST BOOTSTRAP MACHINE}

4.1. Input. Here are the conditions that constitute the input to our first bootstrap machine, whose structure we describe below. By standard regularity theory for quasilinear parabolic equations, if the initial data satisfy the Main Assumptions in Section 2 for $b_{0}$ and $c_{0}$ sufficiently small, then the solution will satisfy the properties below up to a short time $\tau_{1}>0$. (Note that to obtain some of the derivative bounds below, one uses the general interpolation result, Lemma B.2.)

Some of these properties are global, while others are local in nature. In these conditions, and in many of the arguments that follow, we separately treat the inner region $\left\{\beta y^{2} \leq 20\right\}$ and the outer region $\left\{\beta y^{2} \geq 20\right\}$, both defined with respect to

$$
\beta(\tau):=(\kappa+\tau)^{-1},
$$

where $\kappa=\kappa\left(b_{0}, c_{0}\right) \gg 1$. Note that our Main Assumptions imply that slightly stronger conditions hold for the inner region. This is unsurprising: it is natural to expect that the solution of equation (2.5) is sufficiently close in that region to the solution $V_{\frac{1}{2}, \beta}$ of the equation $\frac{1}{2} y \partial_{y} V-\frac{1}{2} V+V^{-1}=0$ that is an adiabatic approximation of (2.5) there. (Compare [2] and [9].)

Here are the global conditions: ${ }^{4}$

[C0] For $\tau \in\left[0, \tau_{1}\right]$, the solution has the uniform lower bound $v(\cdot, \cdot, \tau) \geq \kappa^{-1}$.

$[\mathrm{C} 1]$ For $\tau \in\left[0, \tau_{1}\right]$, the solution satisfies the first-order estimates

$$
\left|\partial_{y} v\right| \lesssim \beta^{\frac{2}{5}} v^{\frac{1}{2}}, \quad\left|\partial_{\theta} v\right| \lesssim \beta^{\frac{3}{2}} v^{2}, \quad \text { and } \quad\left|\partial_{\theta} v\right| \lesssim v .
$$

[C2] For $\tau \in\left[0, \tau_{1}\right]$, the solution satisfies the second-order estimates

$$
\left|\partial_{y}^{2} v\right| \lesssim \beta^{\frac{3}{5}}, \quad\left|\partial_{y} \partial_{\theta} v\right| \lesssim \beta^{\frac{3}{2}} v, \quad\left|\partial_{y} \partial_{\theta} v\right| \lesssim 1, \quad \text { and } \quad\left|\partial_{\theta}^{2} v\right| \lesssim \beta^{\frac{3}{2}} v^{2} .
$$

[C3] For $\tau \in\left[0, \tau_{1}\right]$ the solution satisfies the third-order decay estimates

$$
\left|\partial_{y}^{3} v\right| \lesssim \beta \text { and } v^{-n}\left|\partial_{y}^{m} \partial_{\theta}^{n} v\right| \lesssim \beta^{\frac{3}{2}}
$$

for $m+n=3$ with $n \geq 1$, as well as the "smallness estimate" that

$$
\beta^{-\frac{11}{20}}\left(\left|\partial_{y}^{3} v\right|+\left|\partial_{y}^{2} \partial_{\theta} v\right|\right)+\left|\partial_{y} \partial_{\theta}^{2} v\right|+v^{-1}\left|\partial_{\theta}^{3} v\right| \lesssim\left(\beta_{0}+\varepsilon_{0}\right)^{\frac{1}{40}}
$$

for some $\varepsilon_{0}=\varepsilon_{0}\left(b_{0}, c_{0}\right) \ll 1 .^{5}$

\footnotetext{
${ }^{4}$ Notation: In the remainder of this paper, we write $\varphi \lesssim \psi$ if there exists a uniform constant $C>0$ such that $\varphi \leq C \psi$, and we define $\langle x\rangle:=\sqrt{1+|x|^{2}}$.

${ }^{5}$ The smallness estimate will only be used in the proof of Theorem 4.7.
} 
[Ca] For $\tau \in\left[0, \tau_{1}\right]$, the parameter $a$ satisfies $^{6}$

$$
\frac{1}{2}-\kappa^{-1} \leq a \leq \frac{1}{2}+\kappa^{-1}
$$

To state the remaining global conditions, we decompose the solution into $\theta$ independent and $\theta$-dependent parts $v_{1}, v_{2}$, respectively, defined by

$$
v_{1}(y, \tau):=\frac{1}{2 \pi} \int_{0}^{2 \pi} v(y, \theta, \tau) \mathrm{d} \theta \quad \text { and } \quad v_{2}(y, \theta, \tau):=v(y, \theta, \tau)-v_{1}(y, \tau)
$$

We denote the norm in the Hilbert space $L_{\sigma}^{2}$ introduced in definition (2.11) by $\|\cdot\|_{\sigma}$, and the inner product by

$$
\langle\varphi, \psi\rangle_{\sigma}:=\int_{\mathbb{R}} \int_{\mathbb{S}^{1}} \varphi \psi \mathrm{d} \theta \sigma \mathrm{d} y
$$

Our remaining inputs are:

[Cs] For $\tau \in\left[0, \tau_{1}\right]$, one has Sobolev bounds $\left\|v^{-n} \partial_{y}^{m} \partial_{\theta}^{n} v\right\|_{\sigma}<\infty$ whenever $4 \leq m+n \leq 7$.

[Cr] There exists $0<\delta \ll 1$ such that the scale-invariant bound $\left|v_{2}\right| \leq \delta v_{1}$ holds everywhere for $\tau \in\left[0, \tau_{1}\right]$.

[Cg] For $\tau \in\left[0, \tau_{1}\right]$, one has $\langle y\rangle^{-1}\left|\partial_{y} v\right| \leq \Sigma^{\frac{1}{4}} \beta$.

Remark 4.1. Note that our global gradient Condition [Cg] posits the $\beta$ decay rate of the formal solution $V(y, \tau)=\sqrt{2+\beta y^{2}}$ but with a large constant $\Sigma^{\frac{1}{4}} \gg 1$. The effect of our bootstrap argument will be to sharpen that constant.

Remark 8.1 (below) shows that Condition [Cb] in Section 8, which is directly implied by our Main Assumptions, in turn implies that $[\mathrm{Cg}]$ holds, along with extra properties that are local to the inner region:

[C0i] For $\tau \in\left[0, \tau_{1}\right]$ and $\beta y^{2} \leq 20$, the quantity $v$ is uniformly bounded from above and below, so that for $\beta y^{2} \leq 20$, one has bounds

$$
\frac{9}{10} \sqrt{2} \equiv g(y, \beta) \leq v(y, \cdot, \cdot) \leq C_{0}
$$

[C1i] For $\tau \in\left[0, \tau_{1}\right]$ and $\beta y^{2} \leq 20$, the solution satisfies the stronger first-order estimates

$$
\left|\partial_{y} v\right| \lesssim \beta^{\frac{1}{2}} v^{\frac{1}{2}} \text { and }\left|\partial_{\theta} v\right| \lesssim \kappa^{-\frac{1}{2}} v
$$

\footnotetext{
${ }^{6}$ Proposition 8.3 will establish that $a$ is a $C^{1}$ function of time.
} 
4.2. Output. The output of this machine consists of the following estimates, which collectively improve Conditions [C0]-[C3], [Cs], [Cr], [Cg], and [C0i]-[C1i]:

$$
\begin{gathered}
v(y, \theta, \tau) \geq g(y, \beta), \quad v=\mathcal{O}(\langle y\rangle) \text { as }|y| \rightarrow \infty \\
v^{-\frac{1}{2}}\left|\partial_{y} v\right| \lesssim \beta^{\frac{1}{2}}, \quad\left|\partial_{y} v\right| \lesssim 1, \quad v^{-2}\left|\partial_{\theta} v\right| \lesssim \beta^{\frac{33}{20}}, \quad v^{-1}\left|\partial_{\theta} v\right| \lesssim \kappa^{-\frac{1}{2}} \\
\beta\left|\partial_{y}^{2} v\right|+v^{-1}\left|\partial_{y} \partial_{\theta} v\right|+v^{-2}\left|\partial_{\theta}^{2} v\right| \lesssim \beta^{\frac{33}{20}} \\
\left|\partial_{y} \partial_{\theta} v\right|+v^{-1}\left|\partial_{\theta}^{2} v\right| \lesssim\left(\beta_{0}+\varepsilon_{0}\right)^{\frac{1}{20}} \\
\beta^{\frac{1}{2}}\left|\partial_{y}^{3} v\right|+v^{-1}\left|\partial_{y}^{2} \partial_{\theta} v\right|+v^{-2}\left|\partial_{y} \partial_{\theta}^{2} v\right|+v^{-3}\left|\partial_{\theta}^{3} v\right| \lesssim \beta^{\frac{33}{20}} \\
\beta^{-\frac{11}{20}}\left(\left|\partial_{y}^{3} v\right|+\left|\partial_{y}^{2} \partial_{\theta} v\right|\right)+\left|\partial_{y} \partial_{\theta}^{2} v\right|+v^{-1}\left|\partial_{\theta}^{3} v\right| \lesssim\left(\beta_{0}+\varepsilon_{0}\right)^{\frac{1}{20}}
\end{gathered}
$$

Here, $\beta_{0} \equiv \beta(0)$ and $\varepsilon_{0}$ are independent of $\tau_{1}$. So these improvements will allow us to propagate the assumptions above forward in time. (See Section 12 below.)

4.3. Structure. Here is how we establish these stronger estimates.

We first derive improved estimates for $v$ and its first derivatives in the outer region, using our Main Assumptions and their implications [Ca] and [C0]-[C2] for $v$ and its first and second derivatives, and using the stronger estimates [C0i]-[C1i] that hold on the boundary circles $\beta y^{2}=20$. The reason why second-order conditions are needed to prove improved first-order estimates is that the nonlinear terms in the evolution equation for $D^{N} v$ (a spatial derivative of total order $N$ ) will in general contain derivatives of order $N+1$. This occurs because first derivatives of $v$ appear in the quasilinear operator $A_{v}$ that controls the evolution equations studied here.

Theorem 4.2. Suppose a solution $v=v(y, \theta, \tau)$ of equation (2.5) satisfies Assumption [A1] at $\tau=0$, and Conditions [Ca] and [CO]-[C2] for $\beta y^{2} \geq 20$ and $\tau \in\left[0, \tau_{1}\right]$. If Conditions [C0i]-[C1i] hold on the boundary of this region for $\tau \in\left[0, \tau_{1}\right]$, then for the same time interval, the solution satisfies the following estimates throughout the outer region $\left\{\beta y^{2} \geq 20\right\}$ :

$$
\begin{gathered}
v(y, \cdot, \cdot) \geq 4, \\
\left|\partial_{y} v\right| \lesssim \beta^{\frac{1}{2}} v^{\frac{1}{2}}, \\
\left|\partial_{y} v\right| \lesssim 1, \\
\left|\partial_{\theta} v\right| \leq C_{0} \kappa^{-\frac{1}{2}} v,
\end{gathered}
$$

where $C_{0}$ depends only on the initial data and not on $\tau_{1}$.

Note that estimate (4.5) provides $C$ such that $\left|\partial_{y} v\right| \leq C$ in the outer region for $\tau \in\left[0, \tau_{1}\right]$. By Condition [C0i], one has $v\left( \pm \sqrt{20} \beta^{-\frac{1}{2}}\right) \leq c^{2}$ for some $c>0$. Hence by quadrature, one obtains the immediate corollary that

$$
v=\mathcal{O}(\langle y\rangle) \text { as }|y| \rightarrow \infty .
$$

Theorem 4.2 is proved in Section 5.

In the second step, we derive estimates for second and third derivatives of $v$. It is reasonable to expect that one could bound second derivatives without assumptions 
on third derivatives, thus allowing us to "close the loop" at two derivatives in our bootstrap arguments. A reason for this expectation is that once one has suitable estimates on first derivatives, one can show that higher-order derivatives occur in combinations $\phi \cdot\left(D^{N+1} v\right)-\psi \cdot\left(D^{N+1} v\right)^{2}$, where $|\phi| \ll \psi$. Indeed, Theorem 4.2 suffices to bound the quantity $\psi>0$ from below. This expectation is correct in the outer region, where one can obtain the needed second-derivative bounds using only maximum-principle arguments. But in the inner region, more complicated machinery is needed. To construct improved second-order estimates there, we introduce and bound suitable Lyapunov functionals and then apply Sobolev embedding theorems. This method requires us to assume (and subsequently improve) pointwise bounds on third-order derivatives and $L_{\sigma}^{2}$ bounds on derivatives of orders four and five. What makes this method more effective than the maximum principle in the inner region is the fact that the Lyapunov functionals allow integration by parts.

Remark 4.3. The main reason that integration improves our estimates is the fact that for any smooth function $f$ which is orthogonal to constants in $L_{2}\left(\{y\} \times \mathbb{S}^{1}\right)$, our assumption of $\pi$-periodicity implies that

$$
\int_{\{y\} \times \mathbb{S}^{1}}\left(\partial_{\theta} f\right)^{2} \mathrm{~d} \theta \geq 4 \int_{\{y\} \times \mathbb{S}^{1}} f^{2} \mathrm{~d} \theta .
$$

Remark 4.4. Here is one reason why we need the pointwise estimates on thirdorder derivatives in [C3]. (Another reason will become clear in Section 8.) In estimating nonlinear terms in the evolution of the Lyapunov functionals introduced below, one encounters quantities of the sort $\left\langle D^{N}\left(F_{\ell}\right),\left(D^{2} v\right)\left(D^{N} v\right)\right\rangle_{\sigma}$, where the coefficients $F_{\ell}$ are given in definition (2.8). After integration by parts, one gets, schematically, $\left\langle D^{N-1}\left(F_{\ell}\right),\left(D^{3} v\right)\left(D^{N} v\right)+\left(D^{2} v\right)\left(D^{N+1} v\right)\right\rangle_{\sigma}$. Such terms are comparable to $\left\langle D^{N}(v),\left(D^{3} v\right)\left(D^{N} v\right)+\left(D^{2} v\right)\left(D^{N+1} v\right)\right\rangle_{\sigma}$. So we need $L^{\infty}$ control on third derivatives in order to impose only $L_{\sigma}^{2}$ assumptions on higher derivatives.

In Section 6.4, we prove the following result.

Theorem 4.5. Suppose that a solution $v=v(y, \theta, \tau)$ of equation (2.5) satisfies Assumption [A1] at $\tau=0$, and Conditions [Ca], [CO]-[C3], [Cs], [Cr], [Cg], and [C0i]-[C1i] for $\tau \in\left[0, \tau_{1}\right]$. Then for the same time interval, the solution satisfies the following pointwise bounds throughout the inner region $\left\{\beta y^{2} \leq 20\right\}$ :

$$
\beta\left|\partial_{y}^{2} v\right|+v^{-1}\left|\partial_{y} \partial_{\theta} v\right|+v^{-2}\left|\partial_{\theta}^{2} v\right| \lesssim \beta^{\frac{33}{20}}
$$

and

$$
\beta^{\frac{1}{2}}\left|\partial_{y}^{3} v\right|+v^{-1}\left|\partial_{y}^{2} \partial_{\theta} v\right|+v^{-2}\left|\partial_{y} \partial_{\theta}^{2} v\right|+v^{-3}\left|\partial_{\theta}^{3} v\right| \lesssim \beta^{\frac{33}{20}}
$$

The fact that we do not achieve the expected $\beta^{2}$ decay on the RHS of estimates (4.8)-(4.9) is due to our use of Sobolev embedding with respect to the weighed measure introduced in equation (2.11).

Then using Theorem 4.5 to ensure that they hold on the boundary of the inner region, we extend its estimates to the outer region.

Theorem 4.6. Suppose that a solution $v=v(y, \theta, \tau)$ of equation (2.5) satisfies Assumption [A1] at $\tau=0$, and Conditions [Ca] and [CO]-[C3] for $\beta y^{2} \geq 20$ and $\tau \in\left[0, \tau_{1}\right]$. Then the estimates

$$
\beta\left|\partial_{y}^{2} v\right|+v^{-1}\left|\partial_{y} \partial_{\theta} v\right|+v^{-2}\left|\partial_{\theta}^{2} v\right| \lesssim \beta^{\frac{33}{20}}
$$


and

$$
\beta^{\frac{1}{2}}\left|\partial_{y}^{3} v\right|+v^{-1}\left|\partial_{y}^{2} \partial_{\theta} v\right|+v^{-2}\left|\partial_{y} \partial_{\theta}^{2} v\right|+v^{-3}\left|\partial_{\theta}^{3} v\right| \lesssim \beta^{\frac{33}{20}}
$$

hold throughout the entire outer region $\left\{\beta y^{2} \geq 20\right\}$ during the same time interval, provided that they hold on the boundary $\beta y^{2}=20$.

As an easy corollary, we apply the interpolation result Lemma B.2 to get our final first-order estimate claimed in subsection 4.2 , namely

$$
\begin{aligned}
\frac{\left|\partial_{\theta} v\right|}{v^{2}} \leq \frac{1}{(1-\delta)^{2}} \frac{\left|\partial_{\theta} v_{2}\right|}{v_{1}^{2}} & \leq \frac{C_{1}}{(1-\delta)^{2}} \max _{\theta \in[0,2 \pi]} \frac{\left|\partial_{\theta}^{2} v_{2}\right|}{v_{1}^{2}} \\
& \leq C_{2} \frac{(1+\delta)^{2}}{(1-\delta)^{2}} \max _{\theta \in[0,2 \pi]} \frac{\left|\partial_{\theta}^{2} v\right|}{v^{2}} \leq C_{3} \beta^{\frac{33}{20}}
\end{aligned}
$$

Theorem 4.6 is proved in Section 7.

Finally, we improve the "smallness estimates" in Condition [C3], producing improved bounds for $\left|\partial_{y} \partial_{\theta}^{2} v\right|$ and $v^{-1}\left|\partial_{\theta}^{3} v\right|$; these serve as inputs to the second bootstrap machine constructed in Section 8 below.

Theorem 4.7. Suppose that a solution $v=v(y, \theta, \tau)$ of equation (2.5) satisfies Assumption [A1] at $\tau=0$, and Conditions [Ca], [CO]-[C3], [Cs], [Cr], [Cg], and [C0i]-[C1i] for $\tau \in\left[0, \tau_{1}\right]$. Then for the same time interval, the solution satisfies

$$
\beta^{-\frac{11}{10}}\left[\left(\partial_{y}^{3} v\right)^{2}+\left(\partial_{y}^{2} \partial_{\theta} v\right)^{2}\right]+\left(\partial_{y} \partial_{\theta}^{2} v\right)^{2}+v^{-2}\left(\partial_{\theta}^{3} v\right)^{2} \lesssim\left(\beta_{0}+\varepsilon_{0}\right)^{\frac{1}{10}}
$$

As an easy corollary, we apply Lemma B.2 to get our final second-order estimates claimed in subsection 4.2 , namely

$$
\left|\partial_{y} \partial_{\theta} v\right|+v^{-1}\left|\partial_{\theta}^{2} v\right| \lesssim\left(\beta_{0}+\varepsilon_{0}\right)^{\frac{1}{20}}
$$

Theorem 4.7 is proved in Section 7.3. Its proof completes our construction of the first bootstrap machine.

\section{Estimates OF FIRST-ORDER DERIVATIVES}

In this section, we prove the estimates that constitute Theorem 4.2. Our arguments use a version of the parabolic maximum principle adapted to noncompact domains, which we state as Proposition C.1 and prove in Appendix C.

We start with a simple observation illustrating how one applies Proposition C.1 to control inf $v$ for large $|y|$.

Lemma 5.1. If there exist constants $\varepsilon>0$ and $c \geq \sup _{0 \leq \tau \leq \tau_{1}} a^{-1 / 2}$, and a continuous function $b(\tau) \geq 0$ such that (a) $v_{0}(y, \cdot) \geq c$ for $|y| \geq \bar{b}(\overline{0})$, (b) $v( \pm b(\tau), \cdot, \tau) \geq c$ for $0 \leq \tau \leq \tau_{1}$, and (c) $v(\cdot, \cdot, \tau) \geq \varepsilon$ for $|y| \geq b(\tau)$ and $0 \leq \tau \leq \tau_{1}$, then

$$
v(y, \theta, \tau) \geq c
$$

for $|y| \geq b(\tau), 0 \leq \theta \leq 2 \pi$, and $0 \leq \tau \leq \tau_{1}$.

Proof. Apply the maximum principle in the form of Proposition C.1 to $c-v$ in the region $\Omega:=\{|y| \geq b(\tau)\}$, with $D:=\partial_{\tau}-A_{v}-B$ and $B:=a+(c v)^{-1}$. By (c), $B$ is bounded from above. Computing $D(c-v)=c^{-1}\left(1-a c^{2}\right) \leq 0$ establishes property (I). Hypotheses (a) and (b) ensure that property (II) is satisfied. Property 
(III) follows from Conditions [C0i] and [C1] by quadrature. Hence Proposition C.1 implies that $v \geq c$.

We now establish the estimates that constitute Theorem 4.2.

Proof of estimate (4.3). We apply Lemma 5.1 in the outer region $\beta y^{2} \geq 20$. Let $\varepsilon=\kappa^{-1}, c=4$ (large enough by Condition [Ca]), and $b(\tau)=\sqrt{20 \beta(\tau)^{-1}}$. Then Assumption [A2] implies property (a); Condition [C0i] gives (b); and [C0] yields (c). Thus Lemma 5.1 implies the estimate.

Proof of estimate (4.4). Set $w:=v_{1,0, \frac{1}{2}} \equiv v^{-\frac{1}{2}} \partial_{y} v$, using definition (3.1). Then by equation (3.4) in Lemma 3.1, one has

$$
\partial_{\tau} w^{2}=A_{v}\left(w^{2}\right)+\left(3 v^{-2}-a\right) w^{2}-B_{1,0, \frac{1}{2}}+2 w \sum_{\ell=0}^{4} E_{1,0, \frac{1}{2}, \ell}
$$

where the nonlinear commutator terms $E_{1,0, \frac{1}{2}, \ell}$ are defined in equation (??) and display (3.3).

We now calculate and estimate the nonlinear terms above. Observe, for example, that

$$
\begin{aligned}
E_{1,0, \frac{1}{2}, 1} & :=v^{-\frac{1}{2}} \partial_{y}\left(F_{1} \partial_{y}^{2} v\right)-F_{1} \partial_{y}^{2}\left(v^{-\frac{1}{2}} \partial_{y} v\right) \\
& =\left(\partial_{y} F_{1}\right)\left[v^{-\frac{1}{2}}\left(\partial_{y}^{2} v\right)\right]+F_{1}\left[\frac{3}{2} v^{-\frac{3}{2}}\left(\partial_{y}^{2} v\right)\left(\partial_{y} v\right)-\frac{3}{4} v^{-\frac{5}{2}}\left(\partial_{y} v\right)^{3}\right],
\end{aligned}
$$

where $^{7}$

$$
\partial_{y} F_{\ell}=\left(\partial_{p} F_{\ell}\right)\left(\partial_{y}^{2} v\right)+\left(\partial_{q} F_{\ell}\right)\left[v^{-1} \partial_{y} \partial_{\theta} v-v^{-2}\left(\partial_{y} v\right)\left(\partial_{\theta} v\right)\right] .
$$

By Lemma 3.5, there are uniform bounds $\left|\partial_{p}^{i} \partial_{q}^{j} F_{\ell}(p, q)\right| \leq C_{i, j, \ell}$. Applying this fact (which we freely use below without comment) one establishes by similar direct computations that

$$
\begin{aligned}
\left|E_{1,0, \frac{1}{2}, 0}\right| \lesssim & v^{-\frac{1}{2}}\left|\partial_{y} v\right|\left[\left|\partial_{y}^{2} v\right|+v^{-2}\left|\partial_{\theta}^{2} v\right|+v^{-1}\left|\partial_{y} \partial_{\theta} v\right|+v^{-2}\left|\partial_{\theta} v\right|\right] \\
\left|E_{1,0, \frac{1}{2}, 1}\right| \lesssim & {\left[v^{-\frac{1}{2}}\left|\partial_{y}^{2} v\right|+v^{-\frac{3}{2}}\left|\partial_{y}^{2} v\right|\left|\partial_{y} v\right|+v^{-\frac{5}{2}}\left|\partial_{y} v\right|^{3}\right] } \\
\left|E_{1,0, \frac{1}{2}, 2}\right| \lesssim & {\left[v^{-\frac{5}{2}}\left|\partial_{\theta}^{2} v\right|+v^{-\frac{7}{2}}\left(\left|\partial_{y} v\right|\left|\partial_{\theta}^{2} v\right|+\left|\partial_{y} \partial_{\theta} v\right|\left|\partial_{\theta} v\right|\right)+v^{-\frac{9}{2}}\left|\partial_{y} v \| \partial_{\theta} v\right|^{2}\right] } \\
\left|E_{1,0, \frac{1}{2}, 3}\right| \lesssim & {\left[v^{-\frac{3}{2}}\left|\partial_{y}^{2} v \| \partial_{y} \partial_{\theta} v\right|+v^{-\frac{5}{2}}\left(\left|\partial_{y} \partial_{\theta} v\right|^{2}+\left|\partial_{y} \partial_{\theta} v \| \partial_{y} v\right|+\left|\partial_{y}^{2} v\right|^{\mid} \partial_{\theta} v \mid\right)\right.} \\
& \left.+v^{-\frac{7}{2}}\left(\left|\partial_{y} \partial_{\theta} v \| \partial_{y} v\right|+\left|\partial_{y} v\right|^{2}\left|\partial_{\theta} v\right|\right)\right] \\
\left|E_{1,0, \frac{1}{2}, 4}\right| \lesssim & {\left[v^{-\frac{5}{2}}\left|\partial_{y}^{2} v\left\|\partial_{\theta} v\left|+v^{-\frac{7}{2}}\left(\left|\partial_{y} v\left\|\partial_{\theta} v|+| \partial_{y} \partial_{\theta} v\right\| \partial_{\theta} v\right|\right)+v^{-\frac{9}{2}}\right| \partial_{y} v\right\| \partial_{\theta} v\right|^{2}\right] }
\end{aligned}
$$

By Conditions [C1]-[C2], one has $\left|\partial_{y} v\right| \lesssim v^{\frac{1}{2}} \beta^{\frac{2}{5}},\left|\partial_{\theta} v\right| \lesssim v^{2} \beta^{\frac{3}{2}},\left|\partial_{y}^{2} v\right| \lesssim \beta^{\frac{3}{5}}$, $\left|\partial_{y} \partial_{\theta} v\right| \lesssim v \beta^{\frac{3}{2}},\left|\partial_{y} \partial_{\theta} v\right| \lesssim 1$, and $\left|\partial_{\theta}^{2} v\right| \lesssim v^{2} \beta^{\frac{3}{2}}$. Combining these inequalities and using estimate (4.3), one readily obtains

$$
2\left|w \sum_{\ell=0}^{4} E_{1,0, \frac{1}{2}, \ell}\right| \leq C \beta^{\frac{3}{5}}|w| .
$$

\footnotetext{
${ }^{7}$ Recall from definition (2.7) that $p=\partial_{y} v$ and $q=v^{-1} \partial_{\theta} v$.
} 
By Condition [Ca] and estimate (4.3), one has $\left(3 v^{-2}-a\right) w^{2} \leq-\frac{1}{4} w^{2}$ in the outer region, for $\kappa$ large enough, whereupon completing the square shows that

$$
C \beta^{\frac{3}{5}}|w|-\frac{1}{8} w^{2} \leq 2 C^{2} \beta^{\frac{6}{5}} .
$$

Now let $\Gamma>0$ be a large constant to be chosen below. Using Corollary 3.3 to see that $B_{1,0, \frac{1}{2}} \geq 0$, and applying the estimates above, one obtains

$$
\partial_{\tau}\left(w^{2}-\Gamma \beta\right) \leq A_{v}\left(w^{2}-\Gamma \beta\right)-\frac{1}{8}\left(w^{2}-\Gamma \beta\right)+\left(\beta+\frac{2 C^{2}}{\Gamma} \beta^{\frac{1}{5}}-\frac{1}{8}\right) \Gamma \beta .
$$

We shall apply Proposition C.1. By taking $\kappa$ and $\Gamma$ large enough, we can ensure that

$$
\partial_{\tau}\left(w^{2}-\Gamma \beta\right) \leq A_{v}\left(w^{2}-\Gamma \beta\right)-\frac{1}{8}\left(w^{2}-\Gamma \beta\right)
$$

which is property (I). Making $\Gamma$ larger if necessary, we can by our Main Assumptions ensure that $w^{2} \leq \Gamma \beta$ at $\tau=0$, whereupon property (II) follows from Condition [C1i] on the boundary. Property (III) is a consequence of Condition [COi] on the boundary and Condition [C1] in the outer regon. Hence we have $w^{2} \leq \Gamma \beta$ in the outer region for $0 \leq \tau \leq \tau_{1}$ by the maximum principle.

Proof of estimate (4.5). Arguing as in the proof of Corollary 3.3, one computes from equation (3.4), equation (??), and definition (3.3), that

$$
\partial_{\tau}\left(\partial_{y} v\right)^{4} \leq A_{v}\left(\partial_{y} v\right)^{4}+4 v^{-2}\left(\partial_{y} v\right)^{4}+4\left(\partial_{y} v\right)^{3} \sum_{\ell=1}^{4} E_{1,0,0, \ell}
$$

By estimate (4.4) and the implications of Conditions [C1]-[C2] that $\left|\partial_{\theta} v\right| \lesssim v^{2} \beta^{\frac{3}{2}}$, $\left|\partial_{\theta} v\right| \lesssim v,\left|\partial_{y}^{2} v\right| \lesssim \beta^{\frac{3}{5}},\left|\partial_{y} \partial_{\theta} v\right| \lesssim v \beta^{\frac{3}{2}},\left|\partial_{y} \partial_{\theta} v\right| \lesssim 1$, and $\left|\partial_{\theta}^{2} v\right| \lesssim v^{2} \beta^{\frac{3}{2}}$, one can estimate that $\left|E_{1,0,0,1}\right| \lesssim \beta^{\frac{6}{5}}$, with $\left|E_{1,0,0, \ell}\right| \lesssim \beta^{2}$ for $\ell=2,3,4$.

Define $w:=\left(\partial_{y} v\right)^{4}+1$. Then by estimate (4.4) and Young's inequality, one has

$$
\begin{aligned}
\partial_{\tau} w & \leq A_{v}(w)+C_{1} \beta^{2}+C_{2} \beta^{\frac{6}{5}}\left[\left(\partial_{y} v\right)^{4}+1\right] \\
& \leq A_{v}(w)+C \beta^{\frac{6}{5}} w .
\end{aligned}
$$

Let $\varphi(\tau)$ solve the ODE $\varphi^{\prime}=C \beta^{\frac{6}{5}} \varphi$ with initial condition $\varphi(0)=C_{0}$, where $C_{0}$ may be chosen by our Main Assumptions so that $w \leq C_{0}$ at $\tau=0$. Then

$$
\partial_{\tau}(w-\varphi) \leq A_{v}(w-\varphi)+C \beta^{\frac{6}{5}}(w-\varphi) .
$$

Properties (I)-(II) of Proposition C.1 are clearly satisfied for this equation, while property (III) follows from estimate (4.4). Hence we have $w \leq \varphi$ by the maximum principle. Because $\varphi$ is uniformly bounded in time, the lemma follows.

Proof of estimate (4.6). Let $w:=v_{0,1,1} \equiv v^{-1} \partial_{\theta} v$. By Corollary 3.3, $w^{2}$ satisfies the differential inequality

$$
\partial_{\tau} w^{2} \leq A_{v}\left(w^{2}\right)+4 v^{-2} w^{2}+2\left|w \sum_{\ell=0}^{4} E_{0,1,1, \ell}\right| .
$$

We proceed to bound the reaction terms. By Condition [C1], one has

$$
v^{-2} w^{2} \lesssim \beta^{3}
$$


By Conditions [C1]-[C2], one has $\left|E_{0,1,1,0}\right| \lesssim \beta^{\frac{21}{10}}$. By direct computation (compare estimate (6.4) below), one has $v^{-1}\left|\partial_{\theta} F_{\ell}\right| \lesssim \beta^{\frac{3}{2}}$. Thus one gets $\left|E_{0,1,1,1}\right| \lesssim \beta^{2}$ and $\left|E_{0,1,1, \ell}\right| \lesssim \beta^{3}$ for $\ell=2,3,4$. By Condition [C1], one has $|w| \lesssim 1$, and thus $\partial_{\tau} w^{2} \leq A_{v}\left(w^{2}\right)+C \beta^{2}$ for some $C<\infty$.

Now let $\varphi(\tau)$ solve $\varphi^{\prime}=C \beta^{2}$ with $\varphi(0)=C_{*} \kappa^{-1}$. By our Main Assumptions, $w \leq \varphi$ at $\tau=0$ for $C_{*}>0$ sufficiently large and depending only on the initial data. Because

$$
\partial_{\tau}\left(w^{2}-\varphi\right) \leq A_{v}\left(w^{2}-\varphi\right)
$$

the maximum principle implies that $w^{2} \leq \varphi$ for as long as the solution exists.

The proof of Theorem 4.2 is now complete.

\section{Decay estimates in the INNER REgion}

In this section, we construct the machinery that will prove Theorem 4.5.

Given integers $m, n \geq 0$, we define functionals $\Omega_{m, n}=\Omega_{m, n}(\tau)$ by

$$
\Omega_{m, n}:=\int_{-\infty}^{\infty} \int_{0}^{2 \pi} v_{m, n, n}^{2} \mathrm{~d} \theta \sigma \mathrm{d} y \equiv \int_{-\infty}^{\infty} \int_{0}^{2 \pi} v^{-2 n}\left|\partial_{y}^{m} \partial_{\theta}^{n} v\right|^{2} \mathrm{~d} \theta \sigma \mathrm{d} y,
$$

where the notation $v_{m, n, k}$ appears in equation (3.1), and $\sigma=\sigma(y)$ is defined in equation (2.11). By Conditions [C2], [C3], and [Cs], these functionals and their $\tau$-derivatives are well defined if $m+n \leq 5$.

Our strategy to prove Theorem 4.5 consists of three steps. (I) We bound weighted sums of $\Omega_{m, n}$ with $2 \leq m+n \leq 3$. (II) We bound weighted sums of $\Omega_{m, n}$ with $4 \leq m+n \leq 5$. (III) We apply Sobolev embedding, using the facts that $|y| \lesssim \beta^{-\frac{1}{2}}$ in the inner region, and that $\sigma \sim|y|^{-\frac{6}{5}}$ as $|y| \rightarrow \infty$.

A consequence of this strategy is that we only need strong estimates for secondorder derivatives. It suffices to show that higher derivatives decay at the same rates as those of second order, rather than at the faster rates one would expect from parabolic smoothing. This somewhat reduces the work necessary to bound derivatives of orders three through five.

6.1. Differential inequalities. We first derive differential inequalities satisfied by the squares of the second-order quantities appearing in Theorem 4.5. To avoid later redundancy, these estimates are designed to be useful in both the inner and outer regions. They could be obtained using the more general techniques developed in Appendix D and employed below to bound higher derivatives. We chose to derive them explicitly here, both to obtain the sharpest results possible and to help the reader by introducing our methods in as transparent a manner as we can.

Observe that Conditions [C0i] and [C1i] imply that inequalities (4.4)-(4.6) of Theorem 4.2 hold in the inner region as well as the outer region. Thus we assume in this subsection that those inequalities hold globally.

Lemma 6.1. There exist $0<\varepsilon<C<\infty$ such that the quantity $\left|\partial_{y}^{2} v\right|^{2}$ satisfies

$$
\begin{aligned}
\partial_{\tau}\left|\partial_{y}^{2} v\right|^{2} & \leq A_{v}\left(\left|\partial_{y}^{2} v\right|^{2}\right)+X\left|\partial_{y}^{2} v\right|^{2}-Y\left(\left|\partial_{y}^{3} v\right|^{2}+v^{-2}\left|\partial_{y}^{2} \partial_{\theta} v\right|^{2}\right) \\
& +C\left(\beta^{\frac{11}{5}}+\beta\left|\partial_{y}^{2} v\right|+\beta^{\frac{11}{10}} v^{-2}\left|\partial_{y} \partial_{\theta}^{2} v\right|\right),
\end{aligned}
$$

where in the inner region $\left\{\beta y^{2} \leq 20\right\}$,

$$
X=2\left(v^{-2}-a\right) \quad \text { and } \quad Y \geq \frac{2}{1+\varepsilon},
$$


while in the outer region,

$$
X \leq \frac{1}{8}-2 a \leq-\frac{3}{4} \quad \text { and } \quad Y \geq \varepsilon .
$$

Proof. Define $w:=v_{2,0,0} \equiv \partial_{y}^{2} v$. Then by equation (3.4) and Corollary 3.3, one has

$$
\partial_{\tau} w^{2} \leq A_{v}\left(w^{2}\right)+X w^{2}-Y\left(\left|\partial_{y}^{3} v\right|^{2}+v^{-2}\left|\partial_{y}^{2} \partial_{\theta} v\right|^{2}\right)+2\left|w \sum_{\ell=1}^{5} E_{2,0,0, \ell}\right|,
$$

where $X:=2\left(v^{-2}-a\right)$ and $Y:=\frac{2}{1+p^{2}+q^{2}}$.

The estimates for $X$ in the outer region follow from estimate (4.3) for $v$ there, and Condition $[\mathrm{Ca}]$.

Although we discarded analogous quantities $B_{m, n, k}$ in the proof of Theorem 4.2, we retain them above to help us control third-order derivatives of $v$. By Corollary 3.3, estimate (4.5) for $p=\partial_{y} v$, and estimate (4.6) for $q=v^{-1} \partial_{\theta} v$, there exists $\varepsilon>0$ such that $B_{2,0,0} \geq 2 \varepsilon\left(\left|\partial_{y}^{3} v\right|^{2}+v^{-2}\left|\partial_{y}^{2} \partial_{\theta} v\right|^{2}\right)$ in the outer region. (Compare Remark 3.4.)

In the inner region, one can do better. Condition [C0i] gives uniform bounds for $v$ there, whence Condition [C1i] implies that $|p|=\left|\partial_{y} v\right| \lesssim \beta^{\frac{1}{2}}$ and similarly that $|q|=\left|v^{-1} \partial_{\theta} v\right| \lesssim \beta^{\frac{3}{2}}$ in the inner region. Hence

$$
B_{2,0,0} \geq\left(\frac{2}{1+\varepsilon}+\varepsilon\right)\left(\left|\partial_{y}^{3} v\right|^{2}+v^{-2}\left|\partial_{y}^{2} \partial_{\theta} v\right|^{2}\right)
$$

in the inner region.

We next estimate the $E_{2,0,0, \ell}$ terms above. By Conditions $[\mathrm{C} 1]-[\mathrm{C} 2]$ and estimate (4.5), one has

$$
\left|\partial_{y} F_{\ell}\right|=\left|\left(\partial_{p} F_{\ell}\right)\left(\partial_{y}^{2} v\right)+\left(\partial_{q} F_{\ell}\right)\left(v^{-1} \partial_{y} \partial_{\theta} v-v^{-2} \partial_{y} v \partial_{\theta} v\right)\right| \lesssim \beta^{\frac{3}{5}} .
$$

Next one calculates that

$$
\begin{aligned}
\partial_{y}^{2} F_{\ell}= & \left(\partial_{p}^{2} F_{\ell}\right)\left(\partial_{y}^{2} v\right)^{2}+2\left(\partial_{p} \partial_{q} F_{\ell}\right)\left(\partial_{y}^{2} v\right)\left(v^{-1} \partial_{y} \partial_{\theta} v-v^{-2} \partial_{y} v \partial_{\theta} v\right) \\
& +\left(\partial_{q}^{2} F_{\ell}\right)\left(v^{-1} \partial_{y} \partial_{\theta} v-v^{-2} \partial_{y} v \partial_{\theta} v\right)^{2}+\left(\partial_{p} F_{\ell}\right)\left(\partial_{y}^{3} v\right) \\
& +\left(\partial_{q} F_{\ell}\right)\left[v^{-1} \partial_{y}^{2} \partial_{\theta} v-2 v^{-2} \partial_{y} \partial_{\theta} v \partial_{y} v-v^{-2} \partial_{y}^{2} v \partial_{\theta} v+2 v^{-3}\left(\partial_{y} v\right)^{2} \partial_{\theta} v\right]
\end{aligned}
$$

and uses Conditions [C1]-[C2] with inequalities (4.4)-(4.5) from Theorem 4.2 to estimate

$$
\left|\partial_{y}^{2} F_{\ell}\right| \lesssim \beta^{\frac{6}{5}}+\left|\partial_{y}^{3} v\right|+v^{-1}\left|\partial_{y}^{2} \partial_{\theta} v\right|
$$

Collecting the estimates above and using Condition [C2] again, one obtains

$$
\left|E_{2,0,0,1}\right|=\left|\left(\partial_{y}^{2} F_{1}\right)\left(\partial_{y}^{2} v\right)+2\left(\partial_{y} F_{1}\right)\left(\partial_{y}^{3} v\right)\right| \lesssim \beta^{\frac{9}{5}}+\beta^{\frac{3}{5}}\left(\left|d y^{3} v\right|+v^{-1}\left|\partial_{y}^{2} \partial_{\theta} v\right|\right) .
$$

In similar fashion, one derives

$$
\left|E_{2,0,0,2}\right| \lesssim \beta^{2}+\beta^{\frac{3}{2}}\left(\left|\partial_{y}^{3} v\right|+v^{-1}\left|\partial_{y}^{2} \partial_{\theta} v\right|\right)+\beta^{\frac{1}{2}} v^{-2}\left|\partial_{y} \partial_{\theta}^{2} v\right|
$$

and

Noting that

$$
\left|E_{2,0,0,3}\right| \lesssim \beta^{\frac{21}{10}}+\beta^{\frac{3}{2}}\left|\partial_{y}^{3} v\right|+\beta^{\frac{1}{2}} v^{-1}\left|\partial_{y}^{2} \partial_{\theta} v\right| \cdot
$$

$$
\begin{aligned}
E_{2,0,0,4}= & \left(\partial_{y}^{2} F_{4}\right)\left(v^{-2} \partial_{\theta} v\right)+\left(\partial_{y} F_{4}\right)\left(2 v^{-2} \partial_{y} \partial_{\theta} v-4 v^{-3} \partial_{y} v \partial_{\theta} v\right) \\
& -F_{4}\left[4 v^{-3} \partial_{y} \partial_{\theta} v \partial_{y} v+2 v^{-3} \partial_{y}^{2} v \partial_{\theta} v-6 v^{-4}\left(\partial_{y} v\right)^{2} \partial_{\theta} v\right],
\end{aligned}
$$


one applies Condition [C2] and estimate (4.4) to get

$$
\left|E_{2,0,0,4}\right| \lesssim \beta^{2}+\beta^{\frac{3}{2}}\left(\left|\partial_{y}^{3} v\right|+v^{-1}\left|\partial_{y}^{2} \partial_{\theta} v\right|\right) .
$$

By estimate (4.4) again, one has

$$
\left|E_{2,0,0,5}\right|=2 v^{-3}\left(\partial_{y} v\right)^{2} \lesssim \beta
$$

Collecting the estimates above and using Condition [C2], we conclude that

$$
\left|w \sum_{\ell=1}^{5} E_{2,0,0, \ell}\right| \lesssim \beta|w|+\beta^{\frac{11}{10}}\left(\left|\partial_{y}^{3} v\right|+v^{-1}\left|\partial_{y}^{2} \partial_{\theta} v\right|+v^{-2}\left|\partial_{y} \partial_{\theta}^{2} v\right|\right) .
$$

Using Remark 3.4 and completing squares to see that $\beta^{\frac{11}{10}}\left|\partial_{y}^{3} v\right|-\varepsilon\left|\partial_{y}^{3} v\right|^{2} \leq \frac{1}{4 \varepsilon} \beta^{\frac{11}{5}}$ and $\beta^{\frac{11}{10}} v^{-1}\left|\partial_{y}^{2} \partial_{\theta} v\right|-\varepsilon v^{-2}\left|\partial_{y}^{2} \partial_{\theta} v\right|^{2} \leq \frac{1}{4 \varepsilon} \beta^{\frac{11}{5}}$, we combine the estimates above to obtain the lemma.

Lemma 6.2. There exist $0<\varepsilon<C<\infty$ such that the quantity $v^{-2}\left|\partial_{y} \partial_{\theta} v\right|^{2}$ satisfies

$$
\begin{aligned}
\partial_{\tau}\left(v^{-2}\left|\partial_{y} \partial_{\theta} v\right|^{2}\right) & \leq A_{v}\left(v^{-2}\left|\partial_{y} \partial_{\theta} v\right|^{2}\right)+X v^{-2}\left|\partial_{y} \partial_{\theta} v\right|^{2}-Y\left(v^{-2}\left|\partial_{y}^{2} \partial_{\theta} v\right|^{2}+v^{-4}\left|\partial_{y} \partial_{\theta}^{2} v\right|^{2}\right) \\
& +C\left(\beta^{4}+\beta^{2} v^{-1}\left|\partial_{y} \partial_{\theta} v\right|+\beta^{3}\left|\partial_{y}^{3} v\right|+\beta^{2} v^{-3}\left|\partial_{\theta}^{3} v\right|\right)
\end{aligned}
$$

where in the inner region $\left\{\beta y^{2} \leq 20\right\}$,

$$
X=2\left(2 v^{-2}-a\right)+\varepsilon \quad \text { and } \quad Y \geq \frac{1}{1+\varepsilon},
$$

while in the outer region,

$$
X \leq \frac{1}{4}-2 a+\varepsilon \leq-\frac{5}{8} \quad \text { and } \quad Y \geq \varepsilon .
$$

Proof. Define $w:=v_{1,1,1} \equiv v^{-1} \partial_{y} \partial_{\theta} v$. Then by equation (3.4) and estimate (3.5), one has

$$
\partial_{\tau} w^{2} \leq A_{v}\left(w^{2}\right)+X w^{2}-Y\left(v^{-2}\left|\partial_{y}^{2} \partial_{\theta} v\right|^{2}+v^{-4}\left|\partial_{y} \partial_{\theta}^{2} v\right|^{2}\right)+2\left|w \sum_{\ell=0}^{5} E_{1,1,1, \ell}\right|,
$$

where $X:=2\left(2 v^{-2}-a\right)+\varepsilon$ and $Y:=\frac{1}{1+p^{2}+q^{2}}$. As explained in Remark 3.4, existence of $0<\varepsilon \ll 1$ follows easily from (3.5) and the estimates for $\left|v_{1,0,1}\right|$ and $\left|v_{0,1,1}\right|$ implied by Theorem 4.2. The estimates for $X$ in the outer region follow from estimate (4.3) for $v$ there, and Condition [Ca]. The estimates for $Y$ are the same as those in Lemma 6.1 above.

Using Condition [C2], it is easy to see that $\left|E_{1,1,1,0}\right| \lesssim \beta^{\frac{21}{10}}$. We compute and use Conditions [C1]-[C2] to estimate

$$
v^{-1}\left|\partial_{\theta} F_{\ell}\right|=v^{-1}\left|\left(\partial_{p} F_{\ell}\right)\left(\partial_{y} \partial_{\theta} v\right)+\left(\partial_{q} F_{\ell}\right)\left[v^{-1} \partial_{\theta}^{2} v-v^{-2}\left(\partial_{\theta} v\right)^{2}\right]\right| \lesssim \beta^{\frac{3}{2}} .
$$

As we did in the proof of Lemma 6.1, we next compute and estimate

$$
v^{-1}\left|\partial_{y} \partial_{\theta} F_{\ell}\right| \lesssim \beta^{2}+v^{-1}\left|\partial_{y}^{2} \partial_{\theta} v\right|+v^{-2}\left|\partial_{y} \partial_{\theta}^{2} v\right|
$$

Here we used inequality (4.4) as well. 
Using the inequalities above and estimate (6.2), one sees, for example, that

$$
\begin{aligned}
E_{1,1,1,3}= & \left(v^{-1} \partial_{y} \partial_{\theta} F_{3}\right)\left(v^{-1} \partial_{y} \partial_{\theta} v\right) \\
& +\left(v^{-1} \partial_{\theta} F_{3}\right)\left(v^{-1} \partial_{y}^{2} \partial_{\theta} v-v^{-2} \partial_{y} \partial_{\theta} v \partial_{y} v\right) \\
& +\left(\partial_{y} F_{3}\right)\left(v^{-2} \partial_{y} \partial_{\theta}^{2} v-v^{-3} \partial_{y} \partial_{\theta} v \partial_{\theta} v\right)
\end{aligned}
$$

may be estimated by

$$
\left|E_{1,1,1,3}\right| \lesssim \beta^{\frac{7}{2}}+\beta^{\frac{3}{2}} v^{-1}\left|\partial_{y}^{2} \partial_{\theta} v\right|+\beta^{\frac{3}{5}} v^{-2}\left|\partial_{y} \partial_{\theta}^{2} v\right| .
$$

In like fashion, we derive the estimates

$$
\begin{aligned}
& \left|E_{1,1,1,1}\right| \lesssim \beta^{\frac{21}{10}}+\beta^{\frac{3}{2}}\left|\partial_{y}^{3} v\right|+\beta^{\frac{1}{2}}\left(v^{-1}\left|\partial_{y}^{2} \partial_{\theta} v\right|+v^{-2}\left|\partial_{y} \partial_{\theta}^{2} v\right|\right), \\
& \left|E_{1,1,1,2}\right| \lesssim \beta^{3}+\beta^{\frac{3}{2}}\left(v^{-1}\left|\partial_{y}^{2} \partial_{\theta} v\right|+v^{-2}\left|\partial_{y} \partial_{\theta}^{2} v\right|\right)+\beta^{\frac{1}{2}} v^{-3}\left|\partial_{\theta}^{3} v\right|, \\
& \left|E_{1,1,1,4}\right| \lesssim \beta^{2}+\beta^{\frac{3}{2}}\left(v^{-1}\left|\partial_{y}^{2} \partial_{\theta} v\right|+v^{-2}\left|\partial_{y} \partial_{\theta}^{2} v\right|\right) .
\end{aligned}
$$

We omit some details which are entirely analogous to those shown above. Finally, Condition [C1] and estimate (4.4) give

$$
\left|E_{1,1,1,5}\right|=2 v^{-3}\left|\partial_{y} v\right|\left|\partial_{\theta} v\right| \lesssim \beta^{2} .
$$

Collecting the estimates above and using Condition [C2], we conclude that

$$
\left|w \sum_{\ell=0}^{5} E_{1,1,1, \ell}\right| \lesssim \beta^{2}|w|+\beta^{3}\left|\partial_{y}^{3} v\right|+\beta^{2}\left(v^{-1}\left|\partial_{y}^{2} \partial_{\theta} v\right|+v^{-2}\left|\partial_{y} \partial_{\theta}^{2} v\right|+v^{-3}\left|\partial_{\theta}^{3} v\right|\right) .
$$

Using Remark 3.4 and completing squares to get $\beta^{2} v^{-1}\left|\partial_{y}^{2} \partial_{\theta} v\right|-\varepsilon v^{-2}\left|\partial_{y}^{2} \partial_{\theta} v\right|^{2} \leq$ $\frac{1}{4 \varepsilon} \beta^{4}$ and $\beta^{2} v^{-2}\left|\partial_{y} \partial_{\theta}^{2} v\right|-\varepsilon v^{-4}\left|\partial_{y} \partial_{\theta}^{2} v\right|^{2} \leq \frac{1}{4 \varepsilon} \beta^{4}$, we combine the estimates above to obtain the lemma.

Lemma 6.3. There exist $0<\varepsilon<C<\infty$ such that the quantity $v^{-4}\left|\partial_{\theta}^{2} v\right|^{2}$ satisfies

$$
\begin{aligned}
\partial_{\tau}\left(v^{-4}\left|\partial_{\theta}^{2} v\right|^{2}\right) & \leq A_{v}\left(v^{-4}\left|\partial_{\theta}^{2} v\right|^{2}\right)+X v^{-4}\left|\partial_{\theta}^{2} v\right|^{2}-Y\left(v^{-4}\left|\partial_{y} \partial_{\theta}^{2} v\right|^{2}+v^{-6}\left|\partial_{\theta}^{3} v\right|^{2}\right) \\
& +C\left(\beta^{4}+\beta^{\frac{21}{10}} v^{-2}\left|\partial_{\theta}^{2} v\right|+\beta^{3} v^{-1}\left|\partial_{y}^{2} \partial_{\theta} v\right|\right)
\end{aligned}
$$

where in the inner region $\left\{\beta y^{2} \leq 20\right\}$,

$$
X=2\left(3 v^{-2}-a\right)+\varepsilon \quad \text { and } \quad Y \geq \frac{1}{1+\varepsilon},
$$

while in the outer region,

$$
X \leq \frac{3}{8}-2 a+\varepsilon \leq-\frac{1}{2} \quad \text { and } \quad Y \geq \varepsilon .
$$

Proof. Define $w:=v_{0,2,2} \equiv v^{-2} \partial_{\theta}^{2} v$. Then by equation (3.4) and estimate (3.5), one has

$$
\partial_{\tau} w^{2} \leq A_{v}\left(w^{2}\right)+X w^{2}-Y\left(v^{-4}\left|\partial_{y} \partial_{\theta}^{2} v\right|^{2}+v^{-6}\left|\partial_{\theta}^{3} v\right|^{2}\right)+2\left|w \sum_{\ell=0}^{5} E_{0,2,2, \ell}\right|,
$$

where $X:=2\left(3 v^{-2}-a\right)+\varepsilon$ and $Y:=\frac{1}{1+p^{2}+q^{2}}$. The estimates for $X$ in the outer region follow from estimate (4.3) for $v$ there, and Condition [Ca]. The estimates for $Y$ are the same as those in Lemma 6.1. 
To proceed, we compute

$$
\begin{aligned}
\partial_{\theta}^{2} F_{\ell}= & \left(\partial_{p}^{2} F_{\ell}\right)\left(\partial_{y} \partial_{\theta} v\right)^{2} \\
& +2\left(\partial_{p} \partial_{q} F_{\ell}\right)\left(\partial_{y} \partial_{\theta} v\right)\left[v^{-1} \partial_{\theta}^{2} v-v^{-2}\left(\partial_{\theta} v\right)^{2}\right] \\
& +\left(\partial_{q}^{2} F_{\ell}\right)\left[v^{-1} \partial_{\theta}^{2} v-v^{-2}\left(\partial_{\theta} v\right)^{2}\right]^{2} \\
& +\left(\partial_{p} F_{\ell}\right)\left(\partial_{y} \partial_{\theta}^{2} v\right)+\left(\partial_{q} F_{\ell}\right) \partial_{\theta}\left[v^{-1} \partial_{\theta}^{2} v-v^{-2}\left(\partial_{\theta} v\right)^{2}\right],
\end{aligned}
$$

which we estimate in the form

$$
v^{-2}\left|\partial_{\theta}^{2} F_{\ell}\right| \lesssim \beta^{3}+v^{-2}\left|\partial_{y} \partial_{\theta}^{2} v\right|+v^{-3}\left|\partial_{\theta}^{3} v\right| .
$$

By Conditions [C1]-[C2], it is easy to see that $\left|E_{0,2,2,0}\right| \lesssim \beta^{\frac{21}{10}}$. Using [C1]-[C2], estimate (6.4) for $v^{-1}\left|\partial_{\theta} F_{1}\right|$, and estimate (6.6) for $v^{-2}\left|\partial_{\theta}^{2} F_{1}\right|$, one can estimate

$$
\begin{aligned}
E_{0,2,2,1}= & \left(v^{-2} \partial_{\theta}^{2} F_{1}\right)\left(\partial_{y}^{2} v\right)+2\left(v^{-1} \partial_{\theta} F_{1}\right)\left(v^{-1} \partial_{y}^{2} \partial_{\theta} v\right) \\
& -F_{1}\left[2 \partial_{y}\left(v^{-2}\right)\left(\partial_{y} \partial_{\theta}^{2} v\right)+\partial_{y}^{2}\left(v^{-2}\right) \partial_{\theta}^{2} v\right]
\end{aligned}
$$

by

$$
\left|E_{0,2,2,1}\right| \lesssim \beta^{\frac{21}{10}}+\beta^{\frac{3}{2}} v^{-1}\left|\partial_{y}^{2} \partial_{\theta} v\right|+\beta^{\frac{1}{2}} v^{-2}\left|\partial_{y} \partial_{\theta}^{2} v\right|+\beta^{\frac{3}{5}} v^{-3}\left|\partial_{\theta}^{3} v\right| .
$$

Continuing in this fashion, one proceeds to estimate the remaining terms,

$$
\begin{aligned}
& \left|E_{0,2,2,2}\right| \lesssim \beta^{\frac{9}{2}}+\beta^{\frac{3}{2}}\left(v^{-2}\left|\partial_{y} \partial_{\theta}^{2} v\right|+v^{-3}\left|\partial_{\theta}^{3} v\right|\right), \\
& \left|E_{0,2,2,3}\right| \lesssim \beta^{3}+\beta^{\frac{3}{2}} v^{-2}\left|\partial_{y} \partial_{\theta}^{2} v\right|+\beta^{\frac{1}{2}} v^{-3}\left|\partial_{\theta}^{3} v\right|, \\
& \left|E_{0,2,2,4}\right| \lesssim \beta^{3}+\beta^{\frac{3}{2}}\left(v^{-2}\left|\partial_{y} \partial_{\theta}^{2} v\right|+v^{-3}\left|\partial_{\theta}^{3} v\right|\right), \\
& \left|E_{0,2,2,5}\right| \lesssim \beta^{3} .
\end{aligned}
$$

Collecting the estimates above and using Condition [C2], we conclude that

$$
\left|w \sum_{\ell=0}^{5} E_{0,2,2, \ell}\right| \lesssim \beta^{\frac{21}{10}}|w|+\beta^{3} v^{-1}\left|\partial_{y}^{2} \partial_{\theta} v\right|+\beta^{2}\left(v^{-2}\left|\partial_{y} \partial_{\theta}^{2} v\right|+v^{-3}\left|\partial_{\theta}^{3} v\right|\right) .
$$

The lemma again follows by recalling Remark 3.4 and completing the squares.

6.2. Lyapunov functionals of second and third order. In this subsection, we derive differential inequalities satisfied by the $\Omega_{m, n}$ with $2 \leq m+n \leq 3$. In the next subsection, we treat the cases $4 \leq m+n \leq 5$. Our arguments are slightly different for $m+n=2$ than they are for $3 \leq m+n \leq 5$. But in all cases, we shall exploit the fact that as $\Sigma \rightarrow \infty$, one has

$$
\left\|\sigma^{-1} \partial_{y}^{2} \sigma\right\|_{\infty}=\mathcal{O}\left(\Sigma^{-1}\right) \text { and }\left\|\sigma^{-1} \partial_{y} \sigma\right\|_{\infty}=\mathcal{O}\left(\Sigma^{-\frac{1}{2}}\right),
$$

where $\sigma(y):=\left(\Sigma+y^{2}\right)^{-\frac{3}{5}}$ is defined in equation (2.11).

Remark 6.4. To derive good differential inequalities for the $\Omega_{m, n}$, we shall need to use (6.7) by taking $\Sigma$ sufficiently large. See in particular the estimates derived in display (6.8) below. On the other hand, the fact that one can make $\rho$ arbitrarily small in Lemmata 6.5-6.9 and 6.11-6.12 by making $\Sigma$ large is an interesting feature of the proof but is not needed for the arguments elsewhere in this paper. 
To estimate the evolution of the second-order functionals $\Omega_{m, n}$ with $m+n=2$, we can recycle the estimates derived in Lemmata 6.1-6.3.

Lemma 6.5. There exist constants $\varepsilon>0$ and $\rho=\rho(\Sigma)>0$, with $\rho(\Sigma) \searrow 0$ as $\Sigma \rightarrow \infty$, such that

$$
\frac{d}{d \tau} \Omega_{1,1} \leq-\varepsilon\left(\Omega_{1,1}+\Omega_{2,1}+\Omega_{1,2}\right)+\rho \beta^{2}\left(\Omega_{1,1}^{\frac{1}{2}}+\beta \Omega_{3,0}^{\frac{1}{2}}+\Omega_{2,1}^{\frac{1}{2}}+\Omega_{1,2}^{\frac{1}{2}}+\Omega_{0,3}^{\frac{1}{2}}\right) .
$$

Proof. Let $w:=v_{1,1,1} \equiv v^{-1} \partial_{y} \partial_{\theta} v$. Condition [Cs] lets us apply dominated convergence to compute $\frac{d}{d \tau} \Omega_{1,1}$, whence Lemma 3.1 gives

$$
\begin{aligned}
\frac{1}{2} \frac{d}{d \tau} \Omega_{1,1}= & \int_{\mathbb{R}} \int_{\mathbb{S}^{1}} w A_{v}(w) \mathrm{d} \theta \sigma \mathrm{d} y \\
& +\int_{\mathbb{R}} \int_{\mathbb{S}^{1}}\left(2 v^{-2}-a\right) w^{2} \mathrm{~d} \theta \sigma \mathrm{d} y \\
& +\int_{\mathbb{R}} \int_{\mathbb{S}^{1}}\left(\sum_{\ell=0}^{5} E_{1,1,1, \ell}\right) w \mathrm{~d} \theta \sigma \mathrm{d} y \\
= & : I_{1}+I_{2}+I_{3} .
\end{aligned}
$$

By Lemma 6.2 and its proof, one has

$$
I_{2} \leq \int_{\beta y^{2} \leq 20} \int_{\mathbb{S}^{1}}\left(2 v^{-2}-a\right) w^{2} \mathrm{~d} \theta \sigma \mathrm{d} y+\left(\frac{1}{8}-a\right) \int_{\beta y^{2} \geq 20} \int_{\mathbb{S}^{1}} w^{2} \mathrm{~d} \theta \sigma \mathrm{d} y
$$

and

$$
\left|I_{3}\right| \leq C \beta^{2} \int_{\mathbb{R}} \int_{\mathbb{S}^{1}}\left(|w|+\beta\left|\partial_{y}^{3} v\right|+v^{-1}\left|\partial_{y}^{2} \partial_{\theta} v\right|+v^{-2}\left|\partial_{y} \partial_{\theta}^{2} v\right|+v^{-3}\left|\partial_{\theta}^{3} v\right|\right) \mathrm{d} \theta \sigma \mathrm{d} y .
$$

Using Cauchy-Schwarz, the latter estimate becomes

$$
\left|I_{3}\right| \leq \rho \beta^{2}\left(\Omega_{1,1}^{\frac{1}{2}}+\beta \Omega_{3,0}^{\frac{1}{2}}+\Omega_{2,1}^{\frac{1}{2}}+\Omega_{1,2}^{\frac{1}{2}}+\Omega_{0,3}^{\frac{1}{2}}\right) .
$$

To complete the proof, we will estimate $I_{1}:=I_{1,1}+I_{1,2}$, where

$$
\begin{aligned}
& I_{1,1}:=\int_{\mathbb{R}} \int_{\mathbb{S}^{1}} w\left(F_{1} \partial_{y}^{2} w+v^{-2} F_{2} \partial_{\theta}^{2} w+v^{-1} F_{3} \partial_{y} \partial_{\theta} w\right) \mathrm{d} \theta \sigma \mathrm{d} y, \\
& I_{1,2}:=\int_{\mathbb{R}} \int_{\mathbb{S}^{1}} w\left(v^{-2} F_{4} \partial_{\theta} w-a y \partial_{y} w\right) \mathrm{d} \theta \sigma \mathrm{d} y .
\end{aligned}
$$

Because $w$ and $\sigma$ are even functions of $y$, one may integrate by parts in $y$ as well as $\theta$, whereupon applying Cauchy-Schwarz pointwise (as in Corollary 3.3) shows that $I_{1,1} \leq I_{1,3}+I_{1,4}$, where

$$
\begin{aligned}
I_{1,3} & :=-\int_{\mathbb{R}} \int_{\mathbb{S}^{1}} \frac{\left(\partial_{y} w\right)^{2}+v^{-2}\left(\partial_{\theta} w\right)^{2}}{1+p^{2}+q^{2}} \mathrm{~d} \theta \sigma \mathrm{d} y, \\
I_{1,4} & :=\frac{1}{2} \int_{\mathbb{R}} \int_{\mathbb{S}^{1}} w^{2}\left[\sigma^{-1} \partial_{y}^{2}\left(\sigma F_{1}\right)+\partial_{\theta}^{2}\left(v^{-2} F_{2}\right)+\sigma^{-1} \partial_{y} \partial_{\theta}\left(\sigma v^{-1} F_{3}\right)\right] \mathrm{d} \theta \sigma \mathrm{d} y .
\end{aligned}
$$

One estimates $\left(\partial_{y} w\right)^{2} \geq v_{2,1,1}^{2}-C \beta w^{2}$ and $v^{-2}\left(\partial_{\theta} w\right)^{2} \geq v_{1,2,2}^{2}-C \beta^{3} w^{2}$ using Theorem 4.2 and Condition [C1i]. Using those facts and Condition [C0i], one has 
$p^{2}+q^{2} \leq C$ everywhere and $p^{2}+q^{2} \leq \varepsilon$ in the inner region. Hence

$$
\begin{aligned}
I_{1,3} \leq & -\varepsilon\left(\Omega_{2,1}+\Omega_{1,2}\right)+C \beta \Omega_{1,1} \\
& -4(1-2 \varepsilon)\left(\frac{1-\delta}{1+\delta}\right)^{2} \int_{\beta y^{2} \leq 20} \int_{\mathbb{S}^{1}} v^{-2} w^{2} \mathrm{~d} \theta \sigma \mathrm{d} y .
\end{aligned}
$$

To get the final term, we applied Condition [Cr] to the decomposition $v=v_{1}+v_{2}$ introduced in definition (4.2) and then exploited the key idea behind Remark 4.3. Then combining Theorem 4.2 and Conditions [C1i] and [C2] with estimates (6.2)(6.5) and (6.7) lets us control the terms in $I_{1,4}$ using

$$
\begin{aligned}
\sigma^{-1}\left|\partial_{y}^{2}\left(\sigma F_{1}\right)\right| & \leq C\left(\left|\partial_{y}^{3} v\right|+v^{-1}\left|\partial_{y}^{2} \partial_{\theta} v\right|+\beta^{\frac{6}{5}}+\beta^{\frac{3}{5}} \Sigma^{-\frac{1}{2}}+\Sigma^{-1}\right), \\
\left|\partial_{\theta}^{2}\left(v^{-2} F_{2}\right)\right| & \leq C\left(v^{-2}\left|\partial_{y} \partial_{\theta}^{2} v\right|+v^{-3}\left|\partial_{\theta}^{3} v\right|+\beta^{\frac{3}{2}}\right), \\
\sigma^{-1}\left|\partial_{y} \partial_{\theta}\left(\sigma v^{-1} F_{3}\right)\right| & \leq C\left(v^{-1}\left|\partial_{y}^{2} \partial_{\theta} v\right|+v^{-2}\left|\partial_{y} \partial_{\theta}^{2} v\right|+\beta^{\frac{3}{2}}+\beta^{\frac{3}{2}} \Sigma^{-\frac{1}{2}}\right) .
\end{aligned}
$$

Notice that here is where one needs $\Sigma$ to be sufficiently large. To control $I_{1,2}$, one integrates by parts in $\theta$, combining Conditions [C1] and [C1i] with estimate (6.4) to obtain

$$
\left|\int_{\mathbb{R}} \int_{\mathbb{S}^{1}} v^{-2} F_{4}\left(w \partial_{\theta} w\right) \mathrm{d} \theta \sigma \mathrm{d} y\right| \leq C \beta^{\frac{3}{2}} \Omega_{1,1} .
$$

Finally, one uses the fact that $y \partial_{y} \sigma \leq 0$ to get

$$
-a \int_{\mathbb{R}} \int_{\mathbb{S} 1} y w \partial_{y} w \mathrm{~d} \theta \sigma \mathrm{d} y \leq \frac{a}{2} \Omega_{1,1} .
$$

The conclusion of the lemma follows by collecting the estimates above, using the consequence of Condition [C2] that $|w| \lesssim \beta^{\frac{3}{2}}$ pointwise. By Condition [Ca], the coefficient multiplying $\Omega_{1,1}$ can be chosen to be less than $\frac{1}{8}-\frac{a}{2} \leq-\frac{1}{8}+(2 \kappa)^{-1}$.

Lemma 6.6. There exist constants $\varepsilon>0$ and $\rho=\rho(\Sigma)>0$, with $\rho(\Sigma) \searrow 0$ as $\Sigma \rightarrow \infty$, such that

$$
\frac{d}{d \tau} \Omega_{0,2} \leq-\varepsilon\left(\Omega_{0,2}+\Omega_{1,2}+\Omega_{0,3}\right)+\rho \beta^{2}\left(\beta^{\frac{1}{10}} \Omega_{0,2}^{\frac{1}{2}}+\beta \Omega_{2,1}^{\frac{1}{2}}+\Omega_{1,2}^{\frac{1}{2}}+\Omega_{0,3}^{\frac{1}{2}}\right) .
$$

Proof. Because Remark 4.3 applies to $v_{0,2,2} \equiv v^{-2} \partial_{\theta}^{2} v$, the proof is virtually identical, mutatis mutandis to that of Lemma 6.5, when one takes as input the estimates in Lemma 6.3. The final observation is that the coefficient multiplying $\Omega_{0,2}$ can be chosen less than $\frac{3}{16}-\frac{a}{2} \leq-\frac{1}{16}+(2 \kappa)^{-1}$. We omit further details.

Lemma 6.7. There exist constants $\varepsilon>0$ and $\rho=\rho(\Sigma)>0$, with $\rho(\Sigma) \searrow 0$ as $\Sigma \rightarrow \infty$, such that

$$
\frac{d}{d \tau} \Omega_{2,0} \leq-\varepsilon\left(\Omega_{2,0}+\Omega_{3,0}+\Omega_{2,1}\right)+\rho\left[\beta \Omega_{2,0}^{\frac{1}{2}}+\beta^{\frac{1}{2}} \Omega_{3,0}^{\frac{1}{2}}+\beta^{\frac{11}{10}}\left(\Omega_{2,1}^{\frac{1}{2}}+\Omega_{1,2}^{\frac{1}{2}}\right)\right] .
$$

Proof. The argument here contains two key differences from that used above. The estimate for $I_{2}$ in Lemma 6.5 is replaced by

$$
I_{2} \leq\left(\frac{1}{16}-a\right) \Omega_{2,0}+2 I_{2,2}
$$


where

$$
\begin{aligned}
I_{2,2} & :=\int_{\mathbb{R}} \int_{\mathbb{S}^{1}} v^{-2} w^{2} \mathrm{~d} \theta \sigma \mathrm{d} y \\
& =\int_{\mathbb{R}} \int_{\mathbb{S}^{1}}\left(\partial_{y} v\right)\left[2 v^{-3}\left(\partial_{y}^{2} v\right)\left(\partial_{y} v\right)-v^{-2}\left(\partial_{y}^{2} v\right) \sigma^{-1} \partial_{y} \sigma-v^{-2} \partial_{y}^{3} v\right] \mathrm{d} \theta \sigma \mathrm{d} y .
\end{aligned}
$$

Note that $\sigma^{-1}\left|\partial_{y} \sigma\right|=\mathcal{O}\left(\langle y\rangle^{-1}\right)$ as $|y| \rightarrow \infty$. Hence one may apply Condition [Cg] and estimate (6.7) to see that

$$
I_{2,2} \leq \rho\left(\beta \Omega_{2,0}^{\frac{1}{2}}+\beta^{\frac{1}{2}} \Omega_{3,0}^{\frac{1}{2}}\right) .
$$

Remark 4.3 does not apply to the function $\partial_{y}^{2} v$, so one gets only a weaker estimate for $I_{1,3}$, namely

$$
I_{1,3} \leq-\varepsilon\left(\Omega_{3,0}+\Omega_{2,1}\right)+\rho\left(\beta^{2} \Omega_{3,0}^{\frac{1}{2}}+\beta^{3} \Omega_{2,1}^{\frac{1}{2}}\right) .
$$

The remainder of the proof goes through as in Lemma 6.5, using the pointwise estimates derived in Lemma 6.1. Here the coefficient multiplying $\Omega_{2,0}$ can be chosen less than $\frac{1}{16}-\frac{a}{2} \leq-\frac{3}{16}+(2 \kappa)^{-1}$.

We use a modified technique to derive differential inequalities for $\Omega_{m, n}$ with $3 \leq m+n \leq 5$. This technique applies integration by parts to the nonlinear commutators, using important but tedious estimates derived in Appendix D. In the remainder of this subsection, we bound the functionals of order $m+n=3$. We treat the cases $4 \leq m+n \leq 5$ in Section 6.3 below.

Lemma 6.8. There exist constants $0<\varepsilon<C<\infty$ and $\rho=\rho(\Sigma)>0$, with $\rho(\Sigma) \searrow 0$ as $\Sigma \rightarrow \infty$, such that for all $m+n=3$ with $n \geq 1$, one has

$$
\begin{aligned}
\frac{d}{d \tau} \Omega_{m, n} \leq & -\varepsilon\left(\Omega_{m, n}+\Omega_{m+1, n}+\Omega_{m, n+1}\right)+C \beta^{\frac{3}{5}}\left(\Omega_{2,1}+\Omega_{1,2}+\Omega_{0,3}\right) \\
& +\rho \beta^{2}\left[\Omega_{m, n}^{\frac{1}{2}}+\beta \Omega_{4,0}^{\frac{1}{2}}+\Omega_{3,1}^{\frac{1}{2}}+\Omega_{2,2}^{\frac{1}{2}}+\Omega_{1,3}^{\frac{1}{2}}+\Omega_{0,4}^{\frac{1}{2}}\right] .
\end{aligned}
$$

Proof. Let $w:=v_{m, n, n} \equiv v^{-n} \partial_{y}^{m} \partial_{\theta}^{n} v$. Then using dominated convergence and Lemma 3.1, one obtains $\frac{1}{2} \frac{d}{d \tau} \Omega_{m, n}=I_{1}+I_{2}+I_{3}$, with terms $I_{j}$ defined below. We closely follow Lemma 6.5 in estimating the first two terms, indicating why the method used there applies here. We use a somewhat different method to estimate the final term $I_{3}$. For the first term, integration by parts yields

$$
\begin{aligned}
I_{1} & :=\int_{\mathbb{R}} \int_{\mathbb{S}^{1}} w\left(F_{1} \partial_{y}^{2} w+v^{-2} F_{2} \partial_{\theta}^{2} w+v^{-1} F_{3} \partial_{y} \partial_{\theta} w+v^{-2} F_{4} \partial_{\theta} w-a y \partial_{y} w\right) \mathrm{d} \theta \sigma \mathrm{d} y \\
& \leq-\varepsilon\left(\Omega_{m+1, n}+\Omega_{m, n+1}\right)+\left(\frac{a}{2}+\varepsilon\right) \Omega_{m, n}-(4-\varepsilon) \int_{\beta y^{2} \leq 20} \int_{\mathbb{S}^{1}} v^{-2} w^{2} \mathrm{~d} \theta \sigma \mathrm{d} y .
\end{aligned}
$$

Here we followed Lemma 6.5, estimating

$$
\left(\partial_{y} w\right)^{2}+\left(\partial_{\theta} w\right)^{2} \geq v_{m+1, n, n}^{2}+v_{m, n+1, n+1}^{2}-C \beta w^{2},
$$

using Condition [C3] to bound the third-order derivatives in display (6.8) by $C \beta$, and again exploiting the idea behind Remark 4.3 to get the useful integral over the 
inner region. By Lemma 3.1 and estimate (4.3), one has

$$
\begin{aligned}
I_{2} & :=\int_{\mathbb{R}} \int_{\mathbb{S}^{1}}\left[(n+1) v^{-2}-2 a\right] w^{2} \mathrm{~d} \theta \sigma \mathrm{d} y \\
& \leq\left(\frac{1}{4}-\frac{3 a}{2}\right) \Omega_{m, n}+\left(4-\frac{a}{2}\right) \int_{\beta y^{2} \leq 20} \int_{\mathbb{S}^{1}} v^{-2} w^{2} \mathrm{~d} \theta \sigma \mathrm{d} y .
\end{aligned}
$$

In Appendix D, we estimate

$$
I_{3}:=\int_{\mathbb{R}} \int_{\mathbb{S}^{1}}\left(\sum_{\ell=0}^{5} E_{m, n, n, \ell}\right) w \mathrm{~d} \theta \sigma \mathrm{d} y
$$

using integration by parts. By Lemma D.1 there, one has

$\left|I_{3}\right| \leq \rho \beta^{2}\left[\Omega_{m, n}^{\frac{1}{2}}+\beta \Omega_{4,0}^{\frac{1}{2}}+\Omega_{3,1}^{\frac{1}{2}}+\Omega_{2,2}^{\frac{1}{2}}+\Omega_{1,3}^{\frac{1}{2}}+\Omega_{0,4}^{\frac{1}{2}}\right]+C \beta^{\frac{3}{5}}\left(\Omega_{2,1}+\Omega_{1,2}+\Omega_{0,3}\right)$.

Collecting these estimates while recalling Condition $[\mathrm{Ca}]$ completes the proof.

Lemma 6.9. There exist constants $0<\varepsilon<C<\infty$ and $\rho=\rho(\Sigma)>0$, with $\rho(\Sigma) \searrow 0$ as $\Sigma \rightarrow \infty$, such that

$$
\begin{aligned}
\frac{d}{d \tau} \Omega_{3,0} \leq & -\varepsilon\left(\Omega_{3,0}+\Omega_{4,0}+\Omega_{3,1}\right)+\rho \beta^{\frac{3}{2}}\left[\Omega_{3,0}^{\frac{1}{2}}+\beta^{\frac{1}{10}} \Omega_{4,0}^{\frac{1}{2}}+\Omega_{3,1}^{\frac{1}{2}}+\Omega_{2,2}^{\frac{1}{2}}\right] \\
& +C \beta\left(\sum_{i+j=3} \Omega_{i, j}\right)+C \beta^{\frac{1}{2}} \Omega_{3,0}^{\frac{1}{2}} \Omega_{2,0}^{\frac{1}{2}} .
\end{aligned}
$$

Proof. We again write $\frac{1}{2} \frac{d}{d \tau} \Omega_{3,0}=I_{1}+I_{2}+I_{3}$ as in the proof of Lemma 6.8. The only differences from the proof there are (I) we use Lemma D.2 to estimate $I_{3} \equiv E_{3,0}$, and (II) we cannot apply Remark 4.3 to extract a useful integral over the inner region from $I_{1}$. However, that integral is not needed. Indeed, by Conditions [C0i] and $[\mathrm{Ca}]$, one has $v^{-2}-\frac{4 a}{3} \leq \frac{50}{81}-\frac{2}{3}+\varepsilon<0$. (Compare Remark 3.2.) Therefore,

$$
I_{2}:=\int_{\mathbb{R}} \int_{\mathbb{S}^{1}}\left(v^{-2}-2 a\right)\left(v_{3,0,0}\right)^{2} \mathrm{~d} \theta \sigma \mathrm{d} y \leq-\frac{2 a}{3} \Omega_{3,0} .
$$

The remainder of the proof exactly follows that of Lemma 6.8. (Note that we deal with the term $C \beta^{\frac{1}{2}} \Omega_{3,0}^{\frac{1}{2}} \Omega_{2,0}^{\frac{1}{2}}$ in the proof of Proposition 6.10 below).

We are now ready to bound the Lyapunov functionals $\Omega_{m, n}$ with $2 \leq m+n \leq 3$.

Proposition 6.10. Suppose that a solution $v=v(y, \theta, \tau)$ of equation (2.5) satisfies Assumption [A1] at $\tau=0$, as well as [Ca], [CO]-[C3], [COi]-[C1i], [Cg], [Cr], and [Cs] for $\tau \in\left[0, \tau_{1}\right]$. Then for the same time interval, one has

$$
\beta^{2} \Omega_{2,0}+\Omega_{1,1}+\Omega_{0,2}+\beta \Omega_{3,0}+\Omega_{2,1}+\Omega_{1,2}+\Omega_{0,3} \lesssim \beta^{4} .
$$

Proof. For $R>0$ to be chosen, define

$$
\Upsilon:=R\left(\beta^{2} \Omega_{2,0}+\Omega_{1,1}+\Omega_{0,2}\right)+\beta \Omega_{3,0}+\Omega_{2,1}+\Omega_{1,2}+\Omega_{0,3},
$$

noting the $\beta$-weights on the first and fourth terms. Observe that $\frac{d}{d \tau} \beta<0$. Thus by Lemmas 6.7 and 6.9 , respectively, there exist $\varepsilon_{2}, \varepsilon_{3}>0$ such that

$$
\frac{d}{d \tau}\left(\beta^{2} \Omega_{2,0}\right) \leq-\varepsilon_{2}\left(\beta^{2} \Omega_{2,0}\right)+\rho \beta^{2}\left[\left(\beta^{2} \Omega_{2,0}\right)^{\frac{1}{2}}+\left(\beta \Omega_{3,0}\right)^{\frac{1}{2}}+\Omega_{2,1}^{\frac{1}{2}}+\Omega_{1,2}^{\frac{1}{2}}\right]
$$


and

$$
\begin{aligned}
\frac{d}{d \tau}\left(\beta \Omega_{3,0}\right) \leq & -\varepsilon_{3}\left(\beta \Omega_{3,0}\right)+\rho \beta^{2}\left[\left(\beta \Omega_{3,0}\right)^{\frac{1}{2}}+\beta^{\frac{3}{5}} \Omega_{4,0}^{\frac{1}{2}}+\Omega_{3,1}^{\frac{1}{2}}+\Omega_{2,2}^{\frac{1}{2}}\right] \\
& +C \beta\left[\left(\beta \Omega_{3,0}\right)+\beta\left(\Omega_{2,1}+\Omega_{1,2}+\Omega_{0,3}\right)\right] \\
& +\left\{\frac{\varepsilon_{3}}{2}\left(\beta \Omega_{3,0}\right)+\frac{C^{2}}{2 \varepsilon_{3}}\left(\beta^{2} \Omega_{2,0}\right)\right\},
\end{aligned}
$$

where one applies weighted Cauchy-Schwarz to $C\left(\beta^{2} \Omega_{2,0}\right)^{\frac{1}{2}}\left(\beta \Omega_{3,0}\right)^{\frac{1}{2}}$ to obtain the terms in braces.

Set $R=\frac{C^{2}}{\varepsilon_{2} \varepsilon_{3}}$. Then collecting the remaining estimates in Lemmata $6.5-6.8$ and applying Cauchy-Schwarz yet again proves that there exist $\varepsilon_{0}>0$ and $C_{0}<\infty$, both independent of $R$, such that

$$
\frac{d}{d \tau} \Upsilon \leq-\varepsilon_{0} \Upsilon+C_{0}\left(R \rho \beta^{2} \Upsilon^{\frac{1}{2}}+\rho^{2} \beta^{4}\right)
$$

whence it follows easily that $\Upsilon \lesssim \beta^{4}$.

6.3. Lyapunov functionals of fourth and fifth order. In this subsection, we derive differential inequalities satisfied by the $\Omega_{m, n}$ with $4 \leq m+n \leq 5$. As noted above, our estimates for them do not need to be sharp, which somewhat reduces the technical work needed to bound the relevant nonlinear terms. This work, which is done in Lemmata D.3-D.7 of Appendix D, is also substantially reduced by the availability of Proposition 6.10.

Lemma 6.11. There exist $0<\varepsilon<C<\infty$ such that whenever $m+n=4$,

$$
\begin{aligned}
\frac{d}{d \tau} \Omega_{m, n} \leq & -\varepsilon\left(\Omega_{m, n}+\Omega_{m+1, n}+\Omega_{m, n+1}\right)+C \beta^{\frac{1}{2}}\left(\sum_{4 \leq i+j \leq 5} \Omega_{i, j}\right) \\
& +C \beta^{r}\left(\sum_{4 \leq i+j \leq 5} \Omega_{i, j}^{\frac{1}{2}}\right),
\end{aligned}
$$

where $r=\frac{8}{5}$ if $n=0$ and $r=2$ otherwise.

Proof. Following the proof of Lemma 6.8, the result follows easily when one observes that

$$
\begin{aligned}
\int_{\mathbb{R}} \int_{\mathbb{S}^{1}}\left[(n+1) v^{-2}-(\right. & +n-1) a] v_{m, n, n}^{2} \mathrm{~d} \theta \sigma \mathrm{d} y \\
\leq & \left(\frac{3}{8}-\frac{5 a}{2}\right) \Omega_{m, n}+\left(4-\frac{a}{2}\right) \int_{\beta y^{2} \leq 20} \int_{\mathbb{S}^{1}} v^{-2} w^{2} \mathrm{~d} \theta \sigma \mathrm{d} y
\end{aligned}
$$

and applies the conclusion of Lemma D.6 from Appendix D.

Lemma 6.12. There exist $0<\varepsilon<C<\infty$ such that whenever $m+n=5$,

$$
\begin{aligned}
\frac{d}{d \tau} \Omega_{m, n} \leq & -\varepsilon\left(\Omega_{m, n}+\Omega_{m+1, n}+\Omega_{m, n+1}\right)+C \beta^{\frac{1}{2}}\left(\sum_{4 \leq i+j \leq 6} \Omega_{i, j}\right) \\
& +C \beta^{\frac{21}{10}}\left(\sum_{5 \leq i+j \leq 6} \Omega_{i, j}^{\frac{1}{2}}\right) .
\end{aligned}
$$


Proof. The proof exactly parallels that of Lemma 6.8 when one observes that

$$
\int_{\mathbb{R}} \int_{\mathbb{S}^{1}}\left[v^{-2}-(m-1) a\right] v_{m, 0,0}^{2} \mathrm{~d} \theta \sigma \mathrm{d} y \leq-\frac{5 a}{3} \Omega_{m, 0}
$$

and applies Lemma D.7 from Appendix D.

\subsection{Estimates of second- and third-order derivatives.}

Proof of Theorem 4.5. As indicated above, the proof is in three steps.

(STEP I) This was accomplished in Proposition 6.10, where we proved that

$$
\beta^{2} \Omega_{2,0}+\Omega_{1,1}+\Omega_{0,2}+\beta \Omega_{3,0}+\Omega_{2,1}+\Omega_{1,2}+\Omega_{0,3} \lesssim \beta^{4} .
$$

(STEP II) Define

$$
\Upsilon:=\beta^{\frac{4}{5}} \Omega_{4,0}+\Omega_{3,1}+\Omega_{2,2}+\Omega_{1,3}+\Omega_{0,4}+\sum_{m+n=5} \Omega_{m, n},
$$

noting the $\beta$-weight imposed on the first term. By Lemmas 6.11-6.12 and CauchySchwarz, there exist $0<\varepsilon<C<\infty$ such that

$$
\frac{d}{d \tau} \Upsilon \leq-\varepsilon \Upsilon+C\left(\beta^{2} \Upsilon^{\frac{1}{2}}+\beta^{4}\right) .
$$

Assumption [A6] bounds $\Upsilon$ at $\tau=0$. It follows that $\Upsilon \lesssim \beta^{4}$, namely that

$$
\beta^{\frac{4}{5}} \Omega_{4,0}+\Omega_{3,1}+\Omega_{2,2}+\Omega_{1,3}+\Omega_{0,4}+\sum_{m+n=5} \Omega_{m, n} \lesssim \beta^{4} .
$$

(STEP III) Now that we have $L_{\sigma}^{2}$ bounds on derivatives of orders two through five, Sobolev embedding gives pointwise bounds on derivatives of orders two through three. Because of the weighted norm $\|\cdot\|_{\sigma}$ these pointwise bounds are not uniform in $y$. Using the facts that $\sigma \sim\langle y\rangle^{-\frac{6}{5}}$ as $|y| \rightarrow \infty$ and that $|y| \lesssim \beta^{-\frac{1}{2}}$ in the inner region, one obtains

$$
\beta^{2}\left(\partial_{y}^{2} v\right)^{2}+v^{-2}\left(\partial_{y} \partial_{\theta} v\right)^{2}+v^{-4}\left(\partial_{\theta}^{2} v\right)^{2} \lesssim \beta^{4-\frac{1}{20}-\frac{3}{5}}
$$

and

$$
\beta\left(\partial_{y}^{3} v\right)^{2}+v^{-2}\left(\partial_{y}^{2} \partial_{\theta} v\right)^{2}+v^{-4}\left(\partial_{y} \partial_{\theta}^{2} v\right)^{2}+v^{-6}\left(\partial_{\theta}^{3} v\right)^{2} \lesssim \beta^{4-\frac{1}{20}-\frac{3}{5}}
$$

in the inner region. These inequalities are equivalent to estimates (4.8)-(4.9).

\section{Estimates in the OUTER REgion}

7.1. Second-order decay estimates. As noted above, it is easy in the outer region to "close the loop" by estimating second-order derivatives without needing assumptions on higher derivatives.

Proof of estimate (4.10). By Lemmata 6.1-6.3 and Cauchy-Schwarz, there exist constants $C_{1}, C_{2}, C_{3}$ such that in the outer region, one has

$$
\begin{aligned}
\partial_{\tau}\left|\partial_{y}^{2} v\right|^{2} & \leq A_{v}\left(\left|\partial_{y}^{2} v\right|^{2}\right)-\frac{5}{8}\left|\partial_{y}^{2} v\right|^{2}-\varepsilon\left(\left|\partial_{y}^{3} v\right|^{2}+v^{-2}\left|\partial_{y}^{2} \partial_{\theta} v\right|^{2}\right) \\
& +C_{1}\left(\beta^{2}+\beta^{\frac{11}{10}} v^{-2}\left|\partial_{y} \partial_{\theta}^{2} v\right|\right)
\end{aligned}
$$

and

$$
\begin{aligned}
\partial_{\tau}\left(v^{-2}\left|\partial_{y} \partial_{\theta} v\right|^{2}\right) & \leq A_{v}\left(v^{-2}\left|\partial_{y} \partial_{\theta} v\right|^{2}\right)-\frac{1}{2} v^{-2}\left|\partial_{y} \partial_{\theta} v\right|^{2}-\varepsilon\left(v^{-2}\left|\partial_{y}^{2} \partial_{\theta} v\right|^{2}+v^{-4}\left|\partial_{y} \partial_{\theta}^{2} v\right|^{2}\right) \\
& +C_{2}\left(\beta^{4}+\beta^{3}\left|\partial_{y}^{3} v\right|+\beta^{2} v^{-3}\left|\partial_{\theta}^{3} v\right|\right)
\end{aligned}
$$


and

$$
\begin{aligned}
\partial_{\tau}\left(v^{-4}\left|\partial_{\theta}^{2} v\right|^{2}\right) & \leq A_{v}\left(v^{-4}\left|\partial_{\theta}^{2} v\right|^{2}\right)-\frac{3}{8} v^{-4}\left|\partial_{\theta}^{2} v\right|^{2}-\varepsilon\left(v^{-4}\left|\partial_{y} \partial_{\theta}^{2} v\right|^{2}+v^{-6}\left|\partial_{\theta}^{3} v\right|^{2}\right) \\
& +C_{3}\left(\beta^{4}+\beta^{3} v^{-1}\left|\partial_{y}^{2} \partial_{\theta} v\right|\right) .
\end{aligned}
$$

Define

$$
\Upsilon:=\beta^{2}\left|\partial_{y}^{2} v\right|^{2}+v^{-2}\left|\partial_{y} \partial_{\theta} v\right|^{2}+v^{-4}\left|\partial_{\theta}^{2} v\right|^{2}
$$

noting the $\beta$-weight imposed on the first term. Then because $\frac{d}{d \tau} \beta<0$, there exists $C_{0}<\infty$ such that

where

$$
\partial_{\tau} \Upsilon \leq A_{v}(\Upsilon)-\frac{3}{8} \Upsilon+C_{0} \beta^{4}+E
$$

$$
\begin{aligned}
E & :=\left(C_{2} \beta^{3}\left|\partial_{y}^{3} v\right|-\varepsilon \beta\left|\partial_{y}^{3} v\right|^{2}\right)+\left(C_{3} \beta^{3} v^{-1}\left|\partial_{y}^{2} \partial_{\theta} v\right|-\varepsilon v^{-2}\left|\partial_{y}^{2} \partial_{\theta} v\right|^{2}\right) \\
& +\left(C_{1} \beta^{\frac{31}{10}} v^{-2}\left|\partial_{y} \partial_{\theta}^{2} v\right|-\varepsilon v^{-4}\left|\partial_{y} \partial_{\theta}^{2} v\right|^{2}\right)+\left(C_{2} \beta^{2} v^{-3}\left|\partial_{\theta}^{3} v\right|-\varepsilon v^{-6}\left|\partial_{\theta}^{3} v\right|^{2}\right)
\end{aligned}
$$

Cauchy-Schwarz proves that $E \lesssim \beta^{4}$. Hence there exists $C<\infty$ such that

$$
\partial_{\tau}\left(\Upsilon-\Gamma \beta^{\frac{33}{10}}\right) \leq A_{v}\left(\Upsilon-\Gamma \beta^{\frac{33}{10}}\right)-\frac{3}{8}\left(\Upsilon-\Gamma \beta^{\frac{33}{10}}\right)+\left[\frac{33}{10} \beta+\frac{C}{\Gamma} \beta^{\frac{7}{10}}-\frac{3}{8}\right] \Gamma \beta^{\frac{33}{10}} .
$$

Choosing $\kappa$ and $\Gamma$ sufficiently large ensures both that the quantity in brackets above is negative, and that $\Upsilon \leq \Gamma \beta^{\frac{33}{10}}$ at $\tau=0$. By Theorem 4.5, one has $\Upsilon \leq \Gamma \beta^{\frac{33}{10}}$ on the rest of the parabolic boundary. Hence the result follows from the maximum principle in the form of Proposition C.1.

7.2. Third-order decay estimates. In this subsection, we complete the proof of Theorem 4.6 by establishing estimate (4.11) in the outer region.

As we did in the previous section, we begin by deriving differential inequalities satisfied by the squares of the quantities that appear in (4.11). These estimates do not have to be sharp, because we only need relatively weak third-order estimates for our application in Section 8. To keep the notation from becoming too cumbersome, we consistently write $\left(v_{m, n, k}\right)$ for $\left(v^{-k} \partial_{y}^{m} \partial_{\theta}^{n} v\right)$ in the remainder of this section.

Lemma 7.1. There exist $0<\varepsilon<C<\infty$ such that in the outer region,

$$
\begin{aligned}
\partial_{\tau}\left(v_{3,0,0}\right)^{2} \leq & A_{v}\left(\left(v_{3,0,0}\right)^{2}\right)-\frac{7}{4}\left(v_{3,0,0}\right)^{2}-\varepsilon\left[\left(v_{4,0,0}\right)^{2}+\left(v_{3,1,1}\right)^{2}\right] \\
& +C\left(\beta^{3}+\beta^{\frac{6}{5}}\left|\left(v_{3,0,0}\right)\right|+\beta^{\frac{3}{2}}\left|\left(v_{2,2,2}\right)\right|+\beta \sum_{m+n=3} v_{m, n, n}^{2}\right) .
\end{aligned}
$$

Proof. Following the proof of Lemma 6.1, one applies Condition [Ca] and estimates (4.3), (4.4), and (4.6) to equations (3.4)-(3.5) to bound the linear terms above. So it suffices to derive a pointwise bound for $\left|\left(v_{3,0,0}\right) \sum_{\ell=1}^{5} E_{3,0,0, \ell}\right|$.

For this, we can use some but not all of the estimates derived in Lemma D.2. Four terms there were estimated using integration by parts. Here, we instead combine the pointwise bounds derived in Lemma D.4 with estimates (6.1)-(6.3) to obtain the bounds

$$
\left|\left(v_{3,0,0}\right)\left(\partial_{y}^{3} F_{1}\right)\left(v_{2,0,0}\right)\right| \lesssim \beta^{\frac{7}{10}}\left|\left(v_{3,0,0}\right)\right|\left(\beta^{\frac{8}{5}}+\left|\left(v_{4,0,0}\right)\right|+\left|\left(v_{3,1,1}\right)\right|\right)
$$

and

$$
\left|\left(v_{3,0,0}\right)\left(\partial_{y}^{3} F_{2}\right)\left(v_{0,2,2}\right)\right| \lesssim \beta^{\frac{3}{2}}\left|\left(v_{3,0,0}\right)\right|\left(\beta^{\frac{8}{5}}+\left|\left(v_{4,0,0}\right)\right|+\left|\left(v_{3,1,1}\right)\right|\right)
$$


with similar estimates holding for the contributions from $F_{3}$ and $F_{4}$. Again using estimates (6.1)-(6.3) instead of the method used in Lemma D.2, we obtain the critical bound

$$
\left|\left(v_{3,0,0}\right) E_{3,0,0,5}\right| \lesssim\left|\left(v_{3,0,0}\right)\right|\left|\left(v_{2,0,0}\right)\left(v_{1,0,1}\right)+\left(v_{1,0,1}\right)^{3}\right| \lesssim \beta^{\frac{6}{5}}\left|\left(v_{3,0,0}\right)\right| .
$$

The result follows using Cauchy-Schwarz and our assumption $\left|\left(v_{3,0,0}\right)\right| \lesssim \beta$.

Lemma 7.2. There exist $0<\varepsilon<C<\infty$ such that for $m+n=3$ with $n \geq 1$, one estimates in the outer region that

$$
\begin{aligned}
\partial_{\tau}\left(v_{m, n, n}\right)^{2} \leq & A_{v}\left(\left(v_{m, n, n}\right)^{2}\right)-\frac{5}{4}\left(v_{m, n, n}\right)^{2}-\varepsilon\left[\left(v_{m+1, n, n}\right)^{2}+\left(v_{m, n+1, n+1}\right)^{2}\right] \\
& +C \beta^{2}\left(\left|v_{m, n, n}\right|+\beta\left|v_{4,0,0}\right|+\left|v_{3,1,1}\right|+\left|v_{2,2,2}\right|+\left|v_{1,3,3}\right|+\left|v_{0,4,4}\right|\right) \\
& +C \beta^{\frac{3}{5}}\left[\left(v_{2,1,1}\right)^{2}+\left(v_{1,2,2}\right)^{2}+\left(v_{0,3,3}\right)^{2}\right] .
\end{aligned}
$$

Proof. As in the proof of Lemma 7.1, we may apply Condition [Ca] and estimates (4.3), (4.4), and (4.6) to equations (3.4)-(3.5) in order to bound the linear terms above.

To derive a pointwise bound for $\left|\left(v_{m, n, n}\right) \sum_{\ell=0}^{5} E_{m, n, n, \ell}\right|$, we use some but not all of the estimates derived in Lemma D.1. To replace the estimates that were obtained there using integration by parts, we again combine the pointwise bounds derived in Lemma D.4 with estimates (6.1)-(6.3), obtaining, for example,

$\left|\left(v_{m, n, n}\right)\left(v^{-n} \partial_{y}^{m} \partial_{\theta}^{n} F_{1}\right)\left(v_{2,0,0}\right)\right| \lesssim \beta^{\frac{7}{10}}\left|\left(v_{m, n, n}\right)\right|\left(\beta^{2}+\left|\left(v_{m+1, n, n}\right)\right|+\left|\left(v_{m, n+1, n+1}\right)\right|\right)$,

with stronger estimates holding for the contributions from $F_{\ell}, \ell=2,3,4$. Here, the term $\left|\left(v_{m, n, n}\right) E_{m, n, n, 5}\right|$ is easy to estimate. Thus the result again follows from Cauchy-Schwarz and our assumption that $\left|\left(v_{m, n, n}\right)\right| \lesssim \beta^{\frac{3}{2}}$.

Now we are ready to prove our third-order decay estimates in the outer region.

Proof of estimate (4.11). Define

$$
\Upsilon:=\beta\left(v_{3,0,0}\right)^{2}+\left(v_{2,1,1}\right)^{2}+\left(v_{1,2,2}\right)^{2}+\left(v_{0,3,3}\right)^{2} .
$$

Then, just as in the proof of estimate (4.10), it follows from Lemmas 7.1-7.2 and Cauchy-Schwarz that

$$
\begin{aligned}
\partial_{\tau} \Upsilon & \leq A_{v}(\Upsilon)-\Upsilon+C_{0}\left(\beta^{\frac{17}{10}} \Upsilon^{\frac{1}{2}}+\beta^{4}\right) \\
& \leq A_{v}(\Upsilon)-\frac{7}{8} \Upsilon+C \beta^{\frac{34}{10}}
\end{aligned}
$$

By hypothesis, one has $\Upsilon \lesssim \beta^{\frac{33}{10}}$ on the parabolic boundary of the outer region. Thus applying the parabolic maximum principle in the form of Proposition C.1, exactly as in the proof of estimate (4.10), shows that $\Upsilon \lesssim \beta^{\frac{33}{10}}$ throughout the outer region for $\tau \in\left[0, \tau_{1}\right]$.

This completes our proof of Theorem 4.6. 
7.3. Third-order smallness estimates. Now we prove Theorem 4.7, whose purpose is to bound $\left|\partial_{y} \partial_{\theta}^{2} v\right|$ and $v^{-1}\left|\partial_{\theta}^{3} v\right|$ for use in Section 8 below. ${ }^{8}$

Define

$$
\Upsilon:=\left(v^{-1} \partial_{\theta}^{3} v\right)^{2}+\left(\partial_{y} \partial_{\theta}^{2} v\right)^{2}+\beta^{-\frac{11}{10}}\left[\left(\partial_{y}^{2} \partial_{\theta} v\right)^{2}+\left(\partial_{y}^{3} v\right)^{2}\right] .
$$

The factor $\beta^{-\frac{11}{10}}$ will be used in estimate (7.3) below. In this section, we prove:

Proposition 7.3. There exists a constant $C$ such that in the outer region $\beta y^{2} \geq 20$,

$$
\partial_{\tau} \Upsilon \leq A_{v} \Upsilon+C \beta^{\frac{11}{10}} .
$$

We claim that Theorem 4.7 is an easy corollary of this result. Indeed, by our Main Assumptions, one has

$$
\Upsilon(\cdot, \cdot, 0) \leq \varepsilon_{0}
$$

for some $\varepsilon_{0} \ll 1$. In the inner region $\beta y^{2} \leq 20$, one combines the consequence $v \leq C_{0}$ of Condition [C0i] with the results of Theorem 4.5 to see that

$$
\left.\Upsilon\right|_{\beta y^{2} \leq 20} \lesssim \beta^{\frac{1}{2}} \text {. }
$$

Together, these estimates control $\Upsilon$ on the parabolic boundary of the outer region. Thus by Proposition 7.3 and the parabolic maximum principle, one has

$$
\begin{aligned}
\Upsilon(\cdot, \cdot, \tau) & \leq \varepsilon_{0}+\beta^{\frac{1}{2}}(\tau)+C \int_{0}^{\tau} \beta^{\frac{11}{10}}(\tau) \mathrm{d} \tau \\
& \lesssim\left(\varepsilon_{0}+\beta_{0}\right)^{\frac{1}{10}},
\end{aligned}
$$

which proves Theorem 4.7.

Proof of Proposition 7.3. We start by studying $\Upsilon_{2}:=\left(\partial_{y} \partial_{\theta}^{2} v\right)^{2}$, since it forces us to define $\Upsilon$ as in (7.1). Apply Corollary 3.3 to obtain

$$
\partial_{\tau} \Upsilon_{2}=A_{v} \Upsilon_{2}+2 v^{-2} \Upsilon_{2}-B_{1,2,0}+2 E_{1,2,0}\left(\partial_{y} \partial_{\theta}^{2} v\right)
$$

For the term $2 v^{-2} \Upsilon_{2}$, we write $v^{-1}\left|\partial_{y} \partial_{\theta}^{2} v\right| \leq\left(v^{-2}\left|\partial_{y} \partial_{\theta}^{2} v\right|\right)^{\frac{1}{2}}\left|\partial_{y} \partial_{\theta}^{2} v\right|^{\frac{1}{2}}$ and employ Theorem 4.6 and the smallness estimate in Condition [C3] to estimate

$$
2 v^{-2}\left|\partial_{y} \partial_{\theta}^{2} v\right|^{2} \leq \beta^{\frac{3}{2}}
$$

In this way, we derive the differential inequality

$$
\partial_{\tau} \Upsilon_{2} \leq A_{v} \Upsilon_{2}-B_{1,2,0}+2 E_{1,2,0}\left(\partial_{y} \partial_{\theta}^{2} v\right)+\beta^{\frac{3}{2}} .
$$

Next we consider the term $E_{1,2,0}\left(\partial_{y} \partial_{\theta}^{2} v\right)$. In the present situation, the difficulties encountered are similar to those surmounted in the proof of Theorem 4.6; we handle these in a similar manner.

We treat the highest-order terms first. Among the many terms that make up $E_{1,2,0}$, some contain a factor (at most one) of $\partial_{y}^{m} \partial_{\theta}^{n} v$ with $m+n=4$. If $(m, n)=(2,2),(1,3)$, this factor is controlled by the favorable term $-B_{1,2,0}$ in inequality $(7.2)$. The difficult cases are $(m, n)=(0,4),(3,1)$. There are only two such terms in $E_{1,2,0}\left(\partial_{y} \partial_{\theta}^{2} v\right)$, namely

$$
\begin{aligned}
D_{1} & :=\left(\partial_{y} \partial_{\theta}^{2} v\right)\left(\partial_{\theta} F_{1}\right)\left(\partial_{y}^{3} \partial_{\theta} v\right) \\
& =\left(\partial_{y} \partial_{\theta}^{2} v\right)\left[\left(\partial_{p} F_{1}\right)\left(\partial_{y} \partial_{\theta} v\right)+\left(\partial_{q} F_{1}\right)\left(\partial_{\theta} q\right)\right]\left(\partial_{y}^{3} \partial_{\theta} v\right)
\end{aligned}
$$

\footnotetext{
${ }^{8}$ Note that Lemma B.2 lets us control $\left|\partial_{y} \partial_{\theta} v\right|$ and $v^{-1}\left|\partial_{\theta}^{k} v\right|$ for $k=1,2$ by bounding $\left|\partial_{y} \partial_{\theta}^{2} v\right|$ and $v^{-1}\left|\partial_{\theta}^{3} v\right|$, respectively.
} 
and

$$
\begin{aligned}
D_{2} & :=\left(\partial_{y} \partial_{\theta}^{2} v\right)\left(\partial_{y} F_{2}\right)\left(v^{-2} \partial_{\theta}^{4} v\right) \\
& =\left(\partial_{y} \partial_{\theta}^{2} v\right)\left[\left(\partial_{p} F_{2}\right)\left(\partial_{y}^{2} v\right)+\left(\partial_{q} F_{2}\right)\left(\partial_{y} q\right)\right]\left(v^{-2} \partial_{\theta}^{4} v\right) .
\end{aligned}
$$

For $D_{1}$, combining Lemma B.2 with the smallness estimate in Condition [C3] shows that the terms in brackets admit the estimate

$$
\left|\left(\partial_{p} F_{1}\right)\left(\partial_{y} \partial_{\theta} v\right)+\left(\partial_{q} F_{1}\right)\left(\partial_{\theta} q\right)\right| \leq \frac{\left(\beta_{0}+\varepsilon_{0}\right)^{\frac{1}{20}}}{1+p^{2}+q^{2}} .
$$

Using Cauchy-Schwarz and the smallness assumption again, one thus obtains

$$
\begin{aligned}
\left|D_{1}\right| & \leq\left(\beta_{0}+\varepsilon_{0}\right)^{\frac{1}{20}}\left[\beta^{\frac{11}{10}}\left|\partial_{y} \partial_{\theta}^{2} v\right|^{2}+\frac{\beta^{-\frac{11}{10}}\left(\partial_{y}^{3} \partial_{\theta} v\right)^{2}}{1+p^{2}+q^{2}}\right] \\
& \leq\left(\beta_{0}+\varepsilon_{0}\right)^{\frac{1}{10}} \beta^{\frac{11}{10}}+\left(\beta_{0}+\varepsilon_{0}\right)^{\frac{1}{20}} \beta^{-\frac{11}{10}} B_{2,1,0},
\end{aligned}
$$

where $\beta^{-\frac{11}{10}} B_{2,1,0}$ appears in the evolution equation for $\beta^{-\frac{11}{10}}\left(\partial_{y}^{2} \partial_{\theta} v\right)^{2}$ below.

For $D_{2}$, we proceed differently. Here, the decay estimate comes from the terms in brackets. By the estimates in Theorem 4.6 and the interpolation Lemma B.2, one has

$$
\left|\left(\partial_{p} F_{2}\right)\left(\partial_{y}^{2} v\right)+\left(\partial_{q} F_{2}\right)\left(\partial_{y} q\right)\right| \lesssim \frac{\left|\partial_{y}^{2} v\right|+\left|\partial_{y} q\right|}{1+p^{2}+q^{2}} \lesssim \frac{\beta^{\frac{13}{20}}}{1+p^{2}+q^{2}}
$$

Hence

$$
\left|D_{2}\right| \leq\left(\beta_{0}+\varepsilon_{0}\right)^{\frac{1}{10}} \beta^{\frac{11}{10}}+\frac{\left(\beta_{0}+\varepsilon_{0}\right)^{\frac{1}{20}}}{1+p^{2}+q^{2}} B_{0,3,1},
$$

where $B_{0,3,1}$ appears in the evolution equation for $v^{-2}\left(\partial_{\theta}^{3} v\right)^{2}$ below.

The remaining terms in inequality (7.2) are estimated by techniques very similar to those employed in the proof of Theorem 4.6. Those methods may be used because we have available the smallness estimates from Condition [C3]. Together with estimate (7.3) and estimate (7.4), we apply these techniques here (omitting further details) to obtain

$$
\partial_{\tau} \Upsilon_{2} \leq A_{v} \Upsilon_{2}-\frac{1}{2} B_{1,2,0}+\frac{B_{0,3,0}+\beta^{-\frac{11}{10}} B_{2,1,0}}{1+p^{2}+q^{2}}+\beta^{\frac{11}{10}}
$$

Now we turn to $\Upsilon_{3}:=\beta^{-\frac{11}{10}}\left(\partial_{y}^{2} \partial_{\theta} v\right)^{2}$. We use Corollary 3.3 to write $\partial_{\tau} \Upsilon_{3}=A_{v} \Upsilon_{3}+2\left[v^{-2}-a+\beta^{\frac{11}{10}}\left(\partial_{\tau} \beta^{-\frac{11}{10}}\right)\right] \Upsilon_{3}-\beta^{-\frac{11}{10}} B_{2,1,0}+2 \beta^{-\frac{11}{10}} E_{2,1,0}\left(\partial_{y}^{2} \partial_{\theta} v\right)$. From this, we use the implication of Theorem 4.6 that $v^{-2}\left(\partial_{y}^{2} \partial_{\theta} v\right)^{2} \lesssim \beta^{\frac{33}{10}}$ and the fact that $\beta^{\frac{11}{10}}\left(\partial_{\tau} \beta^{-\frac{11}{10}}\right)=\frac{11}{10} \beta \ll \frac{1}{10}$ to derive the differential inequality

$$
\partial_{\tau} \Upsilon_{3} \leq A_{v} \Upsilon_{3}-\frac{1}{2} \Upsilon_{3}-\beta^{-\frac{11}{10}} B_{2,1,0}+2 \beta^{-\frac{11}{10}} E_{2,1,0}\left(\partial_{y}^{2} \partial_{\theta} v+\beta^{2}\right) \text {. }
$$

This differs from the corresponding inequality (7.2) for $\partial_{\tau} \Upsilon_{2}$ in by the term $-\frac{1}{2} \Upsilon_{3}$.

As in the derivation of inequality (7.5), there are two critical terms,

$$
W_{1}:=\beta^{-\frac{11}{10}}\left(\partial_{y}^{2} \partial_{\theta} v\right)\left(\partial_{\theta} F_{1}\right)\left(\partial_{y}^{4} v\right)
$$

and

$$
W_{2}:=\beta^{-\frac{11}{10}}\left(\partial_{y}^{2} \partial_{\theta} v\right)\left(v^{-1} \partial_{y} F_{2}\right)\left(v^{-1} \partial_{y} \partial_{\theta}^{3} v\right)
$$


which appear in $\beta^{-\frac{11}{10}} E_{2,1,0}\left(\partial_{y}^{2} \partial_{\theta} v\right)$.

For $W_{1}$, a suitable estimate for $\partial_{\theta} F_{1}$, namely

$$
\left|\partial_{\theta} F_{1}\right| \lesssim\left|\partial_{p} F_{1}\right|\left|\partial_{y} \partial_{\theta} v\right|+\left|\partial_{q} F_{1}\right|\left|\partial_{\theta} q\right| \lesssim \frac{\left(\beta_{0}+\varepsilon_{0}\right)^{\frac{1}{20}}}{1+p^{2}+q^{2}}
$$

follows from the smallness component of Condition [C3]. Applying Cauchy-Schwarz then yields

$$
\begin{aligned}
\left|W_{1}\right| & \leq\left(\beta_{0}+\varepsilon_{0}\right)^{\frac{1}{20}} \beta^{-\frac{11}{10}} \frac{\left(\partial_{y}^{2} \partial_{\theta} v\right)^{2}+\left(\partial_{y}^{4} v\right)^{2}}{1+p^{2}+q^{2}} \\
& \leq\left(\beta_{0}+\varepsilon_{0}\right)^{\frac{1}{20}}\left[\Upsilon_{3}+\beta^{-\frac{11}{10}} B_{3,0,0}\right]
\end{aligned}
$$

where $\beta^{-\frac{11}{10}} B_{3,0,0}$ appears in the evolution equation for $\beta^{-\frac{11}{10}}\left(\partial_{y}^{3} v\right)^{2}$.

For $W_{2}$, we get good decay from the term $v^{-1} \partial_{y} F_{2}$. By Theorem 4.6, one has

$$
\left|v^{-1} \partial_{y} F_{2}\right| \leq \frac{\beta^{\frac{3}{5}}}{1+p^{2}+q^{2}}
$$

and hence

$$
\left|W_{2}\right| \leq \beta^{-\frac{1}{2}} \frac{\left|\partial_{y}^{2} \partial_{\theta} v\right|\left|v^{-1} \partial_{y} \partial_{\theta}^{3} v\right|}{1+p^{2}+q^{2}} \leq \beta^{\frac{1}{20}}\left[\Upsilon_{3}+B_{1,2,0}\right],
$$

where $B_{1,2,0}$ appears in inequality (7.5).

The remaining terms in inequality (7.6) are estimated by the same methods we employed many times in previous sections. Combining these estimates with inequalities (7.7)-(7.8) yields

$$
\partial_{\tau} \Upsilon_{3} \leq A_{v} \Upsilon_{3}-\frac{1}{2} \beta^{-\frac{11}{10}} B_{2,1,0}+\beta^{\frac{1}{20}} B_{1,2,0}+\left(\beta_{0}+\varepsilon_{0}\right)^{\frac{1}{20}} \beta^{-\frac{11}{10}} B_{3,0,0}+\beta^{\frac{11}{10}},
$$

where $-\frac{1}{2} \Upsilon_{3}$ was used to control various terms in $W_{1}$ and $W_{2}$.

By similar techniques, we estimate that $\Upsilon_{1}:=\left(v^{-1} \partial_{\theta}^{3} v\right)^{2}$ and $\Upsilon_{4}:=\beta^{-\frac{11}{10}}\left(\partial_{y}^{3} v\right)^{2}$ satisfy the differential inequalities

$$
\partial_{\tau} \Upsilon_{1} \leq A_{v} \Upsilon_{1}-\frac{1}{2} B_{0,3,1}+\left(\beta_{0}+\varepsilon_{0}\right)^{\frac{1}{20}} B_{1,2,0}+\beta^{\frac{11}{10}}
$$

and

$$
\partial_{\tau} \Upsilon_{4} \leq A_{v} \Upsilon_{4}-\frac{1}{2} \beta^{-\frac{11}{10}} B_{3,0,0}+\left(\beta_{0}+\varepsilon_{0}\right)^{\frac{1}{20}} \beta^{-\frac{11}{10}} B_{2,1,0}+\beta^{\frac{11}{10}},
$$

respectively. Adding equations (7.10), (7.5), (7.9), and (7.11) completes the proof.

\section{The SECOND Bootstrap MACHINE}

In this section and those that follow, we describe the asymptotic behavior of solutions. Specifically, we show that a solution $v$ to equation (2.5), which is a rescaling of a solution $u$ to equation (2.1), may be decomposed into a slowly-changing main component and a rapidly-decaying small component. We accomplish this by building a second bootstrap machine, following [9]. 
8.1. Input. Our first input to this bootstrap machine is that $u(x, t)$ is a solution of equation (2.1) satisfying the following conditions:

[Cd] There exists $t_{\#}>0$ such that for $0 \leq t \leq t_{\#}$, there exist $C^{1}$ functions $a(t)$ and $b(t)$ such that $u(x, \theta, t)$ admits the decomposition

$$
u(x, \theta, t)=\lambda(t) v(y, \theta, \tau)=\lambda(t)\left[\left(\frac{2+b(t) y^{2}}{a(t)+\frac{1}{2}}\right)^{\frac{1}{2}}+\phi(y, \theta, \tau)\right]
$$

with the $L^{2}$ orthogonality properties

$$
\phi(\cdot, \cdot, \tau) \perp e^{-\frac{a(t)}{2} y^{2}},\left(1-a(t) y^{2}\right) e^{-\frac{a(t)}{2} y^{2}},
$$

where $a(t):=-\lambda(t) \partial_{t} \lambda(t), y:=\lambda^{-1}(t) x$, and $\tau(t):=\int_{0}^{t} \lambda^{-2}(s) \mathrm{d} s$.

Proposition 8.3 will show that Condition [Cd] follows from our Main Assumptions.

To state the second set of inputs, we define estimating functions to control the quantities $\phi(y, \theta, \tau), a(t(\tau))$, and $b(t(\tau))$ appearing in equation (8.1), namely:

$$
\begin{aligned}
M_{m, n}(T) & :=\max _{\tau \leq T} \beta^{-\frac{m+n}{2}-\frac{1}{10}}(\tau)\|\phi(\cdot, \cdot, \tau)\|_{m, n}, \\
A(T) & :=\max _{\tau \leq T} \beta^{-2}(\tau)\left|a(t(\tau))-\frac{1}{2}+b(t(\tau))\right|, \\
B(T) & :=\max _{\tau \leq T} \beta^{-\frac{3}{2}}(\tau)|b(t(\tau))-\beta(\tau)| .
\end{aligned}
$$

where $(m, n) \in\{(3,0),(11 / 10,0),(2,1),(1,1)\}$. Here we used the definitions

$$
\|\phi\|_{m, n}:=\left\|\langle y\rangle^{-m} \partial_{y}^{n} \phi\right\|_{L^{\infty}} \quad \text { and } \quad \beta(\tau):=\frac{1}{\frac{1}{b(0)}+\tau} .
$$

By standard regularity theory for quasilinear parabolic equations, if the initial data satisfy the Main Assumptions in Section 2 for $b_{0}$ and $c_{0}$ sufficiently small, then (making $t_{\#}>0$ smaller if necessary) the solution will satisfy the second set of inputs for this bootstrap argument, namely:

[Cb] For any $\tau \leq \tau\left(t_{\#}\right)$, one has

$$
A(\tau)+B(\tau)+|M(\tau)| \lesssim \beta^{-\frac{1}{20}}(\tau)
$$

where $M$ denotes the vector

$$
M:=\left(M_{i, j}\right),(i, j) \in\{(3,0),(11 / 10,0),(2,1),(1,1)\} .
$$

Remark 8.1. Condition [Cb] implies estimates on $v$ and $v^{-\frac{1}{2}} \partial_{y} v$ in the inner region $\beta y^{2} \leq 20$, which in turn imply Condition [C0i], the estimate $\left|\partial_{y} v\right| \lesssim \beta^{\frac{1}{2}} v^{\frac{1}{2}}$ of Condition [C1i], and the estimate $\langle y\rangle^{-1}\left|\partial_{y} v\right| \lesssim \beta$ of Condition [Cg].

The final inputs to this bootstrap machine are the following estimates. They follow from the outputs of the first bootstrap machine, as summarized in Section 4.2. For any $\tau \in\left[0, \tau\left(t_{\#}\right)\right]$, the estimates proved in Sections $4-7$ show that $v$ satisfies

$$
v(y, \theta, \tau) \geq 1
$$


and that there exist constants $\epsilon_{0} \ll 1$ and $C$, independent of $\tau\left(t_{\#}\right)$, such that

$$
\begin{gathered}
\left|\partial_{y} v\right| \leq C \\
\left|v^{-1} \partial_{\theta}^{2} v\right|,\left|\partial_{y} \partial_{\theta}^{2} v\right| \leq \epsilon_{0} \ll 1 \\
v^{-1}\left|\partial_{y} v\right| \leq C \beta^{\frac{1}{2}},\left|\partial_{y}^{2} v\right| \leq C \beta^{\frac{13}{20}},\left|\partial_{y}^{3} v\right| \leq C \beta^{\frac{23}{20}} \\
v^{-2}\left|\partial_{y} \partial_{\theta}^{2} v\right|, v^{-1}\left|\partial_{y} \partial_{\theta} v\right|, v^{-1}\left|\partial_{y}^{2} \partial_{\theta} v\right|, v^{-2}\left|\partial_{\theta}^{2} v\right| \leq C \beta^{\frac{33}{20}}
\end{gathered}
$$

8.2. Output. The main result of this section is:

Theorem 8.2. Suppose that Conditions $[C d]$ and $[C b]$ and estimates (8.7)-(8.11) hold in an interval $\tau \in\left[0, \tau_{1}\right]$. Then there exists $C$ independent of $\tau_{1}$ such that for the same time interval, the parameters $a$ and $b$ and the function $\phi$ are such that

$$
A(\tau)+B(\tau)+|M(\tau)| \leq C .
$$

This theorem will be reformulated into Propositions 9.1 and 9.2, and then proved in Section 9.1.

8.3. Structure. Before giving the details of the second bootstrap argument, we discuss the general strategy of its proof. The first observation is that our Main Assumptions imply that there exist $\lambda(t)$ and $b(t)$ such that the solution $v(\cdot, \cdot, \tau)$ remains close to the adiabatic approximation $V_{a(t(\tau)), b(t(\tau))}$ defined in equation (2.9) for at least a short time. Moreover, we can choose those parameters so that the solution admits the decomposition in equation (8.1), subject to the orthogonality stipulations of Condition [Cd].

It is shown in [9] that $\lambda(t)$ and $b(t)$ can be chosen so that Condition [Cd] is satisfied. To state this precisely, we need some definitions. Given any time $t_{0}$ and $\delta>0$, we define $I_{t_{0}, \delta}:=\left[t_{0}, t_{0}+\delta\right]$. We say that $\lambda(t)$ is admissible on $I_{t_{0}, \delta}$ if $\lambda \in C^{2}\left(I_{t_{0}, \delta}, \mathbb{R}_{+}\right)$and $a(t):=-\lambda \partial_{t} \lambda \in[1 / 4,1]$. Proposition 5.3 and Lemma 5.4 of [9] imply the following result.

Proposition 8.3. Given $t_{*}>0$, fix $t_{0} \in\left[0, t_{*}\right)$ and $\lambda_{0}>0$. Then there exist $\delta, \varepsilon>0$ and a function $\lambda(t)$, admissible on $I_{t_{0}, \delta}$, such that if

(I) $\langle x\rangle^{-3} u \in C^{1}\left(\left[0, t_{*}\right), L^{\infty}\left(\mathbb{S}^{1} \times \mathbb{R}\right)\right)$,

(II) $\inf u>0$, and

(III) $\left\|v\left(\cdot, \cdot, t_{0}\right)-V_{a_{0}, b_{0}}\right\|_{3,0} \ll b_{0}$ for some $a_{0} \in[1 / 4,1]$ and $b_{0} \in(0, \varepsilon]$, where $v(y, \theta, \tau)=\lambda(t)^{-1} u(\lambda(t) x, \theta, t)$, then there exist

$$
a(\tau(t)) \in C^{1}\left(I_{t_{0}, \delta},[1 / 4,1]\right) \quad \text { and } \quad b(\tau(t)) \in C^{1}\left(I_{t_{0}, \delta},(0, \varepsilon]\right)
$$

such that the rescaled solution $v(y, \theta, \tau)$ admits the decomposition and orthogonality relations of Condition [Cd], with $\lambda(0)=\lambda_{0}$ and $a(\tau(t))=-\lambda(t) \partial_{t} \lambda(t)$.

The second bootstrap machine will establish that $V_{a(t(\tau)), b(t(\tau))}$ is the large, slowly-changing part of the solution, while $\phi$ is the small, rapidly-decaying part. The utility of the stipulated orthogonality will become clear in Section 11, when we compute the linearization of an equation closely related to (2.5).

In what follows, we will usually convert from the time scale $t$ to $\tau$. To study the asymptotics of the solution, we derive equations for $a_{\tau}, b_{\tau}$, and $\phi$ in equations (9.3), (9.8) and (9.9), respectively. Then we analyze those equations to show that

$$
a(\tau) \rightarrow \frac{1}{2}, \quad b(\tau) \approx \frac{1}{\frac{1}{b_{0}}+\tau}=: \beta(\tau), \quad \text { and } \quad\left\|\langle y\rangle^{-m} \partial_{y}^{n} \phi\right\|_{\infty} \lesssim \beta^{\frac{m+n}{2}+\frac{1}{10}}
$$


for $(m, n)=(3,0),(11 / 10,0),(2,1)$ and $(1,2)$.

The first two estimates above are proved in Section 10. Their proofs are straightforward generalizations of those in [9]. The third estimate, which establishes the fast decay of $\phi$, is the most critical. It is proved in Section 11. For all three results, the crucial new ingredients from [9] are the $\theta$-derivative bounds appearing in estimates (8.8)-(8.11), which follow from the first bootstrap machine constructed in Sections $4-7$.

\section{EVOlution EQUATIONS FOR THE DECOMPOSITION}

Equation (2.5) is not self-adjoint with respect to its linearization, but it can be made so by a suitable gauge transformation. By Condition [Cd] in Section 8 , there exists a ( $t$-scale) time $0<t_{\#} \leq \infty$ such that the gauge-fixed quantity

$$
w(y, \theta, \tau):=v(y, \theta, \tau) e^{-\frac{a}{4} y^{2}}
$$

can be decomposed as

$$
w=w_{a b}(y)+\xi(y, \theta, \tau), \quad \text { with } \quad \xi \perp \phi_{0, a}, \phi_{2 a} .
$$

Here

$$
\phi_{0, a}:=\left(\frac{a}{2 \pi}\right)^{\frac{1}{4}} e^{-\frac{a y^{2}}{4}} \quad \text { and } \quad \phi_{2, a}:=\left(\frac{a}{8 \pi}\right)^{\frac{1}{4}}\left(1-a y^{2}\right) e^{-\frac{a y^{2}}{4}},
$$

and the orthogonality is with respect to the $L^{2}\left(\mathbb{S}^{1} \times \mathbb{R}\right)$ inner product. The parameters $a$ and $b$ are $C^{1}$ functions of $t$; the (almost stationary) part is

$$
w_{a b}:=\sqrt{2+b y^{2} / a+\frac{1}{2}} e^{-\frac{a}{4} y^{2}} ;
$$

and the (rapidly-decaying) fluctuation is

$$
\xi:=e^{-\frac{a y^{2}}{4}} \phi
$$

To simplify notation, we will write $a(\tau)$ and $b(\tau)$ for $a(t(\tau))$ and $b(t(\tau))$, respectively. (This will not cause confusion, as the original functions $a(t)$ and $b(t)$ are not needed until Section 12.) In this section, we derive evolution equations for the parameters $a(\tau)$ and $b(\tau)$, and the fluctuation $\xi(y, \theta, \tau)$.

We substitute equation (9.1) into equation (2.5) to obtain the following equation for $\xi$,

$$
\partial_{\tau} \xi=-L(a, b) \xi+F(a, b)+N_{1}(a, b, \xi)+N_{2}(a, b, \xi)+N_{3}(a, b, \xi),
$$

where $L(a, b)$ is the linear operator given by

$$
L(a, b):=-\partial_{y}^{2}+\frac{a^{2}+\partial_{\tau} a}{4} y^{2}-\frac{3 a}{2}-\frac{\frac{1}{2}+a}{2+b y^{2}}-\frac{1}{2} \partial_{\theta}^{2},
$$

and the functions $F(a, b)$ and $N_{i}(a, b, \xi),(i=1,2,3)$, are given below. We define

$$
F(a, b):=\frac{1}{2} e^{-\frac{a y^{2}}{4}}\left(\frac{2+b y^{2}}{a+\frac{1}{2}}\right)^{\frac{1}{2}}\left[\Gamma_{1}+\Gamma_{2} \frac{y^{2}}{2+b y^{2}}-\frac{b^{3} y^{4}}{\left(2+b y^{2}\right)^{2}}\right],
$$


with

$$
\begin{aligned}
\Gamma_{1} & :=\frac{\partial_{\tau} a}{a+\frac{1}{2}}+a-\frac{1}{2}+b, \\
\Gamma_{2} & :=-\partial_{\tau} b-b\left(a-\frac{1}{2}+b\right)-b^{2}, \\
N_{1}(a, b, \xi) & :=-\frac{1}{v} \frac{a+\frac{1}{2}}{2+b y^{2}} e^{\frac{a y^{2}}{4}} \xi^{2}, \\
N_{2}(a, b, \xi) & :=-e^{-\frac{a y^{2}}{4}} \frac{p^{2}}{1+p^{2}+q^{2}} \partial_{y}^{2} v .
\end{aligned}
$$

The final term, $N_{3}$, did not appear in [9]; it is

$$
\begin{aligned}
N_{3}(a, b, \xi):=\quad\left[v^{-2} \frac{1+p^{2}}{1+q^{2}+p^{2}}-\frac{1}{2}\right] \partial_{\theta}^{2} v e^{-\frac{a y^{2}}{4}} \\
+e^{-\frac{a y^{2}}{4}} v^{-1} \frac{2 p q}{1+p^{2}+q^{2}} \partial_{\theta} \partial_{y} v \\
+e^{-\frac{a y^{2}}{4}} v^{-2} \frac{q}{1+p^{2}+q^{2}} \partial_{\theta} v
\end{aligned}
$$

Now we derive differential equations for the parameters $a$ and $b$. Taking inner products on equation (9.3) with the functions $\phi_{k, a},(k=0,2)$, and using the orthogonality conditions $\xi \perp \phi_{0, a}, \phi_{2, a}$ in (9.1), one obtains two equations:

$$
\begin{aligned}
\left\langle F(a, b), \phi_{0, a}\right\rangle= & -\left\langle\xi, \partial_{\tau} \phi_{0, a}\right\rangle-\left\langle\left(\frac{\frac{1}{2}+a}{2+b y^{2}}-\frac{\frac{1}{2}+a}{2}\right) \xi, \phi_{0, a}\right\rangle \\
& -\sum_{k=1}^{3}\left\langle N_{k}, \phi_{0, a}\right\rangle+\frac{a_{\tau}}{4}\left\langle\xi, y^{2} \phi_{0, a}\right\rangle, \\
\left\langle F(a, b), \phi_{2, a}\right\rangle= & -\left\langle\xi, \partial_{\tau} \phi_{2, a}\right\rangle-\left\langle\left(\frac{\frac{1}{2}+a}{2+b y^{2}}-\frac{\frac{1}{2}+a}{2}\right) \xi, \phi_{2, a}\right\rangle \\
& -\sum_{k=1}^{3}\left\langle N_{k}, \phi_{2, a}\right\rangle+\frac{a_{\tau}}{4}\left\langle\xi, y^{2} \phi_{2, a}\right\rangle .
\end{aligned}
$$

Note that the terms on the right-hand sides of equations (9.8)-(9.9) depend on $\xi$, while $F(a, b)$ depends on $a_{\tau}, b_{\tau}, a, b$ and $y$.

We now show that Theorem 8.2 is a consequence of the following two results.

Proposition 9.1. Suppose Conditions [Cd] and [Cb] and estimates (8.8)-(8.11) hold. Then there exists a nondecreasing polynomial $P(M, A)$ of $A$ and the components of $M$ such that

$$
B(\tau) \lesssim 1+P(M(\tau), A(\tau))
$$

and

$$
A(\tau) \lesssim A(0)+\beta^{\frac{7}{10}}(0) P(M(\tau), A(\tau)) .
$$

This will be proved in Section 10. 
Proposition 9.2. Suppose Conditions [Cd] and [Cb] and estimates (8.8)-(8.11) hold. Then the function $\phi$ satisfies the estimates

$$
\begin{gathered}
M_{3,0}(\tau) \lesssim M_{3,0}(0)+\beta^{\frac{1}{20}}(0) P(M(\tau), A(\tau)), \\
M_{\frac{11}{10}, 0}(\tau) \lesssim M_{\frac{11}{10}, 0}(0)+1+\epsilon_{0} M_{\frac{11}{10}, 0}+M_{3,0}(\tau)+\beta^{\frac{1}{20}}(0) P(M(\tau), A(\tau)), \\
M_{2,1}(\tau) \lesssim M_{2,1}(0)+M_{3,0}(\tau)+\beta^{\frac{1}{20}}(0) P(M(\tau), A(\tau))
\end{gathered}
$$

and

$$
M_{1,1}(\tau) \lesssim M_{1,2}(0)+M_{3,0}(\tau)+M_{2,1}(\tau)+\beta^{\frac{1}{20}}(0) P(M(\tau), A(\tau)),
$$

for any $\tau \in\left[0, \tau\left(t_{\#}\right)\right]$, where $P(M, A)$ is a nondecreasing polynomial of $A$ and the components of $M$.

This will be proved in Section 11.

These two propositions imply Theorem 8.2, as we now show.

Proof of Theorem 8.2. Observe that $M_{3,0}(\tau)$ and $M_{2,1}(\tau)$ are present on the righthand sides of equations (9.13)-(9.15). To remove these, we use estimates (9.12) and (9.14) to recast estimates (9.10), (9.11), and (9.12)-(9.15) as

$$
\begin{gathered}
A(\tau)+|M(\tau)| \lesssim A(0)+|M(0)|+\beta^{\frac{1}{10}}(0) P(|M(\tau)|, A(\tau)), \\
B(\tau) \lesssim 1+P(|M(\tau)|, A(\tau)),
\end{gathered}
$$

with $P$ being some polynomial. By the boundedness of $|M(0)|, A(0)$ and the smallness of $\beta_{0}$, we obtain the desired estimate (8.12).

\section{Estimates to CONTROL THE PARAMETERS $a$ AND $b$}

We start by stating a preliminary estimate, which is proved later in this section.

Lemma 10.1. The functions $\Gamma_{1}$ and $\Gamma_{2}$ appearing in definition (9.4) satisfy

$$
\left|\Gamma_{1}\right|,\left|\Gamma_{2}\right| \lesssim \beta^{\frac{27}{10}} P(M, A) .
$$

Now we use estimate (10.1) to prove Proposition 9.1.

Proof of Proposition 9.1. We start by proving estimate (9.10).

Recall that $\Gamma_{1}$ and $\Gamma_{2}$ are defined in terms of $a_{\tau}, b_{\tau}, a$ and $b$. Thus we begin by rewriting $\Gamma_{2}$ in estimate (10.1) as

$$
\left|\partial_{\tau} b+b^{2}\right| \lesssim b\left|\frac{1}{2}-a+b\right|+\beta^{\frac{27}{10}} P(M, A) .
$$

The first term on the RHS is bounded by $b \beta^{2} A \lesssim \beta^{3} A$ by definition of $A$. Hence

$$
\left|\partial_{\tau} b+b^{2}\right| \lesssim \beta^{\frac{27}{10}} P(M, A)
$$

Divide estimate (10.2) by $b^{2}$, and use the inequality $\beta \lesssim b$ implied by the condition $B \leq \beta^{-\frac{1}{20}}$, to obtain

$$
\left|\partial_{\tau} \frac{1}{b}-1\right| \lesssim \beta^{\frac{7}{10}} P(M, A)
$$


Then use the fact that $\beta$ satisfies $-\partial_{\tau} \beta^{-1}+1=0$ to get

$$
\left|\partial_{\tau}\left(\frac{1}{b}-\frac{1}{\beta}\right)\right| \lesssim \beta^{\frac{7}{10}} P(M, A) .
$$

Integrating this estimate over $[0, \tau]$ and using the facts that $b \lesssim \beta$ and $\beta(0)=b(0)$, we obtain

$$
\beta^{-\frac{3}{2}}|\beta-b| \lesssim \beta^{\frac{1}{2}} \int_{0}^{\tau} \beta^{\frac{7}{10}}(s) P(M(s), A(s)) \mathrm{d} s \lesssim \beta^{\frac{1}{2}} P(M(\tau), A(\tau)),
$$

which together with the definitions of $\beta$ and $B$ implies estimate (9.10).

Now we turn to estimate (9.11). To facilitate later discussions, we define

$$
\Gamma:=a-\frac{1}{2}+b
$$

Differentiating $\Gamma$ with respect to $\tau$, writing $\partial_{\tau} b$ and $\partial_{\tau} a$ in terms of $\Gamma_{1}$ and $\Gamma_{2}$, and using estimate (10.1), we obtain

$$
\partial_{\tau} \Gamma+\left(a+\frac{1}{2}+b\right) \Gamma=-b^{2}+\mathcal{R}_{b},
$$

where $\mathcal{R}_{b}$ obeys the bound

$$
\left|\mathcal{R}_{b}\right| \leq \beta^{\frac{27}{10}} P(M, A) .
$$

Let $\mu=e^{\int_{0}^{\tau} a(s)+\frac{1}{2}+b(s) \mathrm{d} s}$. Then the equation above implies that

$$
\mu \Gamma=\Gamma_{0}-\int_{0}^{\tau} \mu b^{2} \mathrm{~d} s+\int_{0}^{\tau} \mu \mathcal{R}_{b} \mathrm{~d} s .
$$

We now use the inequality $b \lesssim \beta$ and the bound for $\mathcal{R}_{b}$ to estimate over $[0, \tau] \leq[0, T]$ that

$$
|\Gamma| \lesssim \mu^{-1} \Gamma_{0}+\mu^{-1} \int_{0}^{\tau} \mu \beta^{2} \mathrm{~d} s+\mu^{-1}\left[\int_{0}^{\tau} \mu \beta^{\frac{27}{10}} \mathrm{~d} s\right] P(M, A) .
$$

For our purposes, it is sufficient to use the weaker inequality

$$
|\Gamma| \lesssim \mu^{-1} \Gamma(0)+\mu^{-1} \int_{0}^{\tau} \mu \beta^{2} \mathrm{~d} s\left[1+\beta^{\frac{7}{10}}(0) P(M, A)\right] .
$$

The conditions $A(\tau), B(\tau) \leq \beta^{-\frac{1}{20}}(\tau)$ imply that $a+\frac{1}{2}+b \geq \frac{1}{2}$. Thus, it is not difficult to show that $\beta^{-2} \mu^{-1} \Gamma(0) \leq A(0)$ and $\beta^{-2} \mu^{-1} \int_{0}^{\tau} \mu \beta^{2} \mathrm{~d} s$ are bounded. Hence we have

$$
A \lesssim A(0)+1+\beta^{\frac{7}{10}}(0) P(M, A),
$$

which is estimate (9.11).

This completes the proof of Proposition 9.1.

Now we present:

Proof of Lemma 10.1. We begin by analyzing equations (9.8)-(9.9). For notational purpose, we denote the terms on their right-hand sides by $G_{1}$ and $G_{2}$, namely

$$
\begin{aligned}
\left\langle F(a, b), \phi_{0, a}\right\rangle & =G_{1}, \\
\left\langle F(a, b), \phi_{2, a}\right\rangle & =G_{2} .
\end{aligned}
$$


We start by bounding the terms in $G_{1}$ and $G_{2}$. Using estimates (8.11) and (4.7), both of which follow from the first bootstrap machine, one finds for $k=0,2$ that

$$
\begin{aligned}
\left|\left\langle v^{-1} F_{3} \partial_{y} \partial_{\theta} v, \phi_{k, a}\right\rangle\right|+ & \left|\left\langle v^{-2} F_{4} \partial_{\theta} v, \phi_{k, a}\right\rangle\right| \\
& \lesssim\left|v^{-2} \partial_{\theta} v\right|\left(\left|v^{-2} \partial_{y} \partial_{\theta} v\right|+\left|v^{-2} \partial_{\theta} v\right|\right) \lesssim \beta^{\frac{33}{10}} .
\end{aligned}
$$

For the term $\left\langle v^{-2} F_{2} \partial_{\theta}^{2} v, \phi_{k, a}\right\rangle$, we integrate by parts in $\theta$ to generate sufficient decay estimates, obtaining

$$
\begin{aligned}
& \left|\left\langle v^{-2} \frac{1+p^{2}}{1+p^{2}+q^{2}} \partial_{\theta}^{2} v, e^{-\frac{a}{4} y^{2}}\right\rangle\right| \\
& =\left|\left\langle\partial_{\theta} v, \partial_{\theta}\left[v^{-2} \frac{1+p^{2}}{1+p^{2}+q^{2}} e^{-\frac{a}{4} y^{2}}\right]\right\rangle\right| \\
& =\left|\left\langle 2 v^{-3} \frac{1+p^{2}}{1+p^{2}+q^{2}}\left(\partial_{\theta} v\right)^{2}+v^{-2} \frac{2 p q \partial_{y} \partial_{\theta} v-2\left(1+p^{2}\right)\left(v^{-1} \partial_{\theta}^{2} v-2 q^{2}\right)}{\left(1+p^{2}+q^{2}\right)^{2}} \partial_{\theta} v, e^{-\frac{a}{4} y^{2}}\right\rangle\right| \\
& \lesssim \beta^{\frac{33}{10}} .
\end{aligned}
$$

In the last step, we applied estimate (8.11). By similar methods, i.e. integration by parts, we get

$$
\left|\left\langle v^{-2} \frac{1+p^{2}}{1+p^{2}+q^{2}} \partial_{\theta}^{2} v,\left(a y^{2}-1\right) e^{-\frac{a}{4} y^{2}}\right\rangle\right| \lesssim \beta^{\frac{33}{10}} .
$$

For the terms $N_{1}$ and $N_{2}$, we have

$$
\left|\left\langle N_{1}, \phi_{k, a}\right\rangle\right| \lesssim\left\|\langle y\rangle^{-6} N_{1}\right\|_{\infty} \leq M_{3,0}^{2} \beta^{\frac{16}{5}}, \quad(k=0,2) .
$$

Then we use the estimate $\left|\partial_{y}^{2} v\right| \lesssim \beta^{\frac{13}{20}}$ of (8.10) to obtain

$$
\begin{aligned}
\left|\left\langle N_{2}, \phi_{k, a}\right\rangle\right|\left\|\langle y\rangle^{-2} p^{2} \partial_{y}^{2} v\right\|_{\infty} & \leq\left\|\langle y\rangle^{-3} \partial_{y} v\right\|_{\infty}^{2}\left\|\partial_{y}^{2} v\right\|_{\infty} \\
& \lesssim\left(\beta+M_{2,1} \beta^{\frac{16}{10}}\right)^{2} \beta^{\frac{13}{20}}, \quad(k=0,2) .
\end{aligned}
$$

In estimating $\langle y\rangle^{-3} \partial_{y} v$, we used $v=\sqrt{2+b y^{2} / a+\frac{1}{2}}+e^{\frac{a}{4} y^{2}}$ and the facts $a \approx \frac{1}{2}$, $b \approx \beta$ implied by the consequences $A, B \lesssim \beta^{-\frac{1}{20}}$ of estimate (8.5) and the definition of $M_{3,0}$ in (8.2).

We collect the estimates above, using our conditions on $A(\tau)$ and $B(\tau)$, to get

$$
G_{1}, G_{2} \lesssim\left|\partial_{\tau} a\right| \beta^{2} M_{3,0}+\beta^{\frac{53}{20}}\left(1+M_{2,1}^{2}+M_{3,0}^{2}\right) .
$$

Now we turn to the terms on the left-hand sides of equations (10.5)-(10.6). We decompose the function $F(a, b)$ defined in equation (9.4) into components approximately parallel or orthogonal to $\phi_{0, a}$ and $\phi_{2, a}$, plus a term of order $b^{3}$, as follows:

$$
F(a, b)=\left(\frac{2+b y^{2}}{a+\frac{1}{2}}\right)^{\frac{1}{2}} \frac{1}{2} e^{-\frac{a y^{2}}{4}}\left[\Gamma_{1}+\Gamma_{2} \frac{1}{a\left[2+b y^{2}\right]}+\Gamma_{2} \frac{a y^{2}-1}{a\left[2+b y^{2}\right]}-\frac{b^{3} y^{4}}{\left(2+b y^{2}\right)^{2}}\right] .
$$

Consequently, one has

$$
\left|\left\langle F(a, b), \phi_{0, a}\right\rangle\right| \gtrsim\left|\Gamma_{1}+\frac{1}{2 a} \Gamma_{2}\right|-|b|\left(\left|\Gamma_{1}\right|+\left|\Gamma_{2}\right|\right)-b^{3}
$$


and

$$
\left|\left\langle F(a, b), \phi_{2, a}\right\rangle\right| \gtrsim\left|\Gamma_{2}\right|-|b|\left(\left|\Gamma_{1}\right|+\left|\Gamma_{2}\right|\right)-b^{3}
$$

which together with estimate (10.7) imply that

$$
\left|\Gamma_{1}\right|,\left|\Gamma_{2}\right| \lesssim \beta^{\frac{53}{20}}\left(1+M_{2,1}^{2}+M_{3,0}^{2}\right)+\left|\partial_{\tau} a\right| \beta^{2} M_{3,0}
$$

To control the term $\left|\partial_{\tau} a\right|$ on the RHS, we use the definitions of $\Gamma_{1}$ and $\partial_{\tau} a$ to obtain

$$
\left|\partial_{\tau} a\right| \lesssim\left|\Gamma_{1}\right|+\beta^{2} A
$$

Hence we have

$$
\left|\Gamma_{1}\right|,\left|\Gamma_{2}\right| \lesssim \beta^{\frac{53}{20}}\left(1+M_{2,1}^{2}+M_{3,0}^{2}\right)+\beta^{4} A M_{3,0}
$$

This together with the condition $|M(\tau)| \leq \beta^{-\frac{1}{10}}(\tau)$ implies estimate (10.1).

To facilitate later discussions, we state some other useful estimates for $F$.

Corollary 10.2. If $(m, n) \in\{(3,0),(11 / 10,0),(2,1),(1,1)\}$ and $A(\tau), B(\tau) \lesssim$ $\beta^{-\frac{1}{20}}(\tau)$, then

$$
\left\|e^{\frac{a y^{2}}{4}} F(a, b)\right\|_{m, n} \lesssim \beta^{\frac{m+n+1}{2}}(\tau) P(M, A) .
$$

Proof. In what follows, we only prove the case $(m, n)=(11 / 10,0)$. The proofs of the remaining cases are similar (see [9]), hence omitted.

Recalling definition (9.4) for $F$, one observes that

$$
\left\|\langle y\rangle^{-\frac{11}{10}} \frac{y^{2}}{\left(2+b y^{2}\right)^{\frac{1}{2}}}\right\|_{\infty} \leq b^{-\frac{9}{20}} \lesssim \beta^{-\frac{9}{20}}
$$

Thus the estimates for $\Gamma_{1}$ and $\Gamma_{2}$ in (10.1) imply that

$$
\left\|\langle y\rangle^{-\frac{11}{10}}\left(\frac{2+b y^{2}}{a+\frac{1}{2}}\right)^{\frac{1}{2}}\left[\Gamma_{1}+\Gamma_{2} \frac{y^{2}}{2+b y^{2}}\right]\right\|_{\infty} \lesssim\left(\left|\Gamma_{1}\right|+\left|\Gamma_{2}\right|\right) \beta^{-\frac{9}{20}} \lesssim \beta^{\frac{11}{5}} P(M, A) .
$$

By similar reasoning, one has

$$
\left\|\langle y\rangle^{-\frac{11}{10}} \frac{b^{3} y^{4}}{a\left(2+b y^{2}\right)^{2}}\left(\frac{2+b y^{2}}{a+\frac{1}{2}}\right)^{\frac{1}{2}}\right\|_{\infty} \lesssim \beta^{\frac{21}{20}} .
$$

Combining the estimates above, we complete the estimate for $(m, n)=(11 / 10,0)$.

\section{Estimates to Control the fluctuation $\phi$}

In this section, we prove the estimates that make up Proposition 9.2.

Recall the evolution equation (9.3) satisfied by $\xi(y, \theta, \tau)$. We begin our analysis by reparameterizing $\xi$ so that critical terms in the resulting (new) evolution equation have time-independent coefficients.

Recall that $\tau(t):=\int_{0}^{t} \lambda^{-2}(s) \mathrm{d} s$ for any $\tau \geq 0$, and $a(\tau):=-\lambda(t(\tau)) \partial_{t} \lambda(t(\tau))$. Let $t(\tau)$ be the inverse function to $\tau(t)$, and pick $T>0$. We approximate $\lambda(t(\tau))$ on the interval $0 \leq \tau \leq T$ by a new trajectory $\lambda_{1}(t(\tau))$, chosen so that $\lambda_{1}(t(T))=$ $\lambda(t(T))$ and $\alpha:=-\lambda_{1}(t(\tau)) \partial_{t} \lambda_{1}(t(\tau))=a(T)$ is constant.

Then we introduce new independent variables $z(x, t):=\lambda_{1}^{-1}(t) x$ and $\sigma(t):=$ $\int_{0}^{t} \lambda_{1}^{-2}(s) \mathrm{d} s$, together with a new function $\eta(z, \theta, \sigma)$ defined by

$$
\lambda_{1}(t) e^{\frac{\alpha}{4} z^{2}} \eta(z, \theta, \sigma):=\lambda(t) e^{\frac{a(\tau)}{4} y^{2}} \xi(y, \theta, \tau) \equiv \lambda(t) \phi(y, \theta, \tau) .
$$


One should keep in mind that the variables $z$ and $y, \sigma, \tau$ and $t$ are related by $z=\frac{\lambda(t)}{\lambda_{1}(t)} y, \sigma(t):=\int_{0}^{t} \lambda_{1}^{-2}(s) \mathrm{d} s$, and $\tau=\int_{0}^{t} \lambda^{-2}(s) \mathrm{d} s$.

For any $\tau=\int_{0}^{t(\tau)} \lambda^{-2}(s) \mathrm{d} s$ with $t(\tau) \leq t(T)$, or equivalently $\tau \leq T$, we define a new function $\sigma(\tau):=\int_{0}^{t(\tau)} \lambda_{1}^{-2}(s) \mathrm{d} s$. Observing that the function $\sigma$ is invertible, we denote its inverse by $\tau(\sigma)$.

The new function $\eta$ satisfies the equation

$$
\begin{aligned}
\partial_{\sigma} \eta= & -\mathcal{L}_{\alpha} \eta(\sigma)+\mathcal{W}(a, b) \eta(\sigma)+\mathcal{F}(a, b)(\sigma) \\
& +\mathcal{N}_{1}(a, b, \eta)+\mathcal{N}_{2}(a, b, \eta)+\mathcal{N}_{3}(a, b, \eta),
\end{aligned}
$$

where the operator $\mathcal{L}_{\alpha}$ is linear,

$$
\mathcal{L}_{\alpha}:=L_{\alpha}+V
$$

with $L_{\alpha}$ and $V$ defined as

$$
\begin{gathered}
L_{\alpha}:=-\partial_{z}^{2}+\frac{\alpha^{2}}{4} z^{2}-\frac{3 \alpha}{2}-\frac{1}{2} \partial_{\theta}^{2}, \\
V:=-\frac{2 \alpha}{2+\beta(\tau(\sigma)) z^{2}},
\end{gathered}
$$

respectively. The quantity $\mathcal{W}(a, b)$ is a small linear factor of order $\mathcal{O}(\beta)$, namely

$$
\begin{aligned}
\mathcal{W}(a, b):= & -\frac{\lambda^{2}}{\lambda_{1}^{2}} \frac{\left(a+\frac{1}{2}\right)}{2+b(\tau(\sigma)) y^{2}}+\frac{2 \alpha}{2+\beta(\tau(\sigma)) z^{2}} \\
= & {\left[1-\frac{\lambda^{2}}{\lambda_{1}^{2}}\right] \frac{\left(a+\frac{1}{2}\right)}{2+b(\tau(\sigma)) y^{2}}+\frac{2 \alpha-a-\frac{1}{2}}{2+\beta(\tau(\sigma)) z^{2}} } \\
& +\left(a+\frac{1}{2}\right) \frac{z^{2}[b(\tau(\sigma))-\beta(\tau(\sigma))]}{\left(2+b(\tau(\sigma)) y^{2}\right)\left(2+\beta(\tau(\sigma)) z^{2}\right)} \\
& +\left(a+\frac{1}{2}\right) \frac{\left[y^{2}-z^{2}\right] b(\tau(\sigma))}{\left(2+b(\tau(\sigma)) y^{2}\right)\left(2+\beta(\tau(\sigma)) z^{2}\right)} .
\end{aligned}
$$

The function $\mathcal{F}(a, b)$ is a variant of $F(a, b)$ from definition (9.4), namely

$$
\mathcal{F}(a, b):=e^{-\frac{\alpha}{4} z^{2}} e^{\frac{a}{4} y^{2}} \frac{\lambda_{1}}{\lambda} F(a, b) .
$$

The nonlinear terms $\mathcal{N}_{k},(k=1,2,3)$, are defined using (9.5)-(9.7) by

$$
\begin{aligned}
& \mathcal{N}_{1}(a, b, \eta):=\frac{\lambda_{1}}{\lambda} e^{-\frac{\alpha}{4} z^{2}} e^{\frac{a}{4} y^{2}} N_{1}(a, b, \xi), \\
& \mathcal{N}_{2}(a, b, \eta):=\frac{\lambda_{1}}{\lambda} e^{-\frac{\alpha}{4} z^{2}} e^{\frac{a}{4} y^{2}} N_{2}(a, b, \xi), \\
& \mathcal{N}_{3}(a, b, \eta):=\frac{\lambda_{1}}{\lambda} e^{-\frac{\alpha}{4} z^{2}} e^{\frac{a}{4} y^{2}} N_{3}(a, b, \xi),
\end{aligned}
$$

with $\tau$ and $y$ expressed in terms of $\sigma$ and $z$. Note that $\mathcal{N}_{3}$ was not needed in [9].

Analysis of equation (11.2) will provide estimates for $\eta$, but what we actually need are estimates for $\xi$. To provide this link, we prove in the next proposition that the new trajectory is a good approximation of the old one. 
Proposition 11.1. For any $\tau \leq T$, if $A(\tau) \lesssim \beta^{-\frac{1}{10}}(\tau)$, then

$$
\left|\frac{\lambda}{\lambda_{1}}(t(\tau))-1\right| \leq c \beta(\tau)
$$

for some constant $c$ independent of $\tau$. For $(m, n)=(3,0),(11 / 10,0),(2,1),(1,1)$, one has

$$
\left\|e^{\frac{\alpha}{4} z^{2}} \eta(\cdot, \cdot, \sigma)\right\|_{m, n} \lesssim \beta^{\frac{m+n}{2}+\frac{1}{10}}(\tau(\sigma)) M_{m, n}(\tau(\sigma)) .
$$

Proof. We start by deriving a convenient expression for $\frac{\lambda}{\lambda_{1}}(t(\tau))-1$. By properties of $\lambda$ and $\lambda_{1}$, we have

$$
\partial_{\tau}\left(\frac{\lambda}{\lambda_{1}}(t(\tau))-1\right)=2 a(\tau)\left(\frac{\lambda}{\lambda_{1}}(t(\tau))-1\right)+G(\tau),
$$

where

$$
G:=\alpha-a+(\alpha-a)\left(\frac{\lambda}{\lambda_{1}}-1\right)\left[\left(\frac{\lambda}{\lambda_{1}}\right)^{2}+\frac{\lambda}{\lambda_{1}}+1\right]+a\left(\frac{\lambda}{\lambda_{1}}-1\right)^{2}\left[\frac{\lambda}{\lambda_{1}}+2\right] .
$$

We use the fact that $\frac{\lambda}{\lambda_{1}}(t(\tau))-1=0$ when $\tau=T$ to rewrite equation (11.8) as

$$
\frac{\lambda_{1}}{\lambda}(t(\tau))-1=-\int_{\tau}^{T} e^{-\int_{\tau}^{s} 2 a(t) \mathrm{d} t} G(s) \mathrm{d} s .
$$

In what follows, we rely on equation (11.9) to prove estimate (11.6).

We start by analyzing the integrand on the RHS. The definition of $A(\tau)$ and the condition $A(\tau) \lesssim \beta^{-\frac{1}{20}}(\tau)$ together imply that

$$
\left|a(\tau)-\frac{1}{2}\right| \lesssim \beta(\tau)
$$

and hence that

$$
|a(\tau)-\alpha| \leq\left|a(\tau)-\frac{1}{2}\right|+\left|\alpha-\frac{1}{2}\right| \leq \beta(\tau)
$$

in the time interval $\tau \in[0, T]$. Thus

$$
|G| \lesssim \beta+\left(\frac{\lambda}{\lambda_{1}}-1\right)^{2}+\left|\frac{\lambda}{\lambda_{1}}-1\right|^{3}+\beta\left|\frac{\lambda}{\lambda_{1}}-1\right| .
$$

We claim that inequalities (11.10)-(11.11) are sufficient to prove estimate (11.6). Indeed, define an estimating function $\Lambda(\tau)$ by

$$
\Lambda(\tau):=\sup _{\tau \leq s \leq T} \beta^{-1}(s)\left|\frac{\lambda}{\lambda_{1}}(t(s))-1\right| .
$$

Then (11.9) and the conditions $A(\tau), B(\tau) \lesssim \beta^{-\frac{1}{20}}(\tau)$ imply that $2 a \geq \frac{1}{2}$ and hence that

$$
\begin{aligned}
\left|\frac{\lambda}{\lambda_{1}}(t(\tau))-1\right| & \lesssim \int_{\tau}^{T} e^{-\frac{1}{2}(T-\tau)}\left[\beta(s)+\beta^{2}(s) \Lambda^{2}(\tau)+\beta^{2}(s) \Lambda(\tau)\right] \mathrm{d} s \\
& \lesssim \beta(\tau)+\beta^{2}(\tau) \Lambda^{2}(\tau)+\beta^{3}(\tau) \Lambda^{3}(\tau)+\beta^{2}(\tau) \Lambda(\tau) .
\end{aligned}
$$

Therefore,

$$
\beta^{-1}(\tau)\left|\frac{\lambda}{\lambda_{1}}(t(\tau))-1\right| \lesssim 1+\beta(\tau) \Lambda^{2}(\tau)+\beta^{2}(\tau) \Lambda^{3}(\tau)+\beta(\tau) \Lambda(\tau)
$$


Now we use the facts that $\beta(\tau)$ and $\Lambda(\tau)$ are decreasing functions to obtain

$$
\Lambda(\tau) \lesssim 1+\beta(\tau) \Lambda^{2}(\tau)+\beta^{2}(\tau) \Lambda^{3}(\tau)+\beta(\tau) \Lambda(\tau),
$$

which together with $\Lambda(T)=0$ implies that $\Lambda(\tau) \lesssim 1$ for any time $\tau \in[0, T]$. Combining this with the definition of $\Lambda(\tau)$ gives estimate (11.6).

In the rest of this section, we study the linear operator $\mathcal{L}_{\alpha}$. By Lemma 2.1, it suffices to consider its spectrum acting on $\left\{w: \mathbb{R} \times \mathbb{S}^{1}: w(z, \theta)=w(z, \theta+\pi)\right\}$. Due to the presence of the quadratic term $\frac{1}{4} \alpha z^{2}$, the operator $\mathcal{L}_{\alpha}$ has a discrete spectrum. For $\beta z^{2} \ll 1$, it is close to the harmonic oscillator Hamiltonian

$$
L_{\alpha}-\alpha:=-\partial_{z}^{2}+\frac{1}{4} \alpha^{2} z^{2}-\frac{5 \alpha}{2}-\frac{1}{2} \partial_{\theta}^{2} .
$$

The spectrum of the operator $L_{\alpha}-\alpha$ is

$$
\operatorname{spec}\left(L_{\alpha}-\alpha\right)=\left\{n \alpha+2 k^{2}: n=-2,-1,0,1, \ldots ; k=0,1,2, \ldots\right\} .
$$

Thus it is essential that we can solve the evolution equation (11.2) on the subspace orthogonal to the first three eigenvectors of $L_{\alpha}$. These eigenvectors, normalized, are

$$
\begin{aligned}
& \phi_{0, \alpha}(z):=\left(\frac{\alpha}{2 \pi}\right)^{\frac{1}{4}} e^{-\frac{\alpha}{4} z^{2}}, \\
& \phi_{1, \alpha}(z):=\left(\frac{\alpha}{2 \pi}\right)^{\frac{1}{4}} \sqrt{\alpha} z e^{-\frac{\alpha}{4} z^{2}}, \\
& \phi_{2, \alpha}(z):=\left(\frac{\alpha}{8 \pi}\right)^{\frac{1}{4}}\left(1-\alpha z^{2}\right) e^{-\frac{\alpha}{4} z^{2}} .
\end{aligned}
$$

We define the orthogonal projection $\bar{P}_{n}^{\alpha}$ onto the space spanned by the first $n$ eigenvectors of $L_{\alpha}$ by

$$
\bar{P}_{n}^{\alpha} w:=\sum_{m=0}^{n-1}\left\langle w, \phi_{m, \alpha}\right\rangle,
$$

and the orthogonal projection

$$
P_{n}^{\alpha} w:=1-\bar{P}_{n}^{\alpha} w, \quad(n=1,2,3) .
$$

The following proposition provides useful decay estimates for the propagators generated by $-L_{\alpha}$ and $-\mathcal{L}_{\alpha}$.

Proposition 11.2. If $g: \mathbb{R} \times \mathbb{S}^{1} \rightarrow \mathbb{R}$ is a function satisfying $g(y, \theta)=g(y, \theta+\pi)$ for all $y \in \mathbb{R}$ and $\theta \in \mathbb{S}^{1}$, then for any times $\tau \geq \sigma \geq 0$, one has

$$
\left\|\langle z\rangle^{-n} e^{\frac{\alpha}{4} z^{2}} e^{-L_{\alpha} \sigma} P_{2}^{\alpha} g\right\|_{\infty} \lesssim e^{(1-n) \alpha \sigma}\left\|\langle z\rangle^{-n} e^{\frac{\alpha}{4} z^{2}} g\right\|_{\infty}
$$

with $2 \geq n \geq 1$. Moreover,

$$
\left\|\langle z\rangle^{-1} e^{\frac{\alpha}{4} z^{2}} e^{-L_{\alpha} \sigma} P_{1}^{\alpha} g\right\|_{\infty} \lesssim\left\|\langle z\rangle^{-1} e^{\frac{\alpha}{4} z^{2}} g\right\|_{\infty} ;
$$

and there exist constants $\gamma, \delta>0$ such that if $\beta(0) \leq \delta$, then

$$
\left\|\langle z\rangle^{-n} e^{\frac{\alpha}{4} z^{2}} P_{n}^{\alpha} U_{n}(\tau, \sigma) e^{(\tau-\sigma) \frac{1}{2} \partial_{\theta}^{2}} P_{n}^{\alpha} g\right\|_{\infty} \lesssim e^{-(\gamma+(n-3) \alpha)(\tau-\sigma)}\left\|\langle z\rangle^{-n} e^{\frac{\alpha}{4} z^{2}} g\right\|_{\infty},
$$

where $U_{n}(\tau, \sigma)$ denotes the propagator generated by the operator $-P_{n}^{\alpha}\left[\mathcal{L}_{\alpha}+\frac{1}{2} \partial_{\theta}^{2}\right]_{n}^{\alpha}$, $(n=1,2,3)$. 
Proof. What makes estimates (11.17)-(11.19) different from the corresponding results in the earlier paper [9] is the presence of the operator $\partial_{\theta}^{2}$, which has discrete spectrum $\{0,4,8, \cdots\}$ in the space $\left\{h: \mathbb{S}^{1} \rightarrow \mathbb{R}: h(\theta)=h(\theta+\pi)\right\}$. (Compare Remark 4.3.)

The general strategy in the present situation is to decompose the function $g$ : $\mathbb{R} \times \mathbb{S}^{1} \rightarrow \mathbb{R}$ according to the spectrum of $\partial_{\theta}^{2}$. One then obtains the desired estimate by studying the terms of this decomposition. If a real-valued function $g$ satisfies $g(y, \theta)=g(y, \theta+\pi)$, then it admits the decomposition

$$
g(y, \theta)=\sum_{k=-\infty}^{\infty} e^{2 i k \theta} g_{k}(y)
$$

with $g_{k}(y):=\frac{1}{2 \pi} \int_{0}^{2 \pi} e^{-2 i k \theta} g(y, \theta) \mathrm{d} \theta$. By the definition of $P_{n}$ in equation (11.16), one has $\left(1-P_{n}\right) e^{2 i k \theta} g_{k}(y)=0$ if $k \neq 0$, and hence

$$
P_{n} g=P_{n} g_{0}+\sum_{k \neq 0} e^{2 i k \theta} g_{k} .
$$

Moreover,

$$
e^{\frac{1}{2} \sigma \partial_{\theta}^{2}} P_{n} g=P_{n} g_{0}+e^{\frac{1}{2} \sigma \partial_{\theta}^{2}} \sum_{k \neq 0} e^{2 i k \theta} g_{k} .
$$

Now apply these observations to the propagator acting on $g$ to obtain

$$
\begin{aligned}
& \langle z\rangle^{-n} e^{\frac{\alpha}{4} z^{2}} P_{n}^{\alpha} U_{n}(\tau, \sigma) e^{(\tau-\sigma) \frac{1}{2} \partial_{\theta}^{2}} P_{n}^{\alpha} g \\
& =\langle z\rangle^{-n} e^{\frac{\alpha}{4} z^{2}} P_{n}^{\alpha} U_{n}(\tau, \sigma) P_{n}^{\alpha} g_{0} \\
& \quad+\langle z\rangle^{-n} e^{\frac{\alpha}{4} z^{2}} \tilde{U}(\tau, \sigma) e^{(\tau-\sigma) \frac{1}{2} \partial_{\theta}^{2}} \sum_{k \neq 0} e^{2 i k \theta} g_{k},
\end{aligned}
$$

where $\tilde{U}$ is generated by the operator

$$
-\left[\mathcal{L}_{\alpha}+\frac{1}{2} \partial_{\theta}^{2}\right]=-\left[-\partial_{z}^{2}+\frac{\alpha^{2}}{4} z^{2}-\frac{3 \alpha}{2}-\frac{2 \alpha}{2+\beta(\tau(\sigma)) z^{2}}\right] .
$$

For the first term on the RHS, we apply results proved in [9] to obtain

$$
\left\|\langle z\rangle^{-n} e^{\frac{\alpha}{4} z^{2}} P_{n}^{\alpha} U_{n}(\tau, \sigma) P_{n}^{\alpha} g_{0}\right\|_{\infty} \lesssim e^{-(\gamma+(n-3) \alpha)(\tau-\sigma)}\left\|\langle z\rangle^{-n} e^{\frac{\alpha}{4} z^{2}} g_{0}\right\|_{\infty} .
$$

For the second term on the RHS, we apply the Trotter formula to see that

$$
\tilde{U}(\tau, \sigma) \leq e^{-(\tau-\sigma)\left(-\partial_{z}^{2}+\frac{1}{4} \alpha^{2} z^{2}-\frac{5}{2} \alpha\right)} .
$$

The latter harmonic oscillator be bounded by the non-decaying estimate $e^{2 \alpha(\tau-\sigma)}$ in suitable normed spaces (for details see $[7,9]$ ). Thus one has

$$
\begin{aligned}
& \left\|\langle z\rangle^{-n} e^{\frac{\alpha}{4} z^{2}} \tilde{U}(\tau, \sigma) e^{(\tau-\sigma) \frac{1}{2} \partial_{\theta}^{2}} \sum_{k \neq 0} e^{2 i k \theta} g_{k}\right\|_{\infty} \\
& \leq e^{2 \alpha(\tau-\sigma)}\left\|\langle z\rangle^{-n} e^{\frac{\alpha}{4} z^{2}} e^{(\tau-\sigma) \frac{1}{2} \partial_{\theta}^{2}} \sum_{k \neq 0} e^{2 i k \theta} g_{k}\right\|_{\infty} .
\end{aligned}
$$


For the last term above, one may apply the maximum principle to see that

$$
\left|e^{\frac{1}{2} \sigma \partial_{\theta}^{2}} \sum_{k \neq 0} e^{2 i k \theta} g_{k}\right| \leq \max _{\theta \in[0,2 \pi)}\left|\sum_{k \neq 0} e^{2 i k \theta} g_{k}\right| \leq 2 \max _{\theta \in[0,2 \pi)}|g|
$$

for any $\sigma \geq 0 ;$ and for $\sigma \geq 1$

$$
\begin{aligned}
\left|e^{\frac{1}{2} \sigma \partial_{\theta}^{2}} \sum_{k \neq 0} e^{2 i k \theta} g_{k}\right| & =\left|\sum_{k \neq 0} e^{-2 \sigma k^{2}} e^{2 i k \theta} g_{k}\right| \\
& \lesssim e^{-2 \sigma} \max _{k}\left|g_{k}\right| \\
& =e^{-2 \sigma} \frac{1}{2 \pi} \max _{k}\left|\left\langle g, e^{2 k i \theta}\right\rangle_{\theta}\right| \\
& \leq e^{-2 \sigma} \max _{\theta}|g| .
\end{aligned}
$$

Collecting the estimates above, we conclude that

$$
\left\|\langle z\rangle^{-n} e^{\frac{\alpha}{4} z^{2}} \tilde{U}(\tau, \sigma) e^{(\tau-\sigma) \frac{1}{2} \partial_{\theta}^{2}} \sum_{k \neq 0} e^{2 i k \theta} g_{k}\right\|_{\infty} \lesssim e^{-(\tau-\sigma)}\left\|\langle z\rangle^{-n} e^{\frac{\alpha}{4} z^{2}} g\right\|_{\infty} .
$$

This together with equation (11.20) and estimate (11.21) implies inequality (11.19).

The proofs of estimates (11.17)-(11.18) are similar modifications of those detailed in $[7,9]$, hence are omitted here.

11.1. Proof of estimate (9.12). In this section, we prove estimate (9.12) for the function $M_{3,0}$. Fix a ( $\tau$-scale) time $T$. Then the estimates of Proposition 11.1 hold for $\tau \leq T$. We start by estimating $\eta$ defined in equation (11.1). We observe that $\eta$ is not orthogonal to the first three eigenvectors of the operator $L_{\alpha}$. Therefore, we derive an equation for $P_{3}^{\alpha} \eta$, namely

$$
\partial_{\sigma}\left(P_{3}^{\alpha} \eta\right)=-P_{3}^{\alpha} \mathcal{L}_{\alpha} P_{3}^{\alpha} \eta+\sum_{k=1}^{6} D_{3,0}^{(k)}(\sigma)
$$

where $D_{m, n}^{(k)} \equiv D_{m, n}^{(k)}(\sigma)$, with $k=1,2,3,4,5,6,(m, n)=(3,0),(2,0),(2,1),(1,1)$, are defined as ${ }^{9}$

$$
\begin{aligned}
D_{m, n}^{(1)} & :=-P_{m}^{\alpha} V e^{-\frac{\alpha}{4} z^{2}} \partial_{z}^{n}\left[e^{\frac{\alpha}{4} z^{2}} \eta\right]+P_{m}^{\alpha} V P_{m}^{\alpha} e^{-\frac{\alpha}{4} z^{2}} \partial_{z}^{n}\left[e^{\frac{\alpha}{4} z^{2}} \eta\right] \\
& =-P_{m}^{\alpha} V e^{-\frac{\alpha}{4} z^{2}}\left[1-P_{m}^{\alpha}\right] \partial_{z}^{2}\left[e^{\frac{\alpha}{4} z^{2}} \eta\right] \\
& =P_{m}^{\alpha} \frac{\alpha \beta(\tau(\sigma)) z^{2}}{2+\beta(\tau(\sigma)) z^{2}}\left[1-P_{m}^{\alpha}\right] e^{-\frac{\alpha}{4} z^{2}} \partial_{z}^{n}\left[e^{\frac{\alpha}{4} z^{2}} \eta\right],
\end{aligned}
$$

and

$$
\begin{aligned}
& D_{m, n}^{(2)}:=P_{m}^{\alpha} e^{-\frac{\alpha}{4} z^{2}} \partial_{z}^{n}\left[e^{\frac{\alpha}{4} z^{2}} \mathcal{W} \eta\right], \\
& D_{m, n}^{(3)}:=P_{m}^{\alpha} e^{-\frac{\alpha}{4} z^{2}} \partial_{z}^{n}\left[e^{\frac{\alpha}{4} z^{2}} \mathcal{F}(a, b)\right], \\
& D_{m, n}^{(4)}:=P_{m}^{\alpha} e^{-\frac{\alpha}{4} z^{2}} \partial_{z}^{n}\left[e^{\frac{\alpha}{4} z^{2}} \mathcal{N}_{1}(a, b, \alpha, \eta)\right], \\
& D_{m, n}^{(5)}:=P_{m}^{\alpha} e^{-\frac{\alpha}{4} z^{2}} \partial_{z}^{n}\left[e^{\frac{\alpha}{4} z^{2}} \mathcal{N}_{2}\right], \\
& D_{m, n}^{(6)}:=P_{m}^{\alpha} e^{-\frac{\alpha}{4} z^{2}} \partial_{z}^{n}\left[e^{\frac{\alpha}{4} z^{2}} \mathcal{N}_{3}\right] .
\end{aligned}
$$

${ }^{9}$ Recall definitions (11.3)-(11.5) and (11.16). 
Define

$$
S:=\int_{0}^{t(T)} \lambda_{1}^{-2}(s) \mathrm{d} s
$$

Then by Duhamel's principle, we may rewrite equation (11.26) as

$$
P_{3}^{\alpha} \eta(S)=P_{3}^{\alpha} U_{3}(S, 0) P_{3}^{\alpha} \eta(0)+\sum_{n=1}^{6} \int_{0}^{S} P_{3}^{\alpha} U_{3}(S, \sigma) P_{3}^{\alpha} D_{3,0}^{(n)}(\sigma) \mathrm{d} \sigma,
$$

where $U_{3}(\tau, \sigma)$ is defined in Proposition 11.2 and satisfies estimate (11.19). It follows that

$$
\begin{aligned}
& \beta^{-\frac{8}{5}}(T)\left\|e^{\frac{\alpha}{4} z^{2}} P_{3}^{\alpha} \eta(S)\right\|_{3,0} \\
& \lesssim e^{-\gamma S} \beta^{-\frac{8}{5}}(T)\left\|e^{\frac{\alpha}{4} z^{2}} \eta(0)\right\|_{3,0} \\
& \quad+\beta^{-\frac{8}{5}}(T) \sum_{k=1}^{6} \int_{0}^{S} e^{-\gamma(S-\sigma)}\left\|e^{\frac{\alpha}{4} z^{2}} D_{3,0}^{(k)}(\sigma)\right\|_{3,0} \mathrm{~d} \sigma .
\end{aligned}
$$

For the terms $D_{3,0}^{(k)}$ with $k=1,2,3,4,5$, on the RHS of equation (11.26), one has the following estimates.

Lemma 11.3. If $A(\tau), B(\tau) \lesssim \beta^{-\frac{1}{20}}(\tau)$ and if $\sigma \leq S$, equivalently $\tau \leq T$, then

$$
\begin{aligned}
& \sum_{k=1}^{3}\left\|e^{\frac{\alpha}{4} z^{2}} P_{3}^{\alpha} D_{3,0}^{(k)}(\sigma)\right\|_{3,0} \lesssim \beta^{\frac{33}{20}}(\tau(\sigma)) P(M(T), A(T)), \\
& \left\|e^{\frac{\alpha}{4} z^{2}} P_{3}^{\alpha} D_{3,0}^{(4)}(\sigma)\right\|_{3,0} \lesssim\left\|e^{\frac{a y^{2}}{4}} N_{1}(a, b, \xi)\right\|_{3,0} \lesssim \beta^{\frac{33}{20}}(\tau) P(M(\tau)), \\
& \left\|e^{\frac{\alpha}{4} z^{2}} P_{3}^{\alpha} D_{3,0}^{(5)}(\sigma)\right\|_{3,0} \lesssim\left\|e^{\frac{a y^{2}}{4}} N_{2}(a, b, \xi)\right\|_{3,0} \lesssim \beta^{\frac{33}{20}}(\tau) P(M(\tau)),
\end{aligned}
$$

and

$$
\left\|e^{\frac{\alpha}{4} z^{2}} P_{3}^{\alpha} D_{3,0}^{(6)}(\sigma)\right\|_{3,0} \lesssim\left\|e^{\frac{a y^{2}}{4}} N_{3}(a, b, \xi)\right\|_{3,0} \lesssim \beta^{\frac{33}{20}}(\tau) .
$$

Proof. We first prove the most involved estimate, namely (11.33). Its first inequality is a consequence of the fact that $\frac{\lambda_{1}}{\lambda}-1=\mathcal{O}(\beta)$ proved in estimate (11.6). Because estimates of this type will appear many times below, we provide a detailed proof (only) here. Unwrapping the definitions of the functions involved, one finds that

$$
\begin{aligned}
\left\|e^{\frac{\alpha}{4} z^{2}} P_{3}^{\alpha} D_{3,0}^{(6)}(\sigma)\right\|_{3,0} & \leq\left\|e^{\frac{\alpha}{4} z^{2}} D_{3,0}^{(6)}(\sigma)\right\|_{3,0} \\
& \leq\left\|\langle z\rangle^{-3} e^{\frac{a}{4} y^{2}} N_{3}(a, b, \xi)(\tau(\sigma))\right\|_{\infty} \\
& \lesssim\left\|\langle y\rangle^{-3} e^{\frac{a}{4} y^{2}} N_{3}(a, b, \xi)(\tau(\sigma))\right\|_{\infty} \\
& =\left\|e^{\frac{a}{4} y^{2}} N_{3}(a, b, \xi)\right\|_{3,0} .
\end{aligned}
$$

To prove the second inequality in estimate (11.33), we write the new term $N_{3}$ as

$$
N_{3}:=N_{3,1}+N_{3,2}
$$

with

$$
N_{3,1}:=\left[v^{-2}-\frac{1}{2}\right]\left(\partial_{\theta}^{2} v\right) e^{-\frac{a y^{2}}{4}}
$$


and

$$
\begin{aligned}
N_{3,2}:=-v^{-2} \frac{q^{2}}{1+} & q^{2}+p^{2} \\
& \left(\partial_{\theta}^{2} v\right) e^{-\frac{a y^{2}}{4}} \\
& +e^{-\frac{a y^{2}}{4}} v^{-1} \frac{2 p q}{1+p^{2}+q^{2}}\left(\partial_{\theta} \partial_{y} v\right)+e^{-\frac{a y^{2}}{4}} v^{-2} \frac{q}{1+p^{2}+q^{2}}\left(\partial_{\theta} v\right) .
\end{aligned}
$$

We shall only prove estimate (11.33) for $N_{3,1}$; the result for $N_{3,2}$ is easier, when one recalls from estimate (8.11) that $v^{-2} \partial_{\theta}^{2} v, v^{-1} \partial_{\theta} \partial_{y} v=\mathcal{O}\left(\beta^{\frac{33}{20}}\right)$. For the term $v^{-2} \partial_{\theta} v$ above, we apply the interpolation result in Lemma B.2 to inequality (8.11) to get

$$
\left|v^{-2} \partial_{\theta} v\right| \lesssim \max _{\theta \in[0,2 \pi]}\left|v^{-2} \partial_{\theta}^{2} v\right| \lesssim \beta^{\frac{33}{20}}
$$

Then by estimate (4.7) that $\langle y\rangle^{-1} v \lesssim 1$ and estimate (8.11), we obtain

$$
\langle y\rangle^{-3}\left[v^{-2}-\frac{1}{2}\right]\left|\partial_{\theta}^{2} v\right| \lesssim v^{-2}\left|\partial_{\theta}^{2} v\right| \lesssim \beta^{\frac{33}{20}},
$$

which gives the desired result.

Now consider inequality (11.30). For $k=1$, we use the identity $P_{3}^{\alpha}\left(1-P_{3}^{\alpha}\right)=0$ to rewrite $P_{3}^{\alpha} D_{3,0}^{(1)}$ as

$$
P_{3}^{\alpha} D_{3,0}^{(1)}(\sigma)=P_{3}^{\alpha} \frac{\alpha+\frac{1}{2}}{2+b(\tau(\sigma)) z^{2}} b(\tau(\sigma)) z^{2}\left(1-P_{3}^{\alpha}\right) \eta(\sigma) .
$$

Then direct computation gives the desired estimate,

$$
\begin{aligned}
\left\|e^{\frac{\alpha}{4} z^{2}} P_{3}^{\alpha} D_{3,0}^{(1)}(\sigma)\right\|_{3,0} & \lesssim \max _{z \in \mathbb{R}}\left|\langle z\rangle^{-1} \frac{b(\tau(\sigma)) z^{2}}{1+b z^{2}}\right|\left|\langle z\rangle^{-2} e^{\frac{\alpha}{4} z^{2}}\left(1-P_{3}^{\alpha}\right) \eta(\sigma)\right| \\
& \lesssim b^{\frac{1}{2}}(\tau(\sigma))\left\|e^{\frac{\alpha}{4} z^{2}} \eta(\sigma)\right\|_{3,0} \\
& \lesssim \beta^{2}(\tau(\sigma)) M_{3,0}(T) .
\end{aligned}
$$

Here we used the fact that $|b(\tau)| \leq 2 \beta(\tau)$, which is implied by $B(\tau) \leq \beta^{-\frac{1}{10}}(\tau)$, and the fact for any $m \geq 1$, one has

$$
\left\|e^{\frac{\alpha}{4} z^{2}}\left(1-P_{m}^{\alpha}\right) g\right\|_{m-1,0} \lesssim\left\|e^{\frac{\alpha}{4} z^{2}} g\right\|_{m, 0}
$$

by definition (11.15) of the orthogonal projections $\bar{P}_{n}^{\alpha} \equiv 1-P_{m}^{\alpha}$.

Now we estimate $D_{3,0}^{(k)}$ for $k=2,3$. By estimate (11.37) and direct computation, we have

$$
\sum_{k=2}^{5}\left\|e^{\frac{\alpha}{4} z^{2}} P_{3}^{\alpha} D_{3,0}^{(k)}(\sigma)\right\|_{3,0} \leq\left\|e^{\frac{\alpha}{4} z^{2}} \mathcal{F}(a, b)(\sigma)\right\|_{3,0}+\left\|e^{\frac{\alpha}{4} z^{2}} \mathcal{W} \eta(\sigma)\right\|_{3,0} .
$$

By estimate (11.6) that $\frac{\lambda_{1}}{\lambda}-1=\mathcal{O}(\beta)$, one then obtains

$$
\left\|e^{\frac{\alpha}{4} z^{2}} \mathcal{F}(\sigma)\right\|_{3,0} \lesssim\|F(a, b)(\tau(\sigma))\|_{3,0} \leq \beta^{2} P(M(T), A(T)),
$$

where $F(a, b)$ is defined in equation (9.4) and estimated in (10.8). Recalling definition (11.3) for $\mathcal{W}=\mathcal{O}(\beta)$, one has

$$
\left\|e^{\frac{\alpha}{4} z^{2}} \mathcal{W} \eta\right\|_{3,0} \lesssim\|\mathcal{W}\|_{\infty}\left\|e^{\frac{\alpha}{4} z^{2}} \eta\right\|_{3,0} \lesssim \beta^{\frac{13}{5}} M_{3,0}
$$

The first inequalities in estimates (11.31)-(11.32) are derived in almost exactly the same way as estimate (11.34); so we omit further details here. 
The proof of the second inequality in estimate (11.31) makes it necessary to prove a bound for $\left\|\langle y\rangle^{\frac{11}{10}} e^{\frac{a}{4} y^{2}} \xi\right\|_{\infty}$. Specifically,

$$
\begin{aligned}
\left\|\langle z\rangle^{-3} D_{3,0}^{(4)}(\sigma)\right\|_{\infty} & \lesssim\left\|\langle y\rangle^{-3} e^{\frac{a}{4} y^{2}} N_{1}(\tau(\sigma))\right\|_{\infty} \\
& \lesssim\left\|\langle y\rangle^{-3} e^{\frac{a}{2} y^{2}} \frac{1}{2+b y^{2}} \xi^{2}\right\|_{\infty} \\
& \lesssim\left\|\langle y\rangle^{-3} e^{\frac{a}{4} y^{2}} \xi\right\|_{\infty}\left\|\frac{1}{2+b y^{2}} e^{\frac{a}{4} y^{2}} \xi\right\|_{\infty} .
\end{aligned}
$$

By the fact that $\frac{1}{1+b y^{2}} \lesssim \beta^{-\frac{11}{20}}\langle y\rangle^{-\frac{11}{10}}$ and the definitions of $M_{3,0}$ and $M_{11 / 10,0}$, we obtain the desired estimate (11.31).

Next we turn to the second inequality in (11.32). Compute directly to obtain

$$
\left|\langle y\rangle^{-3} e^{\frac{a}{4} y^{2}} N_{2}(a, b, \xi)\right| \lesssim\left|\partial_{y}^{2} v\right|\left|\langle y\rangle^{-1} \partial_{y} v\right|^{2}
$$

By estimate (8.10), the first term on the RHS is bounded by $\left|\partial_{y}^{2} v\right| \lesssim \beta^{\frac{13}{20}}$. For the second term, we recall that $v=\sqrt{2+b y^{2} / a+\frac{1}{2}}+e^{\frac{a y^{2}}{4}} \xi$. So by direct computation, one has

$$
\begin{aligned}
\left|\langle y\rangle^{-1} \partial_{y} v\right| & \lesssim b+\langle y\rangle^{-1}\left|\partial_{y} e^{\frac{a y^{2}}{4}} \xi\right| \\
& \leq b+b^{\frac{11}{10}} M_{1,1} \\
& \lesssim \beta\left(1+M_{1,1}\right) .
\end{aligned}
$$

Feed these estimates into inequality (11.38) to obtain (11.32).

Collecting the estimates above completes the proof of the lemma.

Now we resume our study of equation (11.28). For its first term, we use the slow decay of $\beta(\tau)$ and estimate (11.7) to get

$$
e^{-\gamma S} \beta^{-\frac{8}{5}}(T)\left\|e^{\frac{\alpha}{4} z^{2}} \eta(0)\right\|_{3,0} \lesssim \beta^{-\frac{8}{5}}(0)\left\|e^{\frac{\alpha}{4} z^{2}} \eta(0)\right\|_{3,0} \lesssim M_{3,0}(0) .
$$

For its second term, we use the bounds for $D_{3,0}^{(k)}$ from Lemma 11.3 and the integral estimate (11.41) proved in Lemma 11.4 below to obtain

$$
\begin{aligned}
& \sum_{k=1}^{6} \int_{0}^{S} e^{-\gamma(S-\sigma)}\left\|e^{\frac{\alpha}{4} z^{2}} D_{3,0}^{(k)}(\sigma)\right\|_{3,0} \mathrm{~d} \sigma \\
& \quad \lesssim\left[\int_{0}^{S} e^{-\gamma(S-\sigma)} \beta^{\frac{17}{10}}(\sigma)(\sigma) \mathrm{d} \sigma\right] P(M(T), A(T)) \\
& \quad \lesssim \beta^{\frac{17}{10}}(T) P(M(T), A(T)) .
\end{aligned}
$$

By estimate (11.45), proved in Lemma 11.5 below, we have $\left\|e^{\frac{\alpha}{4} z^{2}} P_{3}^{\alpha} \eta(S)\right\|_{3,0}=$ $\|\phi(\cdot, T)\|_{3,0}$, which together with (11.39) and (11.40) implies that

$$
\beta^{-\frac{8}{5}}(T)\|\phi(\cdot, T)\|_{3,0} \lesssim M_{3,0}(0)+\beta^{\frac{1}{10}}(T) P(M(T), A(T))
$$

where $P$ is a nondecreasing polynomial. By definition (8.2) of $M_{3,0}$, we obtain

$$
M_{3,0}(T) \lesssim M_{3,0}(0)+\beta^{\frac{1}{10}}(0) P(M(T), A(T))
$$


Since $T$ was arbitrary, estimate (9.12) will follow, once we provide a proof of inequalities (11.41) and (11.45) used above.

Here is the first of the two promised lemmas.

Lemma 11.4. For any $c_{1}, c_{2}>0$, there exists a constant $c\left(c_{1}, c_{2}\right)$ such that

$$
\int_{0}^{S} e^{-c_{1}(S-s)} \beta^{c_{2}}(\tau(s)) \mathrm{d} s \leq c\left(c_{1}, c_{2}\right) \beta^{c_{2}}(T) .
$$

Proof. The estimate is based on a simpler observation: there exists $C\left(c_{1}, c_{2}\right)$ such that for any $S \geq 0$, one has

$$
\int_{0}^{S} \frac{e^{-c_{1}(S-s)}}{(1+s)^{c_{2}}} \mathrm{~d} s \leq \frac{C\left(c_{1}, c_{2}\right)}{(1+S)^{c_{2}}} .
$$

This is proved by separately considering the regions $s \in\left[0, \frac{S}{2}\right]$ and $s \in\left[\frac{S}{2}, S\right]$, and comparing the sizes of $e^{-c_{1}(S-s)}$ and $\frac{1}{(1+s)^{c_{2}}}$ there.

The proof of inequality (11.41) is complicated by the presence of the two time scales $\sigma$ and $\tau$, which are related by $\sigma(t):=\int_{0}^{t} \lambda_{1}^{-2}(s) \mathrm{d} s$ and $\tau=\int_{0}^{t} \lambda^{-2}(s) \mathrm{d} s$. In what follows, we prove the desired estimate by comparing these two time scales.

By estimate (11.6), one has $\frac{\lambda(\tau)}{\lambda_{1}(\tau(\sigma))} \approx 1$ and thus

$$
4 \sigma \geq \tau(\sigma) \geq \frac{1}{4} \sigma
$$

This implies that $\frac{1}{\frac{1}{b_{0}}+\tau(\sigma)} \leq 4 \frac{1}{\frac{1}{b_{0}}+\sigma}$, which in turn gives

$$
\int_{0}^{S} e^{-c_{1}(S-s)} \beta^{c_{2}}(\tau(s)) \mathrm{d} s \leq \frac{c\left(c_{1}, c_{2}\right)}{\left(\frac{1}{b_{0}}+S\right)^{c_{2}}} .
$$

Recall that $\tau(S)=T$ and use estimate (11.43) again to obtain

$$
\frac{1}{\left(\frac{1}{b_{0}}+S\right)^{c_{2}}} \lesssim \frac{1}{\left(\frac{1}{b_{0}}+T\right)^{c_{2}}}
$$

This together with inequality (11.44) implies the desired estimate.

The next lemma relates $\phi:=e^{\frac{a y^{2}}{4}} \xi$ and $\eta$ at times $\sigma=S$ and $\tau=T$.

Lemma 11.5. If $m+n \leq 3$ and $\ell \geq 0$, then

$$
\langle z\rangle^{-\ell} e^{\frac{\alpha z^{2}}{4}} P_{m}^{\alpha}\left(\partial_{z}+\frac{\alpha}{2} z\right)^{n} \eta(z, S)=\langle y\rangle^{-\ell} \partial_{y}^{n} \phi(y, T) .
$$

Proof. By definition of the various quantities in equation (11.1), one has $\lambda_{1}(t(T))=$ $\lambda(t(T)), a(T)=\alpha$, and hence $z=y$ and $e^{\frac{\alpha}{4} z^{2}} \eta(z, S)=\phi(y, T)$. Then because $e^{\frac{\alpha}{4} z^{2}}\left(\partial_{z}+\frac{\alpha}{2} z\right) e^{-\frac{\alpha}{4} z^{2}}=\partial_{z}$, we have

$$
\langle z\rangle^{-\ell} e^{\frac{\alpha z^{2}}{4}} P_{m}^{\alpha}\left(\partial_{z}+\frac{\alpha}{2} z\right)^{n} \eta(z, \theta, S)=\langle y\rangle^{-\ell} e^{\frac{a(T) y^{2}}{4}} P_{m}^{a(T)} e^{-\frac{a(T) y^{2}}{4}} \partial_{y}^{n} \phi(y, \theta, T) .
$$

Integrating by parts and using the orthogonality conditions $\xi(\cdot, \tau) \perp \phi_{k, a(\tau)}$ for $k=0,1,2$, with $\phi_{k, a}$ defined in equation (9.2), one gets

$$
e^{-\frac{a(\tau) y^{2}}{4}} \partial_{y}^{n} \phi(\cdot, \tau) \perp \phi_{k, a(\tau)}, \quad(0 \leq k \leq 2-n),
$$


which shows that

$$
P_{m}^{a(T)} e^{-\frac{a(T) y^{2}}{4}} \partial_{y}^{n} \phi(y, \theta, T)=e^{-\frac{a(T) y^{2}}{4}} \partial_{y}^{n} \phi(y, \theta, T)
$$

if $m+n \leq 3$. This together with equation (11.46) implies identity (11.45).

Our proof of estimate (9.12) is complete.

11.2. Proof of estimate (9.13). In this section, we prove estimate (9.13) for the function $M_{11 / 10,0}$. To begin, we use equation (11.2) to compute that $P_{2}^{\alpha} \eta(\sigma)$ evolves by

$$
\partial_{\sigma}\left(P_{2}^{\alpha} \eta\right)=-L_{\alpha} P_{2}^{\alpha} \eta-P_{2}^{\alpha} V \eta+P_{2}^{\alpha} \sum_{k=2}^{6} D_{2,0}^{(k)}
$$

where the functions $D_{2,0}^{(k)}$ and the operator $L_{\alpha}$ (which differs from $\mathcal{L}_{\alpha}$ by the absence of the potential $V$ ) are defined immediately after equations (11.26) and (11.2), respectively. Our first step is to obtain the following bounds.

Lemma 11.6. If $A(\tau), B(\tau) \lesssim \beta^{-\frac{1}{10}}(\tau)$, then

$$
\begin{gathered}
\left\|e^{\frac{\alpha}{4} z^{2}} V \eta(\sigma)\right\|_{\frac{11}{10}, 0} \lesssim \beta^{\frac{13}{20}}(\tau(\sigma)) M_{3,0}(T), \\
\sum_{k=2}^{5}\left\|e^{\frac{\alpha}{4} z^{2}} P_{2}^{\alpha} D_{2,0}^{(k)}(\sigma)\right\|_{\frac{11}{10}, 0} \lesssim \beta^{\frac{7}{10}}(\tau) P(M(T), A(T)), \\
\left\|e^{\frac{\alpha}{4} z^{2}} P_{2}^{\alpha} D_{2,0}^{(6)}(\sigma)\right\|_{\frac{11}{10}, 0} \lesssim\left\|e^{\frac{a y^{2}}{4}} N_{3}(a, b, \xi)\right\|_{\frac{11}{10}, 0} \lesssim \beta^{\frac{13}{20}}(\tau)\left(1+\epsilon_{0} M_{\frac{11}{10}, 0}\right),
\end{gathered}
$$

where $\epsilon_{0}$ is the small constant that appears in estimate (8.9).

Proof. We start by proving inequality (11.48). By Condition [Cb], we have $\frac{1}{b} \lesssim \frac{1}{\beta}$ and hence

$$
\frac{\langle z\rangle^{-\frac{11}{10}}}{1+b(\tau(\sigma)) z^{2}} \leq\langle z\rangle^{-\frac{11}{10}}\left(1+b(\tau(\sigma)) z^{2}\right)^{-\frac{19}{20}} \lesssim \beta^{-\frac{19}{20}}(\tau(\sigma))\langle z\rangle^{-3},
$$

which, together with estimate (11.7) and the definition of $V$ following equation (11.2), yields

$$
\begin{aligned}
\left\|e^{\frac{\alpha}{4} z^{2}} V \eta(\sigma)\right\|_{\frac{11}{10}, 0} & \lesssim\left\|\frac{1}{1+b(\tau(\sigma)) z^{2}} e^{\frac{\alpha}{4} z^{2}} \eta(\sigma)\right\|_{\frac{11}{10}, 0} \\
& \lesssim \beta^{-\frac{19}{20}}(\tau(\sigma))\left\|e^{\frac{\alpha}{4} z^{2}} \eta(\sigma)\right\|_{3,0} \\
& \lesssim \beta^{\frac{13}{20}}(\tau(\sigma)) M_{3,0}(T) .
\end{aligned}
$$

This gives inequality (11.48).

The proof of inequality (11.49) is almost identical to that of (11.30), thus is omitted here. (It may be compared with the corresponding part of [9].)

Now we turn to inequality (11.50). As in equation (11.35), we decompose the new term $N_{3}$ into two parts, $N_{3}=N_{3,1}+N_{3,2}$. We only prove estimate (11.33) for $N_{3,1}$; the estimate for $N_{3,2}$ are easier and use the sufficiently fast decay estimates for $v^{-2} \partial_{\theta}^{2} v, v^{-1} \partial_{\theta} \partial_{y} v, v^{-2} \partial_{\theta} v=\mathcal{O}\left(\beta^{\frac{33}{20}}\right)$ found in (8.11) and (11.36). 
By direct computation, we find that

$$
\langle y\rangle^{-\frac{11}{10}} e^{\frac{a y^{2}}{4}} N_{3,1}=\frac{1}{2} \frac{\sqrt{2}+v}{v}\langle y\rangle^{-\frac{11}{10}}[\sqrt{2}-v] v^{-1} \partial_{\theta}^{2} v .
$$

The lower bound $v \geq 1$ from (8.7) and condition [Cb] imply that

$$
\left|\langle y\rangle^{-\frac{11}{10}} e^{\frac{a y^{2}}{4}} N_{3,1}\right| \lesssim\langle y\rangle^{-\frac{11}{10}}|\sqrt{2}-v| v^{-1}\left|\partial_{\theta}^{2} v\right| .
$$

For the term $\sqrt{2}-v$, we use the decomposition $v=e^{\frac{a}{4} y^{2}} w$ in equation (9.1) to write

$$
\begin{aligned}
\sqrt{2}-v & =\sqrt{2}-\sqrt{\frac{2+b y^{2}}{a+\frac{1}{2}}}-e^{\frac{a}{4} y^{2} \xi} \\
& =-\left(a+\frac{1}{2}\right)^{-\frac{1}{2}} \frac{b y^{2}}{\sqrt{2}+\sqrt{2+b y^{2}}}+\frac{\sqrt{2}}{\sqrt{a+\frac{1}{2}}} \frac{a-\frac{1}{2}}{1+\sqrt{a+\frac{1}{2}}}-e^{\frac{a}{4} y^{2}} \xi .
\end{aligned}
$$

Substitute this into estimate (11.51) to obtain

$$
\begin{aligned}
\left|\langle y\rangle^{-\frac{11}{10}} e^{\frac{a y^{2}}{4}} N_{3,1}\right| & \lesssim\langle y\rangle^{-\frac{11}{10}}\left[\frac{b y^{2}}{\sqrt{2+b y^{2}}}+|1-2 a|+\left|e^{\frac{a y^{2}}{4}} \xi\right|\right] v^{-1}\left|\partial_{\theta}^{2} v\right| \\
& =: C_{1}+C_{2}+C_{3} .
\end{aligned}
$$

To conclude, we shall estimate the three components on the RHS.

The estimate for $C_{1}$ is the most involved. By direct computation, we obtain

$$
C_{1} \leq b^{\frac{1}{2}}\langle y\rangle^{-\frac{1}{10}} v^{-1}\left|\partial_{\theta}^{2} v\right|
$$

Using the estimates $v^{-1}\left|\partial_{\theta}^{2} v\right| \leq \epsilon_{0}$ from (8.9), $v^{-2}\left|\partial_{\theta}^{2} v\right| \lesssim \beta^{\frac{17}{10}}$ from (8.11), and $\frac{v}{1+|y|} \lesssim 1$ from (4.7), the consequence $\beta \approx b$ of Condition [Cb] for $B$, and Hölder's inequality, we get

$$
C_{1} \lesssim b^{\frac{1}{2}}\left[v^{-2}\left|\partial_{\theta}^{2} v\right|\right]^{\frac{1}{10}}\left[v^{-1}\left|\partial_{\theta}^{2} v\right|\right]^{\frac{9}{10}} \lesssim \epsilon_{0}^{\frac{9}{10}} b^{\frac{133}{200}}
$$

For $C_{2}$, we use the fact $a-\frac{1}{2}=\mathcal{O}(b)+\mathcal{O}\left(\beta^{2} A\right)$ from estimate (8.3) and Condition [Cb] to obtain

$$
C_{2} \lesssim \epsilon_{0} \beta
$$

For $C_{3}$, we use definition (8.2) of $M_{1,1}$ to get

$$
C_{3} \leq \epsilon_{0} M_{1,1} \beta^{\frac{13}{20}}
$$

Collecting inequalities (11.52)-(11.54) completes the estimate for

$$
\left\|\langle y\rangle^{-\frac{11}{10}} e^{\frac{a y^{2}}{4}} N_{3,1}\right\|_{\infty} .
$$

Now fix a ( $\tau$-scale) time $T$. To conclude our proof of estimate (9.13), we continue to study equation (11.47), which may by Duhamel's principle be rewritten in the form

$$
P_{2}^{\alpha} \eta(S)=e^{-L_{\alpha} S} P_{2}^{\alpha} \eta(0)+\int_{0}^{S} e^{-L_{\alpha}(S-\sigma)} P_{2}^{\alpha}\left[-V \eta+\sum_{k=2}^{6} D_{2,0}^{(k)}\right] \mathrm{d} \sigma
$$


where $S=S(t(T))$ is defined in equation (11.27). By estimate (11.17) for $e^{-L_{\alpha} \sigma} P_{2}^{\alpha}$, one has

$$
\left\|e^{\frac{\alpha}{4} z^{2}} P_{2}^{\alpha} \eta(S)\right\|_{\frac{11}{10}, 0} \lesssim K_{0}+K_{1}+K_{2}
$$

where

$$
\begin{aligned}
& K_{0}:=e^{-\alpha S}\left\|e^{\frac{\alpha}{4} z^{2}} \eta(0)\right\|_{\frac{11}{10}, 0}, \\
& K_{1}:=\int_{0}^{S} e^{-\alpha(S-\sigma)}\left\|e^{\frac{\alpha}{4} z^{2}} V \eta(\sigma)\right\|_{\frac{11}{10}, 0} \mathrm{~d} \sigma, \\
& K_{2}:=\sum_{k=2}^{6} \int_{0}^{S} e^{-\alpha(S-\sigma)}\left\|e^{\frac{\alpha}{4} z^{2}} D_{2,0}^{(k)}\right\|_{\frac{11}{10}, 0} \mathrm{~d} \sigma .
\end{aligned}
$$

Next, we estimate the $K_{n}$ for $n=0,1,2$.

(K0) Estimate (11.7) and the slow decay of $\beta$ yield

$$
K_{0} \lesssim \beta^{\frac{13}{20}}(T) \beta^{-\frac{13}{20}}(0)\left\|e^{\frac{\alpha}{4} z^{2}} \eta(0)\right\|_{\frac{11}{10}, 0} \lesssim \beta^{\frac{13}{20}}(T) M_{\frac{11}{10}, 0}(0) .
$$

(K1) Estimate (11.48) and the integral inequality (11.41) imply that

$$
K_{1} \lesssim\left[\int_{0}^{S} e^{-\alpha(S-\sigma)} \beta^{\frac{13}{20}}(\tau(\sigma)) \mathrm{d} \sigma\right] M_{3,0}(T) \lesssim \beta^{\frac{13}{20}}(T) M_{3,0}(T) .
$$

(K2) The estimates of $D_{2,0}^{(k)},(k=2 \ldots 6)$, implied by (11.49) yield the bounds

$$
\begin{aligned}
K_{2} & \lesssim \int_{0}^{S} e^{-\alpha(S-\sigma)}\left[\beta^{\frac{3}{4}}(\tau(\sigma)) P(M(T), A(T))+\beta^{\frac{13}{20}} \epsilon_{0} M_{\frac{11}{10}, 0}(T)\right] \mathrm{d} \sigma \\
& \lesssim \beta^{\frac{3}{4}}(T) P(M(T), A(T))+\beta^{\frac{13}{20}} \epsilon_{0} M_{\frac{11}{10}, 0}(T) .
\end{aligned}
$$

Collecting estimates (11.55)-(11.58), one obtains

$$
\begin{aligned}
& \beta^{-\frac{13}{20}}(T)\left\|e^{\frac{\alpha}{4} z^{2}} P_{2}^{\alpha} \eta(\tau(S))\right\|_{\frac{11}{10}, 0} \\
& \quad \lesssim M_{\frac{11}{10}, 0}(0)+M_{3,0}(T)+\epsilon_{0} M_{\frac{11}{10}, 0}+1+\beta^{\frac{1}{10}}(0) P(M(T), A(T)) .
\end{aligned}
$$

Equation (11.45) shows that

$$
\beta^{-\frac{13}{20}}(T)\|\phi(T)\|_{\frac{11}{10}, 0}=\beta^{-\frac{13}{20}}(T)\left\|e^{\frac{\alpha z^{2}}{4}} P_{2}^{\alpha} \eta(S)\right\|_{\frac{11}{10}, 0},
$$

which together with estimate (11.59) and the definition of $M_{11 / 10,0}$ implies that

$$
M_{\frac{11}{10}, 0}(T) \lesssim M_{\frac{11}{10}, 0}(0)+M_{3,0}(T)+\epsilon_{0} M_{\frac{11}{10}, 0}(\tau)+\beta^{\frac{1}{10}}(0) P(M(T), A(T)) .
$$

Since the time $T$ was arbitrary, the proof of estimate (9.13) is complete. 
11.3. Proof of estimate (9.15). In this section, we prove estimate (9.15) for the function $M_{1,1}$. Observe that for any smooth function $g$, one has

$$
e^{-\frac{\alpha z^{2}}{4}} \partial_{z}\left(e^{\frac{\alpha}{4} z^{2}} g\right)=\left(\partial_{z}+\frac{\alpha}{2} z\right) g .
$$

Thus it follows from equation (11.2) that the function $P_{1}^{\alpha}\left(\partial_{z}+\frac{\alpha}{2} z\right) \eta$ evolves by

$$
\partial_{\sigma}\left[P_{1}^{\alpha}\left(\partial_{z}+\frac{\alpha}{2} z\right) \eta\right]=-P_{1}^{\alpha}\left(L_{\alpha}+\alpha\right) P_{1}^{\alpha}\left(\partial_{z}+\frac{\alpha}{2} z\right) \eta+\sum_{k=2}^{6} D_{1,1}^{(k)}+D
$$

where $D_{1,1}^{(k)}$ is defined immediately after equation (11.26), and

$$
\begin{aligned}
D & :=-P_{1}^{\alpha}\left(\partial_{z}+\frac{\alpha}{2} z\right) V \eta \\
& =-P_{1}^{\alpha}\left[\partial_{z} V\right] \eta-P_{1}^{\alpha} V\left[\partial_{z}+\frac{\alpha}{2} z\right] \eta .
\end{aligned}
$$

The key observation here is that applying the operator $\partial_{z}+\frac{\alpha}{2} z$ gives an equation with an improved linear part.

Fix a ( $\tau$-scale) time $T$. Then Duhamel's principle lets us rewrite equation (11.61) as

$$
\begin{aligned}
P_{1}^{\alpha}\left(\partial_{z}+\frac{\alpha}{2} z\right) \eta(S)= & P_{1}^{\alpha} e^{-\left(L_{\alpha}+\alpha\right) S} P_{1}^{\alpha}\left(\partial_{z}+\frac{\alpha}{2} z\right) \eta(0) \\
& +\int_{0}^{S} P_{1}^{\alpha} e^{-\left(L_{\alpha}+\alpha\right)(S-\sigma)} P_{1}^{\alpha}\left[\sum_{n=2}^{6} D_{1,1}^{(n)}+D\right] d \sigma,
\end{aligned}
$$

where $P_{1}^{\alpha} e^{-\left(L_{\alpha}+\alpha\right) S} P_{1}^{\alpha}$ is defined in equation (11.15) and estimated in (11.18). For the remaining terms on the RHS, we derive the following estimates.

Lemma 11.7. If $A(\tau), B(\tau) \lesssim \beta^{-\frac{1}{20}}(\tau)$, then:

$$
\begin{gathered}
\left\|e^{\frac{\alpha}{4} z^{2}} D(\sigma)\right\|_{1,0} \lesssim \beta^{\frac{11}{10}}(\tau(\sigma))\left[M_{3,0}(T)+M_{2,1}(T)\right], \\
\left\|e^{\frac{\alpha z^{2}}{4}} \sum_{k=2,3} D_{1,1}^{(k)}(\sigma)\right\|_{1,0} \lesssim \beta^{\frac{6}{5}}(\tau(\sigma)) P(M(T), A(T)), \\
\left\|e^{\frac{\alpha}{4} z^{2}} D_{1,1}^{(4)}\right\|_{1,0} \lesssim\left\|e^{\frac{a y^{2}}{4}} N_{1}(a, b, \xi)\right\|_{1,1} \\
\lesssim \beta^{\frac{11}{10}}\left[M_{2,1}+M_{3,0}\right]+\beta^{\frac{6}{5}}\left(M_{\frac{11}{10}, 0}^{2}+M_{1,1} M_{\frac{11}{10}, 0}\right), \\
\left\|e^{\frac{\alpha}{4} z^{2}} D_{1,1}^{(5)}\right\|_{1,0} \lesssim\left\|e^{\frac{a y^{2}}{4}} N_{2}(a, b, \xi)\right\|_{1,1} \lesssim \beta^{\frac{6}{5}}(\tau), \\
\left\|e^{\frac{\alpha}{4} z^{2}} D_{1,1}^{(6)}\right\|_{1,0} \lesssim\left\|e^{\frac{a y^{2}}{4}} N_{3}(a, b, \xi)\right\|_{1,1} \lesssim \beta^{\frac{23}{10}}(\tau) .
\end{gathered}
$$

Proof. We first prove estimate (11.63). Direct computation leads to

$$
\begin{aligned}
\left|\langle z\rangle e^{\frac{\alpha}{4} z^{2}} D(\sigma)\right| & \lesssim \frac{b}{\left(1+b(\tau(\sigma)) z^{2}\right)^{2}}\left|e^{\frac{\alpha}{4} z^{2}} \eta\right|+\frac{1}{\langle z\rangle\left(1+b(\tau(\sigma)) z^{2}\right)}\left|\partial_{z} e^{\frac{\alpha}{4} z^{2}} \eta\right| \\
& \leq b^{-\frac{1}{2}}\langle z\rangle^{-3}\left|e^{\frac{\alpha}{4} z^{2}} \eta\right|+b^{-\frac{1}{2}}\langle z\rangle^{-2}\left|\partial_{z} e^{\frac{\alpha}{4} z^{2}} \eta\right| .
\end{aligned}
$$

To control the RHS, we apply inequality (11.7) to obtain the desired estimate. 
The proof of inequality (11.64) is very similar to the corresponding part of Lemma 11.3, hence is omitted.

The first inequalities in estimates (11.65)-(11.67) can be proved by the same method as estimate (11.34). So in what follows, we only prove the second ones.

Consider the second inequality in estimate (11.65) - an estimate which was not necessary in [9]. By direct computation, we obtain

$$
\begin{aligned}
\partial_{y} N_{1}(a, b, \xi)= & \frac{\partial_{y} v}{v^{2}} \frac{a+\frac{1}{2}}{2+b y^{2}}\left(e^{\frac{a}{4} y^{2}} \xi\right)^{2}+\frac{1}{v} \frac{2\left(a+\frac{1}{2}\right) b y}{\left(2+b y^{2}\right)^{2}}\left(e^{\left.\frac{a}{4} y^{2} \xi\right)^{2}}\right. \\
& -\frac{2}{v} \frac{a+\frac{1}{2}}{2+b y^{2}} e^{\frac{a}{4} y^{2}} \xi \partial_{y}\left(e^{\frac{a}{4} y^{2}} \xi\right) \\
= & : A_{1}+A_{2}+A_{3} .
\end{aligned}
$$

To bound $A_{1}$ and $A_{2}$, we note that $\left|v^{-1} \partial_{y} v\right| \lesssim \beta^{\frac{1}{2}}$ by (8.10), and use the consequences $\left|\frac{b y}{\sqrt{2+b y^{2}}}\right| \lesssim \beta^{\frac{1}{2}}$ and $|a| \leq 1$ of $(8.5)$ in Condition $[\mathrm{Cb}]$ to obtain

$$
\langle y\rangle^{-1}\left(\left|A_{1}\right|+\left|A_{2}\right|\right) \lesssim b^{\frac{1}{2}}\langle y\rangle^{-1} \frac{1}{1+b y^{2}}\left(e^{\frac{a}{4} y^{2}} \xi\right)^{2} \lesssim b^{\frac{1}{2}} b^{-\frac{3}{5}}\left(\langle y\rangle^{-\frac{11}{10}} e^{\frac{a}{4} y^{2}} \xi\right)^{2} .
$$

It follows that

$$
\left\|\langle y\rangle^{-1}\left(A_{1}+A_{2}\right)\right\|_{L^{\infty}} \lesssim b^{-\frac{1}{10}}\left\|\langle y\rangle^{-\frac{11}{10}} e^{\frac{a}{4} y^{2}} \xi\right\|_{\infty}^{2} \leq \beta^{\frac{6}{5}} M_{\frac{11}{10}, 0}^{2} .
$$

It is not hard to bound $A_{3}$ via the observation

$$
\begin{aligned}
\left\|\langle y\rangle^{-1} \frac{2}{v} \frac{a+\frac{1}{2}}{2+b y^{2}} e^{\frac{a}{4} y^{2}} \xi \partial_{y}\left(e^{\frac{a}{4} y^{2}} \xi\right)\right\|_{\infty} & \lesssim b^{-\frac{11}{20}}\left\|\langle y\rangle^{-\frac{11}{10}} e^{\frac{a}{4} y^{2}} \xi\right\|_{\infty}\left\|\langle y\rangle^{-1} \partial_{y}\left(e^{\frac{a}{4} y^{2}} \xi\right)\right\|_{\infty} \\
& \leq \beta^{\frac{6}{5}} M_{1,1} M_{\frac{11}{10}, 0} .
\end{aligned}
$$

When combined with estimate (11.68), this implies (11.65).

To derive the second inequality in estimate (11.66), we recall that $p=\partial_{y} v$ and $q=v^{-1} \partial_{\theta} v$ and then compute that

$\partial_{y}\left[e^{\frac{a}{4} y^{2}} N_{2}(a, b, \xi)\right]=\frac{2 p^{2}\left(p \partial_{y}^{2} v+q v^{-1} \partial_{y} \partial_{\theta} v-q^{2} v^{-1} \partial_{y} v\right)}{\left(1+p^{2}+q^{2}\right)} \partial_{y}^{2} v-\frac{p^{2} \partial_{y}^{3} v+2 p\left(\partial_{y}^{2} v\right)^{2}}{1+p^{2}+q^{2}}$.

Recall that we need to bound $\langle y\rangle^{-1} \partial_{y}\left[e^{\frac{a}{4} y^{2}} N_{2}(a, b, \xi)\right]$. To control $\langle y\rangle^{-1}\left|\partial_{y} v\right|$, we use Condition [Cb] for $M_{1,1}$, which implies that

$$
(1+|y|)^{-1}\left|\partial_{y} v\right| \lesssim 2 \beta
$$

To see this, we write $v=\sqrt{2+b y^{2} / a+\frac{1}{2}}+\phi$ and use the fact that

$$
\langle y\rangle^{-1}\left|\partial_{y} \phi\right| \leq \beta^{\frac{21}{20}} M_{1,1} \ll \beta .
$$

This observation, together with estimates (8.10)-(8.11) for $\left|\partial_{y}^{n} v\right|,(n=2,3)$, and $v^{-1}\left|\partial_{y} \partial_{\theta} v\right|$ yields the second inequality in estimate (11.66).

Now we turn to (11.67). As in equation (11.35), we decompose the new term $N_{3}$ into two parts, writing $N_{3}=N_{3,1}+N_{3,2}$. We will prove estimate (11.33) for $N_{3,1}$; the proof for $N_{3,2}$ follows readily from the decay estimates for $v^{-2} \partial_{\theta}^{2} v, v^{-1} \partial_{\theta} \partial_{y} v$, 
$v^{-2} \partial_{\theta} v$, and their $y$-derivatives provided by estimates (8.11) and (11.36). By direct computation, we have

$$
\partial_{y}\left[e^{\frac{a y^{2}}{4}} N_{3,1}\right]=-2 v^{-3} \partial_{y} v \partial_{\theta}^{2} v+\frac{1}{2}\left[\frac{2}{v^{2}}-1\right] \partial_{y} \partial_{\theta}^{2} v .
$$

For the first term on the RHS, we use estimates (8.10)-(8.11) to obtain

$$
v^{-3}\left|\partial_{y} v \partial_{\theta}^{2} v\right|=v^{-1}\left|\partial_{y} v\right| v^{-2}\left|\partial_{y} v \partial_{\theta}^{2} v\right| \lesssim \beta^{\frac{21}{20}}
$$

Estimating the second term on the RHS requires more work. We start by writing in the the more convenient form,

$$
\begin{aligned}
\langle y\rangle^{-1}\left|\left[\frac{2}{v^{2}}-1\right] \partial_{y} \partial_{\theta}^{2} v\right| & =\langle y\rangle^{-1}\left|\frac{1}{v}+1\right|\left|\frac{1}{v}-1\right|\left|\partial_{y} \partial_{\theta}^{2} v\right| \\
& \lesssim\langle y\rangle^{-1}\left|\frac{1}{v}-1\right|^{\frac{10}{11}}\left|\partial_{y} \partial_{\theta}^{2} v\right| \\
& =\left(v^{-2}\left|\partial_{y} \partial_{\theta}^{2} v\right|\right)^{\frac{5}{11}}\left|\partial_{y} \partial_{\theta}^{2} v\right|^{\frac{6}{11}}\left[\langle y\rangle^{-\frac{11}{10}}|v-1|\right]^{\frac{10}{11}}
\end{aligned}
$$

It follows from estimates (8.11) and (8.9) that the first two factors on the RHS here can be bounded by $\beta^{\frac{3}{4}} \epsilon_{0}^{\frac{6}{11}}$. To bound the final factor, we recall that $v=$ $\sqrt{2+b y^{2} / a+\frac{1}{2}}+e^{\frac{a}{4} y^{2}} \xi$. By definitions (8.2) and (8.3) for $M_{11 / 10,0}$ and $A$, respectively, we have

$$
\langle y\rangle^{-\frac{11}{10}}|v-1| \lesssim \beta^{\frac{1}{2}}+\beta^{\frac{1}{10}}\left[A+M_{11 / 10,0}\right] .
$$

Collecting the estimates above and using the consequences $A, M_{11 / 10,0} \lesssim \beta^{-\frac{1}{20}}$ of Condition [Cb], we obtain

$$
\langle y\rangle^{-1}\left|\left[\frac{2}{v^{2}}-1\right] \partial_{y} \partial_{\theta}^{2} v\right| \lesssim \beta^{\frac{53}{44}} \epsilon^{\frac{6}{11}} \leq \beta^{\frac{6}{5}}
$$

When combined with inequalities (11.70)-(11.71), this implies the desired estimate.

Finally, (11.67) follows easily from the estimates obtained earlier in the lemma; so we omit further details.

Now we return to equation (11.62). Applying the norm $\|\cdot\|_{1,0}=\left\|\langle y\rangle^{-1} \cdot\right\|_{\infty}$ to both sides yields

$$
\left\|e^{\frac{\alpha}{4} z^{2}} P_{1}^{\alpha}\left(\partial_{z}+\frac{\alpha}{2} z\right) \eta(S)\right\|_{1,0} \lesssim Y_{1}+Y_{2}+Y_{3}
$$

where

$$
\begin{aligned}
& Y_{1}:=e^{-\gamma S}\left\|e^{\frac{\alpha}{4} z^{2}} \eta(0)\right\|_{1,1}, \\
& Y_{2}:=\int_{0}^{S} e^{-\gamma(S-\sigma)} \sum_{k=2}^{6}\left\|e^{\frac{\alpha z^{2}}{4}} D_{1,1}^{(k)}(\sigma)\right\|_{1,0} \mathrm{~d} \sigma, \\
& Y_{3}:=\int_{0}^{S} e^{-\gamma(S-\sigma)}\left\|e^{\frac{\alpha z^{2}}{4}} D(\sigma)\right\|_{1,0} \mathrm{~d} \sigma .
\end{aligned}
$$

By inequalities (11.63)-(11.67) and the integral estimate (11.41), one has

$$
Y_{2} \lesssim\left[\int_{0}^{S} e^{-\gamma(S-\sigma)} \beta^{\frac{23}{20}} \mathrm{~d} \sigma\right] P(M(T), A(T)) \lesssim \beta^{\frac{23}{20}}(T) P(M(T), A(T)) .
$$


Similar reasoning yields

$$
Y_{3} \lesssim \beta^{\frac{11}{10}}(T)\left[M_{3,0}(T)+M_{2,1}(T)\right] .
$$

Estimate (11.7) and the slow decay of $\beta$ imply that

$$
Y_{1} \lesssim e^{-\gamma S}\|\phi(\cdot, 0)\|_{1,1} \lesssim \beta^{\frac{11}{10}}(T)(T) M_{1,1}(0) .
$$

Now collecting estimates (11.74)-(11.77), we obtain

$$
\begin{aligned}
& \beta^{-\frac{11}{10}}(T)\left\|e^{\frac{\alpha z^{2}}{4}} P_{1}^{\alpha}\left(\partial_{z}+\frac{\alpha}{2} z\right) \eta(S)\right\|_{1,0} \\
& \quad \lesssim M_{1,1}(0)+M_{3,0}(T)+M_{2,1}(T)+\beta^{\frac{1}{20}}(0) P(M(T), A(T)) .
\end{aligned}
$$

By equation (11.45), one has $\left.\| e^{\frac{\alpha z^{2}}{4}} P_{1}^{\alpha}\left(\partial_{z}+\frac{\alpha}{2} z\right) \eta(S)\right]\left\|_{1,0}=\right\| \phi(T) \|_{1,1}$. Thus by definition of $M_{1,1}$, one obtains

$$
M_{1,1}(T) \lesssim M_{1,1}(0)+M_{3,0}(T)+M_{2,1}(T)+\beta^{\frac{1}{20}}(0) P(M(T), A(T)) .
$$

Because $T$ was arbitrary, this proves estimate (9.15).

11.4. Proof of estimate (9.14). In this section, we prove estimate (9.14) for $M_{2,1}$ and thereby complete our proof of Proposition 9.2.

The same method used to derive equation (11.61) shows that $P_{2}^{\alpha}\left(\partial_{z}+\frac{\alpha}{2} z\right) \eta$ evolves by

$$
\partial_{\sigma}\left[P_{2}^{\alpha}\left(\partial_{z}+\frac{\alpha}{2} z\right) \eta\right]=-P_{2}^{\alpha}\left(\mathcal{L}_{\alpha}+\alpha\right) P_{2}^{\alpha}\left(\partial_{z}+\frac{\alpha}{2} z\right) \eta+\sum_{k=1}^{6} D_{2,1}^{(k)}+D_{7},
$$

where $D_{2,1}^{(k)},(k=1, \ldots, 6)$, are defined immediately after equation (11.26), and

$$
D_{7}:=-P_{2}^{\alpha} \eta \partial_{z} V .
$$

We fix a ( $\tau$-scale) time $T$ and apply Duhamel's principle to rewrite equation (11.78) in the form

$$
\begin{aligned}
P_{2}^{\alpha}\left(\partial_{z}+\frac{\alpha}{2} z\right) \eta(S)= & P_{2}^{\alpha} U_{2}(S, 0) e^{-\alpha S} P_{2}^{\alpha}\left(\partial_{z}+\frac{\alpha}{2} z\right) \eta(0) \\
& +\int_{0}^{S} P_{2}^{\alpha} U_{2}(S, \sigma) e^{-\alpha(S-\sigma)} P_{2}^{\alpha}\left[\sum_{n=1}^{6} D_{2,1}^{(n)}+D_{7}\right] \mathrm{d} \sigma
\end{aligned}
$$

where $U_{2}$ is defined in Proposition 11.2 and satisfies estimate (11.19). Terms on the RHS may be estimated as follows.

Lemma 11.8. If $A(\tau), B(\tau) \lesssim \beta^{-\frac{1}{20}}(\tau)$, then

$$
\left\|e^{\frac{\alpha}{4} z^{2}} D_{7}(\sigma)\right\|_{2,0} \lesssim \beta^{\frac{8}{5}}(\tau(\sigma)) M_{3,0}(T),
$$

and

$$
\left\|e^{\frac{\alpha z^{2}}{4}} \sum_{k=1}^{6} D_{2,1}^{(k)}(\sigma)\right\|_{2,0} \lesssim \beta^{\frac{33}{20}}(\tau(\sigma)) P(M(T), A(T))
$$


The proof is similar to but easier than that of Lemma 11.7, hence is omitted.

Continuing, we apply the $\|\cdot\|_{2,0}=\left\|\langle z\rangle^{-2} \cdot\right\|_{\infty}$ norm to equation (11.79), obtaining

$$
\left\|e^{\frac{\alpha}{4} z^{2}} P_{2}^{\alpha}\left(\partial_{z}+\frac{\alpha}{2} z\right) \eta(S)\right\|_{2,0} \lesssim Y_{1}+Y_{2}+Y_{3}
$$

where

$$
\begin{aligned}
Y_{1} & :=e^{-\gamma S}\left\|e^{\frac{\alpha}{4} z^{2}} \eta(0)\right\|_{2,1}, \\
Y_{2} & :=\int_{0}^{S} e^{-\gamma(S-\sigma)} \sum_{k=1}^{6}\left\|e^{\frac{\alpha z^{2}}{4}} D_{2,1}^{(k)}(\sigma)\right\|_{2,0} \mathrm{~d} \sigma, \\
Y_{3} & :=\int_{0}^{S} e^{-\gamma(S-\sigma)}\left\|e^{\frac{\alpha z^{2}}{4}} D_{7}(\sigma)\right\|_{2,0} \mathrm{~d} \sigma .
\end{aligned}
$$

By estimate (11.81) and the integral inequality (11.41), we have

$$
Y_{2} \lesssim\left[\int_{0}^{S} e^{-\gamma(S-\sigma)} \beta^{\frac{33}{20}} \mathrm{~d} \sigma\right] P(M(T), A(T)) \lesssim \beta^{\frac{33}{20}}(T) P(M(T), A(T)) .
$$

By similar reasoning, we get

$$
Y_{3} \lesssim \beta^{\frac{8}{5}}(T) M_{3,0}(T)
$$

Estimate (11.7) and the slow decay of $\beta$ imply that

$$
Y_{1} \lesssim e^{-\gamma S}\|\phi(\cdot, 0)\|_{2,1} \lesssim \beta^{\frac{8}{5}}(T)(T) M_{2,1}(0) .
$$

Collecting estimates (11.82)-(11.85), we conclude that

$$
\beta^{-\frac{8}{5}}(T)\left\|e^{\frac{\alpha z^{2}}{4}} P_{2}^{\alpha}\left(\partial_{z}+\frac{\alpha}{2} z\right) \eta(S)\right\|_{2,0} \lesssim M_{2,1}(0)+M_{3,0}(T)+\beta^{\frac{1}{20}}(0) P(M(T), A(T)) .
$$

Then we use equation (11.45) to rewrite the LHS, yielding

$$
\left.\| e^{\frac{\alpha z^{2}}{4}} P_{2}^{\alpha}\left(\partial_{z}+\frac{\alpha}{2} z\right) \eta(S)\right]\left\|_{2,0}=\right\| \phi(T) \|_{2,1} .
$$

Because $T$ was arbitrary, estimate (9.14) follows by recalling the definition of $M_{2,1}$.

This completes our proof of Proposition 9.2 and hence of Theorem 8.2.

\section{Proof of the Main Theorem}

The proof is almost identical to that in [9]. We provide a sketch below.

Proof of Main Theorem. For $b_{0}, c_{0}$ small enough, our Main Assumptions (see Section 2) imply that there exists $\tau_{1}>0$ such that the the necessary conditions including the hypotheses of Proposition 8.3 - for starting both bootstrap machines (see Sections 4.1 and 8.1) are satisfied for $\tau(t) \in\left[0, \tau_{1}\right]$. The outputs of both machines then show that the Main Assumptions hold at $\tau_{1}$, with smaller constants. So one can iterate this procedure to a larger interval $\left[0, \tau_{2}\right]$.

We now return to the original time scale $t$. The iteration above produces $t^{*} \leq T$, where $T \leq \infty$ denotes the maximal existence time of the solution, and a function $\lambda(t)$, admissible on $\left[0, t^{*}\right)$, such that Theorems $4.2,4.5,4.6,4.7$, and 8.2 hold on $\left[0, t^{*}\right)$. Observe that if the iteration cannot be continued past $t^{*}$, then either $t^{*}=T$ or $\lambda\left(t^{*}\right)=0$. Hence either (I) $t^{*}<T$ but $\lambda\left(t^{*}\right)=0$, or (II) $t^{*}=T=\infty$, or (III) $t^{*}=T<\infty$. 
We first claim that case (I) cannot occur. Indeed, if $\lambda\left(t^{*}\right)=0$, then it follows from Theorem 8.2 that

$$
0<u(0, \theta, t) \leq \lambda(t)\left[\sqrt{\frac{2}{1+C \beta(\tau(t))}}+C \beta^{\frac{8}{5}}(\tau(t))\right] \rightarrow 0
$$

as $t \nearrow t^{*}$, which implies that $T \leq t^{*}$.

We next show that case (II) cannot occur. Indeed, by Theorem 8.2, one has

$$
a(t)-\frac{1}{2}=-b(t)+\mathcal{O}\left(\beta^{2}(\tau(t))\right) \quad \text { and } \quad b(t)=\beta(\tau(t))\left[1+\mathcal{O}\left(\beta^{\frac{1}{2}}(\tau(t))\right)\right],
$$

where $\tau(t)=\int_{0}^{t} \lambda^{-2}(s) \mathrm{d} s$. So $\left|a(t)-\frac{1}{2}\right|=\mathcal{O}(\beta(\tau))$. To obtain a contradiction, suppose $T=\infty$. Then because $\partial_{t} \lambda=-a / \lambda<0$, we have $\tau \rightarrow \infty$ as $t \rightarrow \infty$, and hence $\left|a(t)-\frac{1}{2}\right| \rightarrow 0$. Because $\lambda^{2}(t)=\lambda_{0}^{2}-2 \int_{0}^{t} a(s) \mathrm{d} s$, it follows that there exists $\tilde{T}<\infty$ with $\lambda(T)=0$. Our proof for case (I) forces $T \leq \tilde{T}<\infty$, proving the claim.

Therefore, case (iii) is the only possibility. This means that there exists $\lambda(t)$, admissible on $[0, T)$ with $T<\infty$, such that Theorems 4.2, 4.5, 4.6, 4.7, and 8.2 hold for $t \in[0, T)$, with $\lambda(t) \searrow 0$ as $t \nearrow T$.

We establish the asymptotic behaviors of $\lambda(t), b(t)$, and $c(t):=\frac{1}{2}+a(t)$ as follows. Equation (12.1) shows that that $b(t) \rightarrow 0$ and $a(t) \rightarrow \frac{1}{2}$ as $t \nearrow T$. Hence as $t \nearrow T$, one has

$$
\lambda(t)=(1+o(1)) \sqrt{T-t} \text { and } \tau(t)=(1+o(1))(-\log (T-t)),
$$

as well as

$$
\beta(\tau(t))=(1+o(1))(-\log (T-t))^{-1} .
$$

This concludes the proof.

\section{Appendix A. Mean CURVATURe Flow of nORMal Graphs}

Here we derive the basic formulas that describe MCF of a normal graph over a cylinder. Although these equations are familiar to experts, we include the short derivation for the convenience of the reader, and to make the paper self-contained.

Write points in $\mathbb{R}^{n+2}$ as $(w, x)=\left(w^{1}, \ldots, w^{n+1}, x\right)$. The round cylinder $\mathbb{S}^{n} \times \mathbb{R}$ is naturally embedded as $\mathfrak{C}^{n+1}=\left\{(w, x):|w|^{2}=1\right\} \subset \mathbb{R}^{n+2}$. Let $\mathcal{M}_{t}$ denote a smooth family of smooth surfaces determined by $u: \mathcal{C}^{n+1} \times[0, T) \rightarrow \mathbb{R}_{+}$, namely

$$
\mathcal{M}_{t}=\left\{(u(w, x, t) w, x):(w, x) \in \mathfrak{C}^{n+1}, t \in[0, T)\right\} .
$$

For most of the derivation, we suppress time dependence. In what follows, we sum over repeated indices and restrict Roman indices to the range $1 \ldots n$.

For $(w, x) \in \mathbb{C}^{n+1}$, let $\left(e_{1}, \ldots, e_{n}, e_{n+1}=\frac{\partial}{\partial x}\right)$ denote an orthonormal basis of $T_{(w, x)} \mathrm{e}^{n+1} \subset T_{(w, x)} \mathbb{R}^{n+2}$, with $\left(e_{1}, \ldots, e_{n}\right)$ tangent to $\mathbb{S}^{n} \times\{x\}$. Let $D$ denote covariant differentiation on $\mathrm{e}^{n+1}$, and let $\nabla$ denote covariant differentiation on a round $\mathbb{S}^{n}$ of radius one. If

$$
F:(w, x) \mapsto(u(w, x) w, x),
$$

then, recalling that $1 \leq i \leq n$, one has

$$
\begin{aligned}
D_{i} F & =\left(\nabla_{i} u\right) w+u e_{i}, \\
D_{n+1} F & =\left(\partial_{x} u\right) w+e_{n+1} .
\end{aligned}
$$


It follows that the Riemannian metric $g$ induced on $\mathcal{M}$ has components

$$
\begin{aligned}
g_{i j} & =\left\langle D_{i} F, D_{j} F\right\rangle=u^{2} \delta_{i j}+\nabla_{i} u \nabla_{j} u, \\
g_{i n+1} & =\left\langle D_{i} F, D_{n+1} F\right\rangle=\partial_{x} u \nabla_{i} u, \\
g_{n+1 n+1} & =\left\langle D_{n+1} F, D_{n+1} F\right\rangle=1+\left(\partial_{x} u\right)^{2} .
\end{aligned}
$$

Note that in this Appendix (but nowhere else in this paper) the symbol $\langle\cdot, \cdot\rangle$ denotes the pointwise Euclidean inner product of $\mathbb{R}^{n+2}$.

An outward normal to $\mathcal{M}$ is

$$
N=u w-\nabla_{i} u e_{i}-u\left(\partial_{x} u\right) e_{n+1} .
$$

Noting that

$$
|N|^{2}=\left[1+\left(\partial_{x} u\right)^{2}\right] u^{2}+|\nabla u|^{2}
$$

we denote the outward unit normal by $\nu=|N|^{-1} N$. Straightforward calculations show that

$$
\begin{aligned}
|N| D_{j} \nu & =2\left(\nabla_{j} u\right) w+u e_{j}-\nabla_{j} \nabla u-\left(\partial_{x} u \nabla_{j} u+u \nabla_{j} \partial_{x} u\right) e_{n+1}+\{\cdots\} \nu, \\
|N| D_{n+1} \nu & =\left(\partial_{x} u\right) w-\nabla \partial_{x} u-\left[\left(\partial_{x} u\right)^{2}+u \partial_{x}^{2} u\right] e_{n+1}+\{\cdots\} \nu,
\end{aligned}
$$

where the terms in braces are easy to compute but irrelevant for what follows. One thus finds that the components of the second fundamental form $h$ are

$$
\begin{aligned}
h_{i j} & =\left\langle D_{i} F, D_{j} \nu\right\rangle=|N|^{-1}\left(g_{i j}+\nabla_{i} u \nabla_{j} u-u \nabla_{i} \nabla_{j} u\right), \\
h_{i n+1} & =\left\langle D_{i} F, D_{n+1} \nu\right\rangle=|N|^{-1}\left(g_{i n+1}-u \nabla_{i} \partial_{x} u\right), \\
h_{n+1 n+1} & =\left\langle D_{n+1} F, D_{n+1} \nu\right\rangle=|N|^{-1}\left(g_{n+1 n+1}-\left[\left(\partial_{x} u\right)^{2}+u \partial_{x}^{2} u\right]\right) .
\end{aligned}
$$

The standard trick to compute the mean curvature $H=\operatorname{tr}_{g}(h)$ of $\mathcal{M}$ is as follows. Fix any point of the graph and rotate the local orthonormal frame $\left(e_{1}, \ldots, e_{n}\right)$ so that all $\nabla_{i} u$ vanish except perhaps $\nabla_{n} u$. In these coordinates, one may identify $g$ and $h$ with block matrices

$$
g=\left(\begin{array}{cc}
u^{2} I_{n-1} & 0 \\
0 & P_{2}
\end{array}\right) \quad \text { and } \quad h=\frac{1}{|N|}\left(\begin{array}{cc}
u^{2} I_{n-1}-u \nabla \nabla u & 0 \\
0 & Q_{2}
\end{array}\right)
$$

respectively, where

$$
P_{2}=\left(\begin{array}{cc}
u^{2}+|\nabla u|^{2} & \partial_{x} u \nabla_{n} u \\
\partial_{x} u \nabla_{n} u & 1+\left(\partial_{x} u\right)^{2}
\end{array}\right)
$$

and

$$
Q_{2}=\left(\begin{array}{cc}
u^{2}+2|\nabla u|^{2}-u \nabla_{n} \nabla_{n} u & \partial_{x} u \nabla_{n} u-u \nabla_{n} \partial_{x} u \\
\partial_{x} u \nabla_{n} u-u \nabla_{n} \partial_{x} u & -u \partial_{x}^{2} u
\end{array}\right)
$$

Computing $\operatorname{tr}\left(g^{-1} h\right)$ and recasting the resulting terms in invariant notation, one obtains the coordinate-independent formula

$$
H=\frac{n|N|^{2}-u\left\{Q_{i j} \nabla_{i} \nabla_{j} u+\left(u^{2}+|\nabla u|^{2}\right) \partial_{x}^{2} u-2 \partial_{x} u\left\langle\nabla u, \nabla \partial_{x} u\right\rangle-u^{-1}|\nabla u|^{2}\right\}}{|N|^{3}},
$$

where $Q$ is the quasilinear elliptic operator on $\mathbb{S}^{n}$ defined below in equation (A.4).

Now we reintroduce time. It is a standard fact that MCF of $\mathcal{M}_{t}$ is equivalent (modulo time-dependent reparameterization by tangential diffeomorphisms) to the 
evolution equation

$$
\frac{u^{2}}{|N|^{2}} \partial_{t} u=-H\langle\nu, w\rangle=-\frac{H\langle N, w\rangle}{|N|}=-\frac{H u}{|N|},
$$

namely to $\partial_{t} u=-|N| H u^{-1}$. By equations (A.1) and (A.2), this is the quasilinear parabolic PDE

$$
\partial_{t} u=\frac{Q_{i j} \nabla_{i} \nabla_{j} u+\left(u^{2}+|\nabla u|^{2}\right) \partial_{x}^{2} u-2 \partial_{x} u\left\langle\nabla u, \nabla \partial_{x} u\right\rangle-u^{-1}|\nabla u|^{2}}{|\nabla u|^{2}+\left[1+\left(\partial_{x} u\right)^{2}\right] u^{2}}-\frac{n}{u},
$$

where $Q$ is the elliptic operator on $\mathbb{S}^{n}$ with coefficients

$$
Q_{i j}=\left[1+u^{-2}|\nabla u|^{2}+\left(\partial_{x} u\right)^{2}\right] \delta_{i j}-u^{-2} \nabla_{i} u \nabla_{j} u .
$$

\section{Appendix B. Interpolation estimates}

Here, we state and prove various interpolation inequalities used in this paper. We begin with an elementary embedding result.

Lemma B.1. Let $g:[0,2 \pi] \rightarrow \mathbb{R}$ be a smooth periodic function satisfying the condition $\int_{0}^{2 \pi} g(\theta) \mathrm{d} \theta=0$. Then for any $n \in \mathbb{Z}^{+}$, the following inequalities hold:

$$
\max _{\theta \in[0,2 \pi]}|g(\theta)| \leq \frac{5}{2}\left[\frac{1}{2 \pi} \int_{0}^{2 \pi}\left|\partial_{\theta}^{n} g\right|^{2} \mathrm{~d} \theta\right]^{\frac{1}{2}} \leq \frac{5}{2} \max _{\theta \in[0,2 \pi]}\left|\partial_{\theta}^{n} g\right| .
$$

Proof. We provide a detailed proof for $n=1$; the cases $n \geq 2$ are similar.

Recall that the Fourier decomposition $g(\theta)=\sum_{m=-\infty}^{\infty} a_{m} e^{i n \theta}$ and the Plancherel equality $\frac{1}{2 \pi} \int_{0}^{2 \pi}\left|\partial_{\theta} g\right|^{2} \mathrm{~d} \theta=\sum_{m=-\infty}^{\infty} m^{2}\left|a_{m}\right|^{2}$ hold for determined complex coefficients $a_{m}$. The key fact that $g \perp 1$ forces $a_{0}=0$. Using Hölder and weighted Cauchy-Schwarz, this fact allows us to obtain

$$
\max _{\theta \in[0,2 \pi]}|g(\theta)| \leq \sum_{m=-\infty}^{\infty}\left|a_{m}\right| \leq \frac{1}{2}\left(\varepsilon \sum_{m \neq 0}|m|^{-2}+\varepsilon^{-1} \sum_{m=-\infty}^{\infty} m^{2}\left|a_{m}\right|^{2}\right)
$$

for any $\varepsilon>0$. If $0<\varepsilon^{2}:=\sum_{m=-\infty}^{\infty} m^{2}\left|a_{m}\right|^{2}$, this becomes

$$
\max _{\theta \in[0,2 \pi]}|g(\theta)| \leq \frac{\varepsilon}{2}\left(\sum_{m \neq 0}|m|^{-2}+1\right)=\frac{\varepsilon}{2}\left(\frac{\pi^{2}}{3}+1\right)<\frac{5}{2} \varepsilon .
$$

The result follows.

The main result of this appendix is the following easy corollary.

Lemma B.2. Let $v$ satisfy Assumption [Cr], so that $\left|v_{2}\right| \leq \delta v_{1}$ with respect to the decomposition $v=v_{1}+v_{2}$ given in definition (4.2). Then for any $k \geq 0, m \geq 0$, and $n \geq 1$, one has

$$
v^{-k}\left|\partial_{y}^{m} \partial_{\theta}^{n} v\right| \lesssim\left[\frac{1}{2 \pi} \int_{0}^{2 \pi} v^{-2 k}\left|\partial_{y}^{m} \partial_{\theta}^{n+1} v\right|^{2} \mathrm{~d} \theta\right]^{\frac{1}{2}} \lesssim \max _{\theta \in[0,2 \pi]} v^{-k}\left|\partial_{y}^{m} \partial_{\theta}^{n+1} v\right| .
$$

Proof. By Lemma B.1, one has

$$
v_{1}^{-k}\left|\partial_{y}^{m} \partial_{\theta}^{n} v\right| \lesssim v_{1}^{-k}\left[\frac{1}{2 \pi} \int_{0}^{2 \pi}\left|\partial_{y}^{m} \partial_{\theta}^{n+1} v\right|^{2} \mathrm{~d} \theta\right]^{\frac{1}{2}} \leq v_{1}^{-k} \max _{\theta \in[0,2 \pi]}\left|\partial_{y}^{m} \partial_{\theta}^{n+1} v\right| .
$$

The result follows from this and the fact that $(1-\delta) v_{1} \leq v \leq(1+\delta) v_{1}$. 


\section{APPENDIX C. A PARABOLIC MAXIMUM PRINCIPLE FOR NONCOMPACT DOMAINS}

Parabolic maximum principles do not in general hold in noncompact domains without a growth restriction, even for the linear heat equation in Euclidean space. Here we extend Lemma 7.1 in [9], adding $\theta$-dependence and allowing exponential growth at infinity. This maximum principle is designed to apply to quantities $\left(v^{-k} \partial_{y}^{m} \partial_{\theta}^{n} v\right)^{\ell}$ in a time-dependent region $\left\{\beta(\tau) y^{2} \geq c\right\}$. (See Remark C.2 below.)

Proposition C.1. Given $T>0$ and a continuous function $b(\tau)$, define

$$
\Omega:=\left\{(y, \theta, \tau) \in \mathbb{R} \times \mathbb{S}^{1} \times(0, T]:|y| \geq b(\tau)\right\} .
$$

Let

$$
D:=\partial_{\tau}-\left(A_{1} \partial_{y}^{2}+A_{2} \partial_{\theta}^{2}+2 A_{3} \partial_{y} \partial_{\theta}+A_{4} \partial_{\theta}+A_{5} y \partial_{y}+B\right)
$$

be a differential operator with continuous coefficients in $\bar{\Omega}$.

(o) Suppose that $A_{1}, A_{5}$, and $B$ are uniformly bounded from above in $\Omega$, and the operator $D$ is parabolic in the sense that $A_{1} A_{2}>0$ and $A_{1} A_{2} \geq A_{3}^{2}$.

(I) Suppose that $u \in C^{2,1}(\Omega) \cap C^{0}(\bar{\Omega})$ is a D-subsolution, namely that

$$
\left(\partial_{\tau}-A_{1} \partial_{y}^{2}-A_{2} \partial_{\theta}^{2}-2 A_{3} \partial_{y} \partial_{\theta}-A_{4} \partial_{\theta}-A_{5} y \partial_{y}-B\right) u \leq 0 \quad \text { in } \quad \Omega .
$$

(II) Suppose that $u$ satisfies the parabolic boundary conditions

$$
u(y, \theta, 0) \leq 0 \text { if }|y| \geq b(0) ; \quad u(y, \theta, \tau) \leq 0 \text { if }|y|=b(\tau) \text { and } \tau \leq T .
$$

(III) Suppose that in $\Omega$,

$$
|u| \lesssim e^{\alpha y^{2}} \quad \text { for some } \alpha>0
$$

Then $u \leq 0$ in $\Omega$.

Proof. Because $B$ is bounded above, we may w.l.o.g. assume that $B \leq 0$. Otherwise, for $C>0$ sufficiently large, it suffices to prove the result for $\tilde{u}:=e^{-C \tau} u$, which satisfies $\left(\partial_{\tau}-A_{1} \partial_{y}^{2}-A_{2} \partial_{\theta}^{2}-2 A_{3} \partial_{y} \partial_{\theta}-A_{4} \partial_{\theta}-A_{5} y \partial_{y}-(B-C)\right) \tilde{u} \leq 0$.

For $0<\gamma<1 / 4$ to be chosen, define

$$
h(y, \tau):=\left(T_{0}-\tau\right)^{-1 / 2} e^{\gamma y^{2}\left(T_{0}-\tau\right)^{-1}},
$$

where $T_{0} \leq T$ is also to be chosen below, such that $0<T_{0}<\gamma / \alpha$. For $\varepsilon>0$, define

$$
w(y, \theta, \tau):=u(y, \theta, \tau)-\varepsilon[h(y, \tau)+\tau] .
$$

Let $T_{1}:=\frac{9}{10} T_{0}$. We claim that $w \leq 0$ for $\tau \in\left[0, T_{1}\right]$. To prove the claim, note that $w(\cdot, \cdot, 0) \leq-\varepsilon T_{0}^{-1 / 2}<0$ and $w(y, \theta, \tau) \rightarrow-\infty$ as $|y| \rightarrow \infty$, uniformly for $\tau \in\left[0, T_{1}\right]$. So if the claim is false, there is a first time $\tau \in\left(0, T_{1}\right]$ and a point $(y, \theta)$ with $|y|>b(\tau)$, where $0=w(y, \theta, \tau)=\max _{0 \leq \tau^{\prime} \leq \tau} w\left(\cdot, \cdot, \tau^{\prime}\right)$. At $(y, \theta, \tau)$, one has

$$
0 \leq \partial_{\tau} u-\varepsilon\left(\partial_{\tau} h+1\right) \leq-\varepsilon\left\{\left(\partial_{\tau}-A_{1} \partial_{y}^{2}-A_{5} y \partial_{y}\right) h-B(h+\tau)+1\right\} .
$$

But

$$
\left(\partial_{\tau}-A_{1} \partial_{y}^{2}-A_{5} y \partial_{y}\right) h=\frac{h}{2\left(T_{0}-\tau\right)}\left\{1-4 \gamma A_{1}+2 \gamma y^{2}\left[\frac{1-4 \gamma A_{1}}{T_{0}-\tau}-A_{5}\right]\right\} .
$$

By condition (o), one can choose $0<\gamma<\left(4 A_{1}\right)^{-1}$. Then choose $T_{0}$ small enough so that $A_{5} \leq\left(1-4 \gamma A_{1}\right) T_{0}^{-1}$. These choices ensure that $\left(\partial_{\tau}-A_{1} \partial_{y}^{2}-A_{5} y \partial_{y}\right) h \geq 0$, whence inequality (C.4) implies that $0 \leq-\varepsilon$, because we are assuming $B \leq 0$. This contradiction proves the claim.

Now letting $\varepsilon \searrow 0$ shows that $u \leq 0$ for $\tau \in\left[0, T_{1}\right]$. The theorem follows by repeating this argument on successive time intervals, $\left[T_{1}, \min \left\{2 T_{1}, T\right\}\right]$, etc. 
Remark C.2. In our applications, we only need to verify conditions (I)-(III), i.e. (C.1)-(C.3), in Proposition C.1, along with an upper bound for B. Indeed, parabolicity and an upper bound for $A_{1}$ follow easily from definition (2.8). Condition [Ca] bounds a from above. Condition [CO] implies upper bounds for $v^{-2}$. Hence condition (O) will always be satisfied by the operators in (2.5) and (3.4).

\section{ApPendix D. Estimates OF HIGHER-ORDER DERIVATIVES}

Recall that we defined $v_{m, n, k}$ in equation (3.1) and $E_{m, n, n, \ell}$ in definition (3.3). In this section, we estimate the nonlinear commutators ("error terms")

$$
E_{m, n}:=\int_{\mathbb{R}} \int_{\mathbb{S}^{1}}\left(\sum_{\ell=0}^{5} E_{m, n, n, \ell}\right) v_{m, n, n} \mathrm{~d} \theta \sigma \mathrm{d} y
$$

that appear in the evolution equations satisfied by the Lyapunov functionals $\Omega_{m, n}$ considered in Section 6 and defined in equation (6.1). We treat the cases that $3 \leq m+n \leq 5$ here, because the corresponding quantities were estimated for $m+n=2$ in the proofs of Lemmas 6.1-6.3 in Section 6.1. In the proofs in this Appendix, we use the fact that Assumptions [C0i]-[C1i] allow us to assume that estimates (4.4)-(4.6) of Theorem 4.2 hold globally.

Notation: For brevity, we suppress irrelevant coefficients in this section, writing $\simeq$ to denote an equality that holds up to computable and uniformly bounded constant coefficients. For example,

$$
(a+b)^{2} \simeq a^{2}+a b+b^{2} \lesssim a^{2}+b^{2} .
$$

Lemma D.1. If the first-order inequalities (4.4)-(4.6) and Conditions [C2]-[C3] hold, then there exist constants $0<C<\infty$ and $\rho=\rho(\Sigma)>0$, with $\rho(\Sigma) \searrow 0$ as $\Sigma \rightarrow \infty$, such that for all $m+n=3$ with $n \geq 1$, one has

$E_{m, n} \leq \rho \beta^{2}\left[\Omega_{m, n}^{\frac{1}{2}}+\beta \Omega_{4,0}^{\frac{1}{2}}+\Omega_{3,1}^{\frac{1}{2}}+\Omega_{2,2}^{\frac{1}{2}}+\Omega_{1,3}^{\frac{1}{2}}+\Omega_{0,4}^{\frac{1}{2}}\right]+C \beta^{\frac{3}{5}}\left(\Omega_{2,1}+\Omega_{1,2}+\Omega_{0,3}\right)$.

Proof. We provide a detailed proof for $E_{2,1}$.

It is easy to see that $\left|\left\langle E_{2,1,1,0}, v_{2,1,1}\right\rangle_{\sigma}\right| \lesssim \beta^{\frac{3}{5}} \Omega_{2,1}$. In the rest of the proof, we will frequently use estimates $(6.2)-(6.6)$, namely

$$
\left|\partial_{y} F_{\ell}\right| \lesssim \beta^{\frac{3}{5}}, \quad v^{-1}\left|\partial_{\theta} F_{\ell}\right| \lesssim \beta^{\frac{3}{2}}
$$

and

$$
\begin{aligned}
\left|\partial_{y}^{2} F_{\ell}\right| & \lesssim \beta^{\frac{6}{5}}+\left|\left(v_{3,0,0}\right)\right|+\left|\left(v_{2,1,1}\right)\right| \lesssim \beta, \\
v^{-1}\left|\partial_{y} \partial_{\theta} F_{\ell}\right| & \lesssim \beta^{2}+\left|\left(v_{2,1,1}\right)\right|+\left|\left(v_{1,2,2}\right)\right| \lesssim \beta^{\frac{3}{2}}, \\
v^{-2}\left|\partial_{\theta}^{2} F_{\ell}\right| & \lesssim \beta^{3}+\left|\left(v_{1,2,2}\right)\right|+\left|\left(v_{0,3,3}\right)\right| \lesssim \beta^{\frac{3}{2}},
\end{aligned}
$$

which hold under the hypotheses in place here. Direct computation establishes that

$$
\begin{aligned}
\left\langle E_{2,1,1,1}, v_{2,1,1}\right\rangle_{\sigma} \simeq & \left\langle v^{-1} \partial_{y}^{2} \partial_{\theta} F_{1},\left(v_{2,1,1}\right)\left(v_{2,0,0}\right)\right\rangle_{\sigma} \\
& +\left\langle\partial_{y}^{2} F_{1},\left(v_{2,1,1}\right)^{2}\right\rangle_{\sigma}+\left\langle v^{-1} \partial_{y} \partial_{\theta} F_{1},\left(v_{3,0,0}\right)\left(v_{2,1,1}\right)\right\rangle_{\sigma} \\
& +\left\langle\partial_{y} F_{1},\left(v_{3,1,1}\right)\left(v_{2,1,1}\right)\right\rangle_{\sigma}+\left\langle v^{-1} \partial_{\theta} F_{1},\left(v_{4,0,0}\right)\left(v_{2,1,1}\right)\right\rangle_{\sigma} \\
& +\left\langle F_{1},\left(v_{3,1,1}\right)\left(v_{2,1,1}\right)\left(v_{1,0,1}\right)+\left(v_{2,1,1}\right)^{2}\left[\left(v_{2,0,0}\right)+\left(v_{1,0,1}\right)^{2}\right]\right\rangle_{\sigma}
\end{aligned}
$$


Because we have stronger estimates for $\partial_{y} \partial_{\theta} F_{1}$ than we do for $\partial_{y}^{2} F_{1}$, we transform the first inner product above via integration by parts in $y$, yielding

$$
\begin{aligned}
\left\langle v^{-1} \partial_{y}^{2} \partial_{\theta} F_{1},\left(v_{2,1,1}\right)\left(v_{2,0,0}\right)\right\rangle_{\sigma} \simeq & \left\langle v^{-1} \partial_{y} \partial_{\theta} F_{1},\left(v_{3,1,1}\right)\left(v_{2,0,0}\right)+\left(v_{3,0,0}\right)\left(v_{2,1,1}\right)\right\rangle_{\sigma} \\
& +\left\langle v^{-1} \partial_{y} \partial_{\theta} F_{1},\left(v_{2,1,1}\right)\left(v_{2,0,0}\right)\left[\left(v_{1,0,1}\right)+\Sigma^{-\frac{1}{2}}\right]\right\rangle_{\sigma}
\end{aligned}
$$

where the final term comes from (6.7). Because the measure of $\left(\mathbb{S}^{1} \times \mathbb{R} ; \mathrm{d} \theta \sigma \mathrm{d} y\right)$ is finite, it then follows easily from the hypotheses of the lemma that

$$
\left|\left\langle E_{2,1,1,1}, v_{2,1,1}\right\rangle_{\sigma}\right| \leq \rho\left[\beta^{3} \Omega_{4,0}^{\frac{1}{2}}+\beta^{2} \Omega_{3,1}^{\frac{1}{2}}+\beta^{\frac{5}{2}} \Omega_{2,1}^{\frac{1}{2}}\right]+C \beta^{\frac{3}{5}}\left(\Omega_{2,1}+\Omega_{1,2}\right) .
$$

Integrating $\left\langle v^{-1} \partial_{y}^{2} \partial_{\theta} F_{2},\left(v_{2,1,1}\right)\left(v_{0,2,2}\right)\right\rangle_{\sigma}$ by parts in $\theta$, one obtains

$$
\begin{aligned}
\left\langle E_{2,1,1,2}, v_{2,1,1}\right\rangle_{\sigma} \simeq & \left\langle\partial_{y}^{2} F_{2},\left(v_{2,2,2}\right)\left(v_{0,2,2}\right)\right\rangle_{\sigma} \\
& +\left\langle\partial_{y}^{2} F_{2},\left(v_{2,1,1}\right)\left[\left(v_{0,3,3}\right)+\left(v_{0,2,2}\right)\left(v_{0,1,2}\right)\right]\right\rangle_{\sigma} \\
& +\left\langle v^{-1} \partial_{y} \partial_{\theta} F_{2},\left(v_{2,1,1}\right)\left[\left(v_{1,2,2}\right)+\left(v_{0,2,2}\right)\left(v_{1,0,1}\right)\right]\right\rangle_{\sigma} \\
& +\left\langle\partial_{y} F_{2},\left(v_{2,1,1}\right)\left[\left(v_{1,3,3}\right)+\left(v_{1,2,2}\right)\left(v_{0,1,2}\right)+\left(v_{0,3,3}\right)\left(v_{1,0,1}\right)\right]\right\rangle_{\sigma} \\
& +\left\langle\partial_{y} F_{2},\left(v_{2,1,1}\right)\left(v_{0,2,2}\right)\left[\left(v_{1,1,1}\right)+\left(v_{1,0,1}\right)\left(v_{0,1,2}\right)\right]\right\rangle_{\sigma} \\
& +\left\langle v^{-1} \partial_{\theta} F_{2},\left(v_{2,1,1}\right)\left[\left(v_{2,2,2}\right)+\left(v_{1,2,2}\right)\left(v_{1,0,1}\right)\right]\right\rangle_{\sigma} \\
& +\left\langle v^{-1} \partial_{\theta} F_{2},\left(v_{2,1,1}\right)\left(v_{0,2,2}\right)\left[\left(v_{2,0,0}\right)+\left(v_{1,0,1}\right)^{2}\right]\right\rangle_{\sigma} \\
& +\left\langle F_{2},\left(v_{2,1,1}\right)\left[\left(v_{1,3,3}\right)\left(v_{1,0,1}\right)+\left(v_{2,1,1}\right)\left(v_{0,1,2}\right)^{2}\right]\right\rangle_{\sigma} \\
& +\left\langle F_{2},\left(v_{2,1,1}\right)\left(v_{1,2,2}\right)\left[\left(v_{1,1,1}\right)+\left(v_{1,0,1}\right)\left(v_{0,1,2}\right)\right]\right\rangle_{\sigma} \\
& +\left\langle F_{2},\left(v_{2,1,1}\right)\left(v_{0,3,3}\right)\left[\left(v_{2,0,0}\right)+\left(v_{1,0,1}\right)^{2}\right]\right\rangle_{\sigma} \\
& +\left\langle F_{2},\left(v_{2,1,1}\right)\left(v_{2,0,0}\right)\left(v_{0,2,2}\right)\left(v_{0,1,2}\right)\right\rangle_{\sigma} \\
& +\left\langle F_{2},\left(v_{2,1,1}\right)\left(v_{0,2,2}\right)\left[\left(v_{1,1,1}\right)\left(v_{1,0,1}\right)+\left(v_{1,0,1}\right)^{2}\left(v_{0,1,2}\right)\right]\right\rangle_{\sigma},
\end{aligned}
$$

which shows that

$$
\left|\left\langle E_{2,1,1,2}, v_{2,1,1}\right\rangle_{\sigma}\right| \leq \rho\left[\beta^{\frac{5}{2}} \Omega_{2,2}^{\frac{1}{2}}+\beta^{2} \Omega_{1,3}^{\frac{1}{2}}+\beta^{\frac{7}{2}} \Omega_{2,1}^{\frac{1}{2}}\right]+C \beta\left(\Omega_{2,1}+\Omega_{1,2}+\Omega_{0,3}\right) .
$$

An similar computation, again integrating by parts in $\theta$, proves that

$$
\left|\left\langle E_{2,1,1,3}, v_{2,1,1}\right\rangle_{\sigma}\right| \leq \rho\left[\beta^{3} \Omega_{3,1}^{\frac{1}{2}}+\beta^{\frac{21}{10}} \Omega_{2,2}^{\frac{1}{2}}+\beta^{\frac{7}{2}} \Omega_{2,1}^{\frac{1}{2}}\right]+C \beta\left(\Omega_{2,1}+\Omega_{1,2}\right) .
$$

Integrating by parts in $\theta$ yet again, one calculates that

$$
\begin{aligned}
\left\langle E_{2,1,1,4}, v_{2,1,1}\right\rangle_{\sigma} & \left\langle\left\langle\partial_{y}^{2} F_{4},\left(v_{2,2,2}\right)\left(v_{0,1,2}\right)+\left(v_{2,1,1}\right)\left[\left(v_{0,2,2}\right)+\left(v_{0,1,2}\right)^{2}\right]\right\rangle_{\sigma}\right. \\
& +\left\langle v^{-1} \partial_{y} \partial_{\theta} F_{4},\left(v_{2,1,1}\right)\left[\left(v_{1,1,1}\right)+\left(v_{1,0,1}\right)\left(v_{0,1,2}\right)\right]\right\rangle_{\sigma} \\
& +\left\langle\partial_{y} F_{4},\left(v_{2,1,1}\right)\left[\left(v_{1,2,2}\right)+\left(v_{1,1,1}\right)\left(v_{1,0,1}\right)+\left(v_{0,2,2}\right)\left(v_{1,0,1}\right)\right]\right\rangle_{\sigma} \\
& +\left\langle v^{-1} \partial_{\theta} F_{4},\left(v_{2,1,1}\right)\left[\left(v_{2,1,1}\right)+\left(v_{2,0,0}\right)\left(v_{0,1,2}\right)\right]\right\rangle_{\sigma} \\
& +\left\langle v^{-1} \partial_{\theta} F_{4},\left(v_{2,1,1}\right)\left[\left(v_{1,1,1}\right)\left(v_{1,0,1}\right)+\left(v_{1,0,1}\right)^{2}\left(v_{0,1,2}\right)\right]\right\rangle_{\sigma} \\
& +\left\langle F_{4},\left(v_{2,1,1}\right)\left(v_{2,0,0}\right)\left[\left(v_{0,2,2}\right)+\left(v_{0,1,2}\right)^{2}\right]\right\rangle_{\sigma} \\
& +\left\langle F_{4},\left(v_{2,1,1}\right)\left(v_{1,1,1}\right)\left[\left(v_{1,1,1}\right)+\left(v_{1,0,1}\right)\left(v_{0,1,2}\right)\right]\right\rangle_{\sigma},
\end{aligned}
$$

yielding

$$
\left|\left\langle E_{2,1,1,4}, v_{2,1,1}\right\rangle_{\sigma}\right| \leq \rho \beta^{\frac{5}{2}}\left[\Omega_{2,2}^{\frac{1}{2}}+\Omega_{2,1}^{\frac{1}{2}}\right]+C \beta\left(\Omega_{2,1}+\Omega_{1,2}\right) .
$$


Finally, one computes

$$
E_{2,1,1,5} \simeq\left(v_{2,0,0}\right)\left(v_{0,1,2}\right)+\left(v_{1,1,1}\right)\left(v_{1,0,1}\right)+\left(v_{1,0,1}\right)^{2}\left(v_{0,1,2}\right)
$$

yielding

$$
\left|\left\langle E_{2,1,1,5}, v_{2,1,1}\right\rangle_{\sigma}\right| \leq \rho \beta^{2} \Omega_{2,1}^{\frac{1}{2}} .
$$

The estimate for $E_{2,1}$ follows when one combines the estimates above. (Note that in this case, the term $\Omega_{0,4}^{\frac{1}{2}}$ is not needed.)

The proof for $E_{1,2}$ is entirely analogous, except that it suffices here to integrate the leading term $\left\langle v^{-2} \partial_{y} \partial_{\theta}^{2} F_{1},\left(v_{1,2,2}\right)\left(v_{2,0,0}\right)\right\rangle_{\sigma}$ by parts in $\theta$. A typical subsequent term, also obtained by integrating by parts in $\theta$, is

$$
\begin{aligned}
\left\langle E_{1,2,2,2}, v_{1,2,2}\right\rangle_{\sigma} \simeq & \left\langle v^{-1} \partial_{y} \partial_{\theta} F_{2},\left(v_{1,3,3}\right)\left(v_{0,2,2}\right)\right\rangle_{\sigma} \\
& +\left\langle v^{-1} \partial_{y} \partial_{\theta} F_{2},\left(v_{1,2,2}\right)\left[\left(v_{0,3,3}\right)+\left(v_{0,2,2}\right)\left(v_{0,1,2}\right)\right]\right\rangle_{\sigma} \\
& +\left\langle v^{-2} \partial_{\theta}^{2} F_{2},\left(v_{1,2,2}\right)\left[\left(v_{1,2,2}\right)+\left(v_{0,2,2}\right)\left(v_{1,0,1}\right)\right]\right\rangle_{\sigma} \\
& +\left\langle\partial_{y} F_{2},\left(v_{1,2,2}\right)\left[\left(v_{0,4,4}\right)+\left(v_{0,3,3}\right)\left(v_{0,1,2}\right)\right]\right\rangle_{\sigma} \\
& +\left\langle\partial_{y} F_{2},\left(v_{1,2,2}\right)\left[\left(v_{0,2,2}\right)^{2}+\left(v_{0,2,2}\right)\left(v_{0,1,2}\right)^{2}\right]\right\rangle_{\sigma} \\
& +\left\langle v^{-1} \partial_{\theta} F_{2},\left(v_{1,2,2}\right)\left[\left(v_{1,3,3}\right)+\left(v_{1,2,2}\right)\left(v_{0,1,2}\right)+\left(v_{0,3,3}\right)\left(v_{1,0,1}\right)\right]\right\rangle_{\sigma} \\
& +\left\langle v^{-1} \partial_{\theta} F_{2},\left(v_{1,2,2}\right)\left(v_{0,2,2}\right)\left[\left(v_{1,1,1}\right)+\left(v_{1,0,1}\right)\left(v_{0,1,2}\right)\right]\right\rangle_{\sigma} \\
& +\left\langle F_{2},\left(v_{0,4,4}\right)\left(v_{1,2,2}\right)\left(v_{1,0,1}\right)+\left(v_{1,2,2}\right)^{2}\left[\left(v_{0,2,2}\right)+\left(v_{0,1,2}\right)^{2}\right]\right\rangle_{\sigma} \\
& +\left\langle F_{2},\left(v_{1,2,2}\right)\left(v_{0,3,3}\right)\left[\left(v_{1,1,1}\right)+\left(v_{1,0,1}\right)\left(v_{0,1,2}\right)\right]\right\rangle_{\sigma} \\
& +\left\langle F_{2},\left(v_{1,2,2}\right)\left(v_{0,2,2}\right)\left[\left(v_{1,1,1}\right)\left(v_{0,1,2}\right)+\left(v_{0,2,2}\right)\left(v_{1,0,1}\right)\right]\right\rangle_{\sigma} .
\end{aligned}
$$

The weakest estimate for $E_{1,2}$ again comes from its final term,

$$
\left|\left\langle E_{1,2,2,5}, v_{1,2,2}\right\rangle_{\sigma}\right| \leq \rho \beta^{2} \Omega_{1,2}^{\frac{1}{2}} .
$$

One estimates $E_{0,3}$ in exactly the same fashion. We omit further details.

Our final third-order quantity satisfies a slightly different estimate.

Lemma D.2. If the first-order inequalities (4.4)-(4.6) and Conditions [C2]-[C3] hold, then there exist constants $0<C<\infty$ and $\rho=\rho(\Sigma)>0$, with $\rho(\Sigma) \searrow 0$ as $\Sigma \rightarrow \infty$, such that

$$
E_{3,0} \leq \rho \beta^{\frac{3}{2}}\left[\Omega_{3,0}^{\frac{1}{2}}+\beta^{\frac{1}{10}} \Omega_{4,0}^{\frac{1}{2}}+\Omega_{3,1}^{\frac{1}{2}}+\Omega_{2,2}^{\frac{1}{2}}\right]+C \beta\left(\sum_{i+j=3} \Omega_{i, j}\right)+C \beta^{\frac{1}{2}} \Omega_{3,0}^{\frac{1}{2}} \Omega_{2,0}^{\frac{1}{2}} .
$$

Proof. Note that $E_{3,0,0,0}=0$. Integrating by parts in $y$, one calculates that

$$
\begin{aligned}
\left\langle E_{3,0,0,1},\left(v_{3,0,0}\right)\right\rangle_{\sigma} \simeq & \left\langle\partial_{y}^{2} F_{1},\left(v_{4,0,0}\right)\left(v_{2,0,0}\right)+\left(v_{3,0,0}\right)\left[\left(v_{3,0,0}\right)+\Sigma^{-\frac{1}{2}}\left(v_{2,0,0}\right)\right\rangle_{\sigma}\right. \\
& +\left\langle\partial_{y} F_{1},\left(v_{4,0,0}\right)\left(v_{3,0,0}\right)\right\rangle_{\sigma},
\end{aligned}
$$

where the $\Sigma^{-\frac{1}{2}}$ factor comes from (6.7). Thus,

$$
\left|\left\langle E_{3,0,0,1},\left(v_{3,0,0}\right)\right\rangle_{\sigma}\right| \leq \rho\left[\beta^{\frac{8}{5}} \Omega_{4,0}^{\frac{1}{2}}+\beta^{\frac{9}{5}} \Omega_{3,0}^{\frac{1}{2}}\right]+C \beta \Omega_{3,0} .
$$


Again integrating by parts in $y$, one estimates the quantity

$$
\begin{aligned}
\left\langle E_{3,0,0,3},\left(v_{3,0,0}\right)\right\rangle_{\sigma} \simeq & \left\langle\partial_{y}^{2} F_{3},\left(v_{4,0,0}\right)\left(v_{1,1,1}\right)\right\rangle_{\sigma} \\
& +\left\langle\partial_{y}^{2} F_{3},\left(v_{3,0,0}\right)\left[\left(v_{2,1,1}\right)+\left(v_{1,1,1}\right)\left(v_{1,0,1}\right)+\Sigma^{-\frac{1}{2}}\left(v_{1,1,1}\right)\right]\right\rangle_{\sigma} \\
& +\left\langle\partial_{y} F_{3},\left(v_{3,0,0}\right)\left[\left(v_{3,1,1}\right)+\left(v_{2,1,1}\right)\left(v_{1,0,1}\right)\right]\right\rangle_{\sigma} \\
& +\left\langle\partial_{y} F_{3},\left(v_{3,0,0}\right)\left[\left(v_{2,0,0}\right)+\left(v_{1,0,1}\right)^{2}\right]\left(v_{1,1,1}\right)\right\rangle_{\sigma} \\
& +\left\langle F_{3},\left(v_{3,1,1}\right)\left(v_{1,0,1}\right)+\left(v_{2,1,1}\right)\left[\left(v_{2,0,0}\right)+\left(v_{1,0,1}\right)^{2}\right]\right\rangle_{\sigma} \\
& +\left\langle F_{3},\left[\left(v_{3,0,0}\right)+\left(v_{2,0,0}\right)\left(v_{1,0,1}\right)+\left(v_{1,0,1}\right)^{3}\right]\left(v_{1,1,1}\right)\right\rangle_{\sigma}
\end{aligned}
$$

by

$$
\left|\left\langle E_{3,0,0,3},\left(v_{3,0,0}\right)\right\rangle_{\sigma}\right| \leq \rho\left[\beta^{\frac{5}{2}} \Omega_{4,0}^{\frac{1}{2}}+\beta^{\frac{3}{2}} \Omega_{3,1}^{\frac{1}{2}}+\beta^{\frac{13}{5}} \Omega_{3,0}^{\frac{1}{2}}\right]+C \beta\left(\Omega_{3,0}+\Omega_{2,1}\right) .
$$

The terms $\left\langle E_{3,0,0,2},\left(v_{3,0,0}\right)\right\rangle_{\sigma}$ and $\left\langle E_{3,0,0,4},\left(v_{3,0,0}\right)\right\rangle_{\sigma}$ have similar expansions and obey similar bounds; we omit the details. The critical final term,

$$
\left\langle E_{3,0,0,5},\left(v_{3,0,0}\right)\right\rangle_{\sigma} \simeq\left\langle\left(v_{2,0,0}\right)\left(v_{1,0,1}\right)+\left(v_{1,0,1}\right)^{3},\left(v_{3,0,0}\right)\right\rangle_{\sigma},
$$

is estimated by

$$
\left|\left\langle E_{3,0,0,5},\left(v_{3,0,0}\right)\right\rangle_{\sigma}\right| \leq C \beta^{\frac{1}{2}} \Omega_{3,0}^{\frac{1}{2}} \Omega_{2,0}^{\frac{1}{2}}+\rho \beta^{\frac{3}{2}} \Omega_{3,0}^{\frac{1}{2}} .
$$

(Note that we deal with the term $C \beta^{\frac{1}{2}} \Omega_{3,0}^{\frac{1}{2}} \Omega_{2,0}^{\frac{1}{2}}$ in the proof of Proposition 6.10.)

The next three lemmas prepare us to estimate $E_{m, n}$ for $4 \leq m+n \leq 5$.

Lemma D.3. If the first-order inequalities (4.4)-(4.6) and Conditions [C2]-[C3] hold, then one may estimate derivatives of $p:=\partial_{y} v$ by

$$
\begin{gathered}
\left|\partial_{y} p\right| \lesssim \beta^{\frac{3}{5}}, \quad v^{-1}\left|\partial_{\theta} p\right| \lesssim \beta^{\frac{3}{2}} \\
\left|\partial_{y}^{2} p\right| \lesssim \beta, \quad v^{-1}\left|\partial_{y} \partial_{\theta} p\right| \lesssim \beta^{\frac{3}{2}}, \quad v^{-2}\left|\partial_{\theta}^{2} p\right| \lesssim \beta^{\frac{3}{2}} \\
v^{-n}\left|\partial_{y}^{m} \partial_{\theta}^{n} p\right| \lesssim\left|\left(v_{m+1, n, n}\right)\right| \quad \text { for } \quad 3 \leq m+n \leq 4,
\end{gathered}
$$


and of $q:=v^{-1} \partial_{\theta} v$ by

$$
\begin{aligned}
v^{-n}\left|\partial_{y}^{m} \partial_{\theta}^{n} q\right| & \lesssim \beta^{\frac{3}{2}} \quad \text { for } \quad 1 \leq m+n \leq 2, \\
v^{-n}\left|\partial_{y}^{m} \partial_{\theta}^{n} q\right| & \lesssim\left|v_{m, n+1, n+1}\right|+\beta^{2} \quad \text { for } \quad m+n=3, \\
\left|\partial_{y}^{4} q\right| & \lesssim\left|\left(v_{4,1,1}\right)\right|+\beta^{\frac{3}{2}}\left|\left(v_{4,0,0}\right)\right|+\beta^{\frac{1}{2}}\left|\left(v_{3,1,1}\right)\right|+\beta^{\frac{21}{10}}, \\
v^{-1}\left|\partial_{y}^{3} \partial_{\theta} q\right| & \lesssim\left|\left(v_{3,2,2}\right)\right|+\beta^{\frac{3}{2}}\left|\left(v_{3,1,1}\right)\right|+\beta^{\frac{1}{2}}\left|\left(v_{2,2,2}\right)\right|+\beta^{\frac{21}{10}}, \\
v^{-2}\left|\partial_{y}^{2} \partial_{\theta}^{2} q\right| & \lesssim\left|\left(v_{2,3,3}\right)\right|+\beta^{\frac{3}{2}}\left|\left(v_{2,2,2}\right)\right|+\beta^{\frac{1}{2}}\left|\left(v_{1,3,3}\right)\right|+\beta^{\frac{21}{10}}, \\
v^{-3}\left|\partial_{y} \partial_{\theta}^{3} q\right| & \lesssim\left|\left(v_{1,4,4}\right)\right|+\beta^{\frac{3}{2}}\left|\left(v_{1,3,3}\right)\right|+\beta^{\frac{1}{2}}\left|\left(v_{0,4,4}\right)\right|+\beta^{3}, \\
v^{-4}\left|\partial_{\theta}^{4} q\right| & \lesssim\left|\left(v_{0,5,5}\right)\right|+\beta^{\frac{3}{2}}\left|\left(v_{0,4,4}\right)\right|+\beta^{3} .
\end{aligned}
$$

Proof. The estimates for $v^{-n}\left|\partial_{y}^{m} \partial_{\theta}^{n} p\right|$ are easy and are left to the reader.

The estimates for $v^{-n}\left|\partial_{y}^{m} \partial_{\theta}^{n} q\right|$ are obtained by computing and verifying all fourteen cases directly. For example, one has

$$
\begin{aligned}
v^{-2} \partial_{y}^{2} \partial_{\theta}^{2} q \simeq & \left(v_{2,3,3}\right)+\left(v_{2,2,2}\right)\left(v_{0,1,2}\right)+\left(v_{1,3,3}\right)\left(v_{1,0,1}\right) \\
& +\left(v_{2,1,1}\right)\left[\left(v_{0,2,2}\right)+\left(v_{0,1,2}\right)^{2}\right]+\left(v_{1,2,2}\right)\left[\left(v_{1,1,1}\right)+\left(v_{1,0,1}\right)\left(v_{0,1,2}\right)\right] \\
& +\left(v_{0,3,3}\right)\left[\left(v_{2,0,0}\right)+\left(v_{1,0,1}\right)^{2}\right]+\left(v_{2,0,0}\right)\left[\left(v_{0,2,2}\right)\left(v_{0,1,2}\right)+\left(v_{0,1,2}\right)^{3}\right] \\
& +\left(v_{1,1,1}\right)\left[\left(v_{1,1,1}\right)\left(v_{0,1,2}\right)+\left(v_{0,2,2}\right)\left(v_{1,0,1}\right)+\left(v_{1,0,1}\right)\left(v_{0,1,2}\right)^{2}\right] \\
& +\left(v_{1,0,1}\right)^{2}\left(v_{0,1,2}\right)^{3} .
\end{aligned}
$$

The remaining calculations are similar and unenlightening, hence omitted.

Our next results extend estimates (6.2)-(6.6) to higher derivatives $v^{-n}\left|\partial_{y}^{m} \partial_{\theta}^{n} F_{\ell}\right|$.

Lemma D.4. If the first-order inequalities (4.4)-(4.6) and Conditions [C2]-[C3] hold, then third derivatives of the coefficients $F_{\ell}$ introduced in definition (2.8) may be estimated by

$$
\left|\partial_{y}^{3} F_{\ell}\right| \lesssim\left|\left(v_{4,0,0}\right)\right|+\left|\left(v_{3,1,1}\right)\right|+\beta^{\frac{8}{5}},
$$

and for $m+n=3$ with $n \geq 1$, by

$$
v^{-n}\left|\partial_{y}^{m} \partial_{\theta}^{n} F_{\ell}\right| \lesssim\left|\left(v_{m+1, n, n}\right)\right|+\left|\left(v_{m, n+1, n+1}\right)\right|+\beta^{2} .
$$

Proof. After a wee bit of calculus, one computes, for example, that

$$
\left|\partial_{y}^{3} F_{\ell}\right| \lesssim\left(\left|\partial_{y} p\right|+\left|\partial_{y} q\right|\right)^{3}+\left(\left|\partial_{y} p\right|+\left|\partial_{y} q\right|\right)\left(\left|\partial_{y}^{2} p\right|+\left|\partial_{y}^{2} q\right|\right)+\left|\partial_{y}^{3} p\right|+\left|\partial_{y}^{3} q\right|
$$

and

$$
\begin{aligned}
\left|\partial_{y}^{2} \partial_{\theta} F_{\ell}\right| \lesssim & \left(\left|\partial_{y} p\right|+\left|\partial_{y} q\right|\right)^{2}\left(\left|\partial_{\theta} p\right|+\left|\partial_{\theta} q\right|\right)+\left(\left|\partial_{y} p\right|+\left|\partial_{y} q\right|\right)\left(\left|\partial_{y} \partial_{\theta} p\right|+\left|\partial_{y} \partial_{\theta} q\right|\right) \\
& +\left(\left|\partial_{\theta} p\right|+\left|\partial_{\theta} q\right|\right)\left(\left|\partial_{y}^{2} p\right|+\left|\partial_{y}^{2} q\right|\right)+\left|\partial_{y}^{2} \partial_{\theta} p\right|+\left|\partial_{y}^{2} \partial_{\theta} q\right|
\end{aligned}
$$

and then applies Lemma D.3. The remaining inequalities are derived similarly. 
Lemma D.5. If the first-order inequalities (4.4)-(4.6) and Conditions [C2]-[C3] hold, then fourth derivatives of the coefficients $F_{\ell}$ introduced in definition (2.8) may be estimated by

$$
\begin{gathered}
\left|\partial_{y}^{4} F_{\ell}\right| \lesssim\left|\left(v_{5,0,0}\right)\right|+\left|\left(v_{4,1,1}\right)\right|+\beta^{\frac{3}{5}}\left|\left(v_{4,0,0}\right)\right|+\beta^{\frac{1}{2}}\left|\left(v_{3,1,1}\right)\right|+\beta^{2}, \\
v^{-1}\left|\partial_{y}^{3} \partial_{\theta} F_{\ell}\right| \lesssim\left|\left(v_{4,1,1}\right)\right|+\left|\left(v_{3,2,2}\right)\right|+\beta^{\frac{3}{2}}\left|\left(v_{4,0,0}\right)\right|+\beta^{\frac{3}{5}}\left|\left(v_{3,1,1}\right)\right|+\beta^{\frac{1}{2}}\left|\left(v_{2,2,2}\right)\right|+\beta^{\frac{21}{10}}, \\
v^{-2}\left|\partial_{y}^{2} \partial_{\theta}^{2} F_{\ell}\right| \lesssim\left|\left(v_{3,2,2}\right)\right|+\left|\left(v_{2,3,3}\right)\right|+\beta^{\frac{3}{2}}\left|\left(v_{3,1,1}\right)\right|+\beta^{\frac{3}{5}}\left|\left(v_{2,2,2}\right)\right|+\beta^{\frac{1}{2}}\left|\left(v_{1,3,3}\right)\right|+\beta^{\frac{21}{10}}, \\
v^{-3}\left|\partial_{y} \partial_{\theta}^{3} F_{\ell}\right| \lesssim\left|\left(v_{2,3,3}\right)\right|+\left|\left(v_{1,4,4}\right)\right|+\beta^{\frac{3}{2}}\left|\left(v_{2,2,2}\right)\right|+\beta^{\frac{3}{5}}\left|\left(v_{1,3,3}\right)\right|+\beta^{\frac{1}{2}}\left|\left(v_{4,0,0}\right)\right|+\beta^{\frac{13}{5}}, \\
v^{-4}\left|\partial_{\theta}^{4} F_{\ell}\right| \lesssim\left|\left(v_{1,4,4}\right)\right|+\left|\left(v_{0,5,5}\right)\right|+\beta^{\frac{3}{2}}\left(\left|\left(v_{1,3,3}\right)\right|+\left|\left(v_{0,4,4}\right)\right|\right)+\beta^{3} .
\end{gathered}
$$

Proof. One proceeds as in Lemma D.4, calculating, for example, that

$$
\begin{aligned}
\left|\partial_{y}^{3} \partial_{\theta} F_{\ell}\right| \lesssim & \left|\partial_{y}^{3} \partial_{\theta} p\right|+\left|\partial_{y}^{3} \partial_{\theta} q\right| \\
& +\left(\left|\partial_{y}^{3} p\right|+\left|\partial_{y}^{3} q\right|\right)\left(\left|\partial_{\theta} p\right|+\left|\partial_{\theta} q\right|\right) \\
& +\left(\left|\partial_{y}^{2} \partial_{\theta} p\right|+\left|\partial_{y}^{2} \partial_{\theta} q\right|\right)\left(\left|\partial_{y} p\right|+\left|\partial_{y} q\right|\right) \\
& +\left(\left|\partial_{y} \partial_{\theta} p\right|+\left|\partial_{y} \partial_{\theta} q\right|\right)\left(\left|\partial_{y}^{2} p\right|+\left|\partial_{y}^{2} q\right|+\left|\partial_{y} p\right|^{2}+\left|\partial_{y} q\right|^{2}\right) \\
& +\left(\left|\partial_{y}^{2} p\right|+\left|\partial_{y}^{2} q\right|\right)\left(\left|\partial_{y} p\right|\left|\partial_{\theta} p\right|+\left|\partial_{y} q\right|\left|\partial_{\theta} q\right|\right) \\
& +\left(\left|\partial_{y} p\right|+\left|\partial_{y} q\right|\right)^{3}\left(\left|\partial_{\theta} p\right|+\left|\partial_{\theta} q\right|\right),
\end{aligned}
$$

and then applying Lemma D.3. The other estimates are obtained in like fashion.

We are now prepared to bound the "error terms" $E_{m, n}$ of orders four and five. As indicated in Section 6.3, our work below is considerably simplified because these estimates do not need to be sharp, and because they can use Proposition 6.10.

Lemma D.6. If the first-order inequalities (4.4)-(4.6), Conditions [C2]-[C3], and $L_{\sigma}^{2}$ inequalities (6.9) hold, then there exists $0<C<\infty$ such that for all $m+n=4$, one has

$$
E_{m, n} \leq C \beta^{r}\left(\sum_{4 \leq i+j \leq 5} \Omega_{i, j}^{\frac{1}{2}}\right)+C \beta^{\frac{1}{2}}\left(\sum_{4 \leq i+j \leq 5} \Omega_{i, j}\right),
$$

where $r=\frac{8}{5}$ if $n=0$ and $r=2$ otherwise.

Proof. It is easy to see that $\left|\left\langle E_{m, n, n, 0}, v_{m, n, n}\right\rangle_{\sigma}\right| \lesssim \beta^{\frac{3}{5}} \Omega_{m, n}$.

In the remainder of the proof, we write $D^{k} F_{\ell}$ to denote any sum of terms $v^{-j} \partial_{y}^{i} \partial_{\theta}^{j} F_{\ell}$ with $i+j=k$; and we write $D^{k} v$ to denote any sum of terms $v_{i, j, j}$ of total weight $i+j=k$. For example, $D^{2} v \simeq\left(v_{2,0,0}\right)+\left(v_{1,1,1}\right)+\left(v_{0,2,2}\right)$.

For $\ell=1,2,3$, carefully adapting the proof of Lemma D.1 to the case that $m+n=4$ shows that after integration by parts, ${ }^{10}$ one obtains an estimate of the

\footnotetext{
${ }^{10}$ In contrast to the proof of Lemma D.1, the choice of variable with which to integrate does not matter here unless $n=0$, because our estimates do not need to be sharp.
} 
following form,

$$
\begin{aligned}
\left|\left\langle E_{m, n, n, \ell}, v_{m, n, n}\right\rangle_{\sigma}\right| \lesssim & \left\langle\left|D^{3} F_{\ell}\right|,\left|D^{5} v\right|\left|D^{2} v\right|+\left|v_{m, n, n}\right|\left(\left|D^{3} v\right|+\Sigma^{-\frac{1}{2}}\right)\right\rangle_{\sigma} \\
& +\left\langle\left|D^{3} F_{\ell}\right|,\left|v_{m, n, n}\right|\left(\left|D^{3} v\right|+\left|D^{2} v\right|\left|D^{1} v\right|\right)\right\rangle_{\sigma} \\
& +\left\langle\left|D^{2} F_{\ell}\right|,\left|v_{m, n, n}\right|\left(\left|D^{4} v\right|+\left|D^{3} v\right|\left|D^{1} v\right|+\left|D^{2} v\right|^{2}\right)\right\rangle_{\sigma} \\
& +\left\langle\left|D^{1} F_{\ell}\right|,\left|v_{m, n, n}\right|\left(\left|D^{5} v\right|+\left|D^{4} v\right|\left|D^{1} v\right|+\left|D^{3} v\right|\left|D^{2} v\right|\right)\right\rangle_{\sigma} \\
& +\left\langle\left|v_{m, n, n}\right|,\left|D^{5} v\right|\left|D^{1} v\right|+\left|D^{4} v\right|\left|D^{2} v\right|+\left|D^{3} v\right|^{2}\right\rangle_{\sigma} .
\end{aligned}
$$

Examination of the terms above using our hypotheses and Lemma D.4 shows that any terms which are quadratic in derivatives of orders four or five are multiplied by factors whose decay is at least of order $\beta^{\frac{1}{2}}$. Any derivatives of orders four or five that do not occur in quadratic combinations appear with factors whose decay is at least of order $\beta^{r}$, where $r=\frac{8}{5}$ if $n=0$ and $r=2$ otherwise. A similar but simpler estimate holds for $\left|\left\langle E_{m, n, n, 4}, v_{m, n, n}\right\rangle_{\sigma}\right|$.

For the critical final term, one uses estimate (6.9) to see that

$$
\begin{aligned}
\left|\left\langle E_{m, n, n, 5}, v_{m, n, n}\right\rangle_{\sigma}\right| & \simeq\left\langle\left|v_{m, n, n}\right|,\left|D^{3} v\right|\left|D^{1} v\right|+\left|D^{2} v\right|\left(\left|D^{2} v\right|+\left|D^{1} v\right|^{2}\right)+\left|D^{1} v\right|^{4}\right\rangle_{\sigma} \\
& \lesssim C \beta^{r} \Omega_{m, n}^{\frac{1}{2}} .
\end{aligned}
$$

The result follows.

Lemma D.7. If the first-order inequalities (4.4)-(4.6), Conditions [C2]-[C3], and $L_{\sigma}^{2}$ inequalities (6.9) hold, then there exists $0<C<\infty$ such that for all $m+n=5$, one has

$$
E_{m, n} \leq C \beta^{\frac{21}{10}}\left(\sum_{5 \leq i+j \leq 6} \Omega_{i, j}^{\frac{1}{2}}\right)+C \beta^{\frac{1}{2}}\left(\sum_{4 \leq i+j \leq 6} \Omega_{i, j}\right) .
$$

Proof. It is clear that $\left|\left\langle E_{m, n, n, 0}, v_{m, n, n}\right\rangle_{\sigma}\right| \lesssim \beta^{\frac{3}{5}} \Omega_{m, n}$.

As in the proof of Lemma D.6, we write $D^{k} F_{\ell}$ to denote any sum of terms $v^{-j} \partial_{y}^{i} \partial_{\theta}^{j} F_{\ell}$ with $i+j=k$; and we write $D^{k} v$ to denote any sum of terms $v_{i, j, j}$ of total weight $i+j=k$.

For $\ell=1,2,3$, adapting the proof of Lemma D.1 to the case that $m+n=5$ shows that before integration by parts, one has

$$
\begin{aligned}
\left|\left\langle E_{m, n, n, \ell}, v_{m, n, n}\right\rangle_{\sigma}\right| \lesssim & \left\langle\left|D^{5} F_{\ell}\right|,\left|v_{m, n, n}\right|\left|D^{2} v\right|\right\rangle_{\sigma} \\
& +\left\langle\left|D^{4} F_{\ell}\right|,\left|v_{m, n, n}\right|\left(\left|D^{3} v\right|+\left|D^{2} v\right|\left|D^{1} v\right|\right)\right\rangle_{\sigma} \\
& +\left\langle\left|D^{3} F_{\ell}\right|,\left|v_{m, n, n}\right|\left(\left|D^{4} v\right|+\left|D^{3} v\right|\left|D^{1} v\right|+\left|D^{2} v\right|^{2}\right)\right\rangle_{\sigma} \\
& +\left\langle\left|D^{2} F_{\ell}\right|,\left|v_{m, n, n}\right|\left(\left|D^{5} v\right|+\left|D^{4} v\right|\left|D^{1} v\right|+\left|D^{3} v\right|\left|D^{2} v\right|\right)\right\rangle_{\sigma} \\
& +\left\langle\left|D^{1} F_{\ell}\right|,\left|v_{m, n, n}\right|\left(\left|D^{6} v\right|+\left|D^{5} v\right|\left|D^{1} v\right|+\left|D^{4} v\right|\left|D^{2} v\right|+\left|D^{3} v\right|^{2}\right)\right\rangle_{\sigma} \\
& +\left\langle\left|v_{m, n, n}\right|,\left(\left|D^{6} v\right|\left|D^{1} v\right|+\left|D^{5} v\right|\left|D^{2} v\right|+\left|D^{4} v\right|\left|D^{3} v\right|\right)\right\rangle_{\sigma} .
\end{aligned}
$$

Integrating by parts proves that the first inner product on the RHS above may be bounded by

$$
\left\langle\left|D^{5} F_{\ell}\right|,\left|v_{m, n, n}\right|\left|D^{2} v\right|\right\rangle_{\sigma} \lesssim\left\langle\left|D^{4} F_{\ell}\right|,\left|D^{6} v\right|\left|D^{2} v\right|+\left|v_{m, n, n}\right|\left(\left|D^{3} v\right|+\Sigma^{-\frac{1}{2}}\right)\right\rangle_{\sigma} .
$$


Integrating by parts is also necessary to show that the third inner product on the RHS above may be bounded by

$$
\begin{aligned}
\left\langle\left|D^{3} F_{\ell}\right|,\right. & \left.\left|v_{m, n, n}\right|\left(\left|D^{4} v\right|+\left|D^{3} v\right|\left|D^{1} v\right|+\left|D^{2} v\right|^{2}\right)\right\rangle_{\sigma} \\
\lesssim & \left\langle\left|D^{2} F_{\ell}\right|,\left|D^{6} v\right|\left(\left|D^{4} v\right|+\left|D^{3} v\right|\left|D^{1} v\right|+\left|D^{2} v\right|^{2}\right)\right\rangle_{\sigma} \\
& +\left\langle\left|D^{2} F_{\ell}\right|,\left|v_{m, n, n}\right|\left(\left|D^{5} v\right|+\left|D^{4} v\right|\left|D^{1} v\right|+\left|D^{3} v\right|\left|D^{2} v\right|\right)\right\rangle_{\sigma} \\
& +\left\langle\left|D^{2} F_{\ell}\right|,\left|v_{m, n, n}\right|\left(\left|D^{4} v\right|+\left|D^{3} v\right|\left|D^{1} v\right|+\left|D^{2} v\right|^{2}\right) \Sigma^{-\frac{1}{2}}\right\rangle_{\sigma} .
\end{aligned}
$$

Examination of all the terms above using our hypotheses and Lemmas D.4-D.5 proves that any terms that are quadratic in derivatives of orders four, five, or six are multiplied by factors whose decay is at least of order $\beta^{\frac{1}{2}}$. Any derivatives of orders five or six not occuring in quadratic combinations appear with factors whose decay is at least of order $\beta^{\frac{11}{5}}$. (Derivatives of order four only occur in quadratic combinations.) A similar but simpler estimate holds for $\left|\left\langle E_{m, n, n, 4}, v_{m, n, n}\right\rangle_{\sigma}\right|$.

For the critical final term, one uses estimate (6.9) to see that

$$
\begin{aligned}
\left|\left\langle E_{m, n, n, 5}, v_{m, n, n}\right\rangle_{\sigma}\right| \simeq & \left\langle\left|v_{m, n, n}\right|,\left|D^{4} v\right|\left|D^{1} v\right|+\left|D^{3} v\right|\left(\left|D^{2} v\right|+\left|D^{1} v\right|^{2}\right)\right\rangle_{\sigma} \\
& +\left\langle\left|v_{m, n, n}\right|,\left|D^{2} v\right|\left(\left|D^{2} v\right|\left|D^{1} v\right|+\left|D^{1} v\right|^{3}\right)+\left|D^{1} v\right|^{5}\right\rangle_{\sigma} \\
\lesssim & C \beta^{\frac{21}{10}} \Omega_{m, n}^{\frac{1}{2}}+C \beta^{\frac{1}{2}}\left(\sum_{4 \leq i+j \leq 5} \Omega_{i, j}\right)
\end{aligned}
$$

The result follows.

\section{REFERENCES}

[1] Angenent, Sigurd B.; Isenberg, James; Knopf, Dan. Formal matched asymptotics for degenerate Ricci flow neckpinches. Nonlinearity 24 (2011), 2265-2280.

[2] Angenent, Sigurd B.; Knopf, Dan. Precise asymptotics of the Ricci flow neckpinch. Comm. Anal. Geom. 15 (2007), no. 4, 773-844.

[3] Angenent, Sigurd B.; Velázquez, J. J. L. Degenerate neckpinches in mean curvature flow. J. Reine Angew. Math. 482 (1997), 15-66.

[4] Brakke, Kenneth A. The motion of a surface by its mean curvature. Mathematical Notes, 20. Princeton University Press, Princeton, N.J., 1978.

[5] Chen, Yun Gang; Giga, Yoshikazu; Goto, Shun'ichi. Uniqueness and existence of viscosity solutions of generalized mean curvature flow equations. J. Differential Geom. 33 (1991), no. 3, 749-786.

[6] Colding, Tobias Holck; Minicozzi, William P., II. Generic mean curvature flow I; generic singularities. Ann. of Math. (2) 175 (2012), no. 2, 755-833.

[7] Dejak, Steven; Gang, Zhou; Sigal, Israel Michael; Wang, Shuangcai. Blow-up in nonlinear heat equations. Adv. in Appl. Math. 40 (2008), no. 4, 433-481.

[8] Evans, Lawrence C.; Spruck, Joel. Motion of level sets by mean curvature. I. J. Differential Geom. 33 (1991), no. 3, 635-681.

[9] Gang, Zhou; Sigal, Israel Michael. Neck pinching dynamics under mean curvature flow. J. Geom. Anal. 19 (2009), no. 1, 36-80.

[10] Huisken, Gerhard. Asymptotic behavior for singularities of the mean curvature flow. J. Differential Geom. 31 (1990), no. 1, 285-299.

[11] Huisken, Gerhard; Sinestrari, Carlo Mean curvature flow with surgeries of two-convex hypersurfaces. Invent. Math. 175 (2009), no. 1, 137-221.

[12] Simon, Miles Mean curvature flow of rotationally symmetric hypersurfaces. Honours thesis, Australian National University, 1990. 
(Zhou Gang) California Institute of Technology

E-mail address: gzhou@caltech.edu

(Dan Knopf) University of Texas at Austin

E-mail address: danknopf@math.utexas.edu

URL: http://www.ma.utexas.edu/users/danknopf/

(Israel Michael Sigal) University of Toronto

E-mail address: im.sigal@utoronto.ca

URL: http://www.math.toronto.edu/sigal/ 